

\title{
Berry Curvature and Topology in Low-Dimensional Crystals
}

\author{
Raffaele Battilomo
}


PhD thesis, Utrecht University, June 2021

DOI: $10.33540 / 731$

Printed by: ProefschriftMaken.nl

ISBN: 978-94-6423-286-8

About the cover: Perhaps some very complicated band structure. Probably not. Made with Blender. 


\title{
Berry Curvature and Topology in Low-Dimensional Crystals
}

\author{
Berry-Kromming en Topologie in \\ Laag-Dimensionale Kristallen
}

(met een samenvatting in het Nederlands)

\section{Proefschrift}

ter verkrijging van de graad van doctor aan de Universiteit Utrecht op gezag van de rector magnificus, prof. dr. H.R.B.M. Kummeling, ingevolge het besluit van het college voor promoties in het openbaar te verdedigen op woensdag 23 juni 2021 des middags te 12.15 uur

door

\section{Raffaele Battilomo}

geboren op 21 juni 1992

te Rome, Italië 
Promotor:

Prof. dr. ir. H.T.C. Stoof

Copromotor:

Dr. C. Ortix 



\section{Contents}

Preface v v

List of publications vii

1 Introduction 1

1.1 Berry Phase . . . . . . . . . . . . . . . . 1

1.1.1 Berry Phase in a Two-Level System . . . . . . . . . 3

1.1.2 Berry Phase in a Two-Dimensional Semiconductor Ring 5

1.1.3 Berry Phase in Crystals . . . . . . . . . . . . . 9 9

1.2 Anomalous Velocity . . . . . . . . . . . . . . . . 10

1.3 Non-Linear Hall Effect Induced by Berry Curvature Dipole . 13

1.4 Topological Insulators . . . . . . . . . . . . . . . 15

1.5 Outline .......................... 18

2 Berry Curvature Dipole in Strained Graphene: a Fermi Surface $\begin{array}{ll}\text { Warping Effect } & 21\end{array}$

2.1 Berry Curvature Dipole in Strained Monolayer Graphene . . 23

2.2 Berry Curvature Dipole in Strained Bilayer Graphene . . . . 25

2.3 Conclusions . . . . . . . . . . . . . . . . . 30

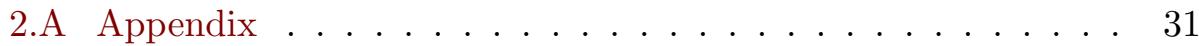

2.A.1 Monolayer Graphene . . . . . . . . . . . . . . . 31

2.A.2 Bilayer Graphene . . . . . . . . . . . . . . . 32

\section{Anomalous Planar Hall Effect in Two-Dimensional Trigonal Crys-} $\begin{array}{ll}\text { tals } & 37\end{array}$

3.1 Anomalous Planar Hall Effect . . . . . . . . . . . . . . . 40

3.2 Symmetry analysis . . . . . . . . . . . . . . . 42

3.3 Model . . . . . . . . . . . . . . . . . . . . . 44

3.4 Conclusions . . . . . . . . . . . . . . . . . . . . 48

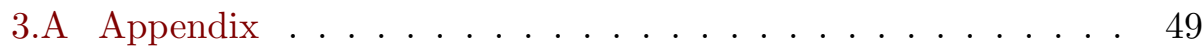

3.A.1 Linear Planar Hall Effects in 2D and 3D . . . . . . . 49 
Contents

3.A.2 Phase Behavior of the Model Hamiltonian . . . . . . . 50

3.A.3 Semiclassical Linear Planar Hall Effect . . . . . . . . . 51

3.A.4 Semiclassical Non-Linear Planar Hall Effect . . . . . . 52

4 Anomalous Planar Hall Effect in a Rashba Two-Dimensional Electron Gas

4.1 Model Hamiltonian . . . . . . . . . . . . . . . . . . . . . 53

4.2 Anomalous Planar Hall Effect . . . . . . . . . . . . . . . . 58

5 Spin Field-Effect Transistor in a Quantum Spin-Hall Device $\quad 61$

5.1 In Gap States of the BHZ Hamiltonian . . . . . . . . . . . . 62

5.2 Effective 1D Model . . . . . . . . . . . . . . . . . 67

5.2 .1 1D Hamiltonian . . . . . . . . . . . . . . 67

5.2.2 Scattering Matrix Approach . . . . . . . . . . . . . 68

5.2 .3 Spin-Polarized Conductance . . . . . . . . . . . . . 72

5.3 2D Tight-Binding Model . . . . . . . . . . . . 75

5.4 Conclusions . . . . . . . . . . . . . . . . 77

6 Tuning topology in thin films of topological insulators by strain $\begin{array}{ll}\text { gradients } & \mathbf{7 9}\end{array}$

6.1 Strained TI thin films . . . . . . . . . . . . . . 80

6.2 Strain gradient-induced topological phase transitions . . . . . 82

6.3 Narrow band-gap materials . . . . . . . . . . . . . 86

6.4 Conclusions . . . . . . . . . . . . . . . . . . 89

6.A Appendix ........................... 90

6.A.1 Derivation of the inhomogeneous strain $\mathbf{k} \cdot \mathbf{p}$ Hamiltonian ..................... 90

6.A.2 $\mathrm{Sb}_{2} \mathrm{Te}_{3}$ Thin films . . . . . . . . . . . . . 92

6.A.3 Supplemental Figures . . . . . . . . . . . . 93

7 Conclusions $\quad 95$

7.1 Summary and Results . . . . . . . . . . . . . . . . 95

$\begin{array}{ll}\text { Bibliography } & 97\end{array}$

$\begin{array}{ll}\text { English Summary } & 109\end{array}$

$\begin{array}{ll}\text { Dutch Summary } & 111\end{array}$ 
Contents

Acknowledgments 113

About the author 



\section{Preface}

"Stilicidi casus lapidem cavat."

- Lucretius

This thesis is the result of four years of research, done at the Institute for Theoretical Physics of Utrecht University under the supervision of dr. Carmine Ortix. 



\section{List of publications}

This thesis is based on the following publications:

$\diamond$ Chapter 2 is based on R. Battilomo, N. Scopigno and C. Ortix, Phys. Rev. Lett. 123, 196403 (2019)

$\diamond$ Chapter 3 is based on R. Battilomo, N. Scopigno and C. Ortix, Phys. Rev. Research 3, L012006 (2021)

$\diamond$ Chapter 5 is based on R. Battilomo, N. Scopigno and C. Ortix, Phys. Rev. B 98, 075147 (2018)

$\diamond$ Chapter 6 is based on R. Battilomo, N. Scopigno and C. Ortix, Phys. Rev. B 100, 115131 (2019) 



\section{Introduction}

This thesis relies on two crucial concepts that for the past four decades have been the subject of intense research in condensed matter physics: the electronic Berry curvature effects in crystals [1] and topological insulators [2]. These concepts are of course deeply connected, making their first combined appearance in the description of the integer quantum Hall effect. Since then, a new way of classifying phases of matter based on band topology has emerged and a vast class of Berry curvature induced effects have been observed in condensed matter systems.

Chapters 2, 3 and 4 are deeply reliant on the concept of anomalous velocity induced by the Berry curvature of the Bloch bands. Whereas, in Chapters 5 and 6 we discuss, respectively, the transport properties of a quantum spin Hall disk and the effect of inhomogeneous strain gradients in threedimensional topological insulators. The following introductory sections are the basic building blocks to fully appreciate the subjects discussed in the coming chapters.

\subsection{Berry Phase}

The concept of Berry phase, introduced by Michael Berry [3], is of fundamental importance in understanding a vast class of material properties $[1,4]$, such as orbital magnetism, electronic polarization and different types of Hall effects. However, the original paper where the Berry phase first made its appearance had no apparent connection to condensed matter systems: it described the adiabatic evolution of an eigenstate when the external parameters it depends upon are looped in parameter space. Take for instance a set of time-dependent parameters $\mathbf{R}(t)=\left(R_{1}(t), R_{2}(t), \ldots\right)$ and a Hamiltonian $\mathcal{H}(\mathbf{R}(t))$. We can study how the eigenstates of $\mathcal{H}$ change when the parameters $\mathbf{R}(t)$ evolve adiabatically in a path $\mathcal{C}$ in parameter space. This can be done by introducing an instantaneous eigenbasis $|n(\mathbf{R})\rangle$ for each value of 


\section{Introduction}

$\mathbf{R}(t)$ such that,

$$
\mathcal{H}(\mathbf{R}(\mathbf{t}))|n(\mathbf{R}(\mathbf{t}))\rangle=\varepsilon(\mathbf{R}(\mathbf{t}))|n(\mathbf{R}(\mathbf{t}))\rangle
$$

By making use of the quantum adiabatic theorem, a system which finds itself in an eigenstate $|n(\mathbf{R}(0))\rangle$ will stay in an instantaneous eigenstate $|n(\mathbf{R}(t))\rangle$ during the adiabatic evolution. Hence the state $|\psi(t)\rangle$ at time $t$ differs from $|n(\mathbf{R}(t))\rangle$ only in the phase picked up during the adiabatic process,

$$
\left|\psi_{n}(t)\right\rangle=e^{i \gamma_{n}(t)} e^{-i \int_{0}^{t} d t^{\prime} \varepsilon_{n}\left(\mathbf{R}\left(t^{\prime}\right)\right)}|n(\mathbf{R}(t))\rangle
$$

The second exponent is the known dynamical phase factor which depends upon the time-evolution of the parameter dependent eigenenergy. By inserting Eq. 1.2 into the time-dependent Schroedinger equation and multiplying both sides by $\langle n(\mathbf{R})|$,

$$
\left\langle n(\mathbf{R}(t))\left|i \hbar \partial_{t}\right| \psi_{n}(t)\right\rangle=\left\langle n(\mathbf{R}(t))|\mathcal{H}(\mathbf{R}(t))| \psi_{n}(t)\right\rangle
$$

we can determine the phase factor $\gamma_{n}(t)$ which is given by the line integral in parameter space,

$$
\gamma_{n}=\int_{\mathcal{C}} d \mathbf{R} \cdot \mathcal{A}_{n}(\mathbf{R}) .
$$

On top of the dynamical phase, the state at time $t$ picks up an additional phase $\gamma_{n}$ during the adiabatic evolution. $\mathcal{A}_{n}(\mathbf{R})$ is known as the Berry connection and is directly related to the eigenstates of $\mathcal{H}$,

$$
\mathcal{A}_{n}(\mathbf{R})=i\left\langle n(\mathbf{R})\left|\partial_{\mathbf{R}}\right| n(\mathbf{R})\right\rangle
$$

In general $\gamma_{n}$ is gauge dependent as can be easily seen by performing a smooth gauge transformation to the instantaneous eigenstates,

$$
|n(\mathbf{R})\rangle \rightarrow e^{i \zeta(\mathbf{R})}|n(\mathbf{R})\rangle
$$

This transforms the Berry connection,

$$
\mathcal{A}_{n}(\mathbf{R}) \rightarrow \mathcal{A}_{n}(\mathbf{R})-\partial_{\mathbf{R}} \zeta(\mathbf{R})
$$


leading to an overall variation in $\gamma_{n}$ given by $\zeta(\mathbf{R}(0))-\zeta(\mathbf{R}(T))$. Typically one can always choose $\zeta(\mathbf{R})$ such that $\gamma_{n}$ vanishes, as was already noticed back in 1928 by Fock [5]. What was discovered only decades later was that when the adiabatic evolution is cyclic, i.e. $\mathbf{R}(0)=\mathbf{R}(T)$, since the the gauge transformation $\zeta(\mathbf{R})$ is required to be single-valued, one must have,

$$
\zeta(\mathbf{R}(0))-\zeta(\mathbf{R}(T))=2 \pi n \quad n \in \mathbb{Z} .
$$

This proves that when considering the adiabatic evolution of $\mathcal{H}(\mathbf{R})$ in a loop in parameter space, the Berry phase $\gamma_{n}=\oint_{\mathcal{C}} d \mathbf{R} \cdot \mathcal{A}_{n}(\mathbf{R})$ is a gauge-invariant, hence physical, quantity that only depends on the geometry of the closed path $\mathcal{C}$.

In the following we will consider the parameter space to be at most threedimensional. By applying Stoke's Theorem we can recast Eq. 1.4,

$$
\gamma_{n}=\int_{\mathcal{C}} d \mathbf{R} \cdot \mathcal{A}_{n}(\mathbf{R})=\int_{\mathcal{S}} d \mathbf{S} \cdot \boldsymbol{\Omega}_{n}(\mathbf{R}) .
$$

where $\boldsymbol{\Omega}_{n}(\mathbf{R})=\nabla_{\mathbf{R}} \times \mathcal{A}_{n}(\mathbf{R})$ is the gauge-invariant Berry curvature which can be intuitively thought as a magnetic field in parameter space. The Berry curvature is a local quantity encoding the geometric properties of the wavefunctions that, as we will show, also has far reaching consequences in the description of electronic dynamics in crystals [1].

The above derivation of the Berry phase and curvature relies on the absence of degeneracies in the energy spectrum during the adiabatic cycle. In the case of degeneracies being present a non-Abelian description has to be employed [6].

\subsubsection{Berry Phase in a Two-Level System}

It is particularly useful to study a two-level system since it uncovers various important properties of the Berry phase and Berry curvature and how they are related to a particular topological invariant. The Hamiltonian of a twolevel system takes the form,

$$
\mathcal{H}=\mathbf{h}(\mathbf{R}) \cdot \boldsymbol{\sigma}
$$




\section{Introduction}

where $\boldsymbol{\sigma}$ is a vector of Pauli matrices. We can employ spherical coordinates and parametrize the vector $\mathbf{h}=h(\sin \theta \cos \phi, \sin \theta \sin \phi, \cos \theta)$. The eigenstates with energies $\pm h$ are then given by,

$$
\left|u_{-}\right\rangle=\left(\begin{array}{c}
\sin \frac{\theta}{2} e^{i \phi} \\
-\cos \frac{\theta}{2}
\end{array}\right), \quad\left|u_{+}\right\rangle=\left(\begin{array}{c}
\cos \frac{\theta}{2} e^{-i \phi} \\
\sin \frac{\theta}{2}
\end{array}\right) .
$$

Let us now consider the lowest energy level $\left|u_{-}\right\rangle$which for convenience we will denote as $|u\rangle$. From Eq. 1.5 the Berry connection is given by,

$$
\begin{aligned}
& \mathcal{A}_{\theta}=\left\langle u \mid i \partial_{\theta} u\right\rangle=0 \\
& \mathcal{A}_{\phi}=\left\langle u \mid i \partial_{\phi} u\right\rangle=\sin ^{2} \frac{\theta}{2}
\end{aligned}
$$

and the Berry curvature reads,

$$
\Omega_{\theta \phi}=\partial_{\theta} \mathcal{A}_{\phi}-\partial_{\phi} \mathcal{A}_{\theta}=\frac{1}{2} \sin \theta .
$$

The phase of $\left|u_{-}\right\rangle$is not well defined at the south pole where $\theta=\pi$. In this point the first component of $\left|u_{-}\right\rangle$is $e^{i \phi}$ despite the fact that it cannot be defined since both $x$ and $y$ coordinates are zero. We may choose a different gauge and multiply $\left|u_{-}\right\rangle$by $e^{-i \phi}$ to make it smooth. But once again $\left|u_{-}\right\rangle$ will be well defined everywhere except for the north pole, at $\theta=0$. Under this gauge $\mathcal{A}_{\theta}=0$ and $\mathcal{A}_{\phi}=-\cos ^{2} \frac{\theta}{2}$ while the Berry curvature remains unchanged. This is not unexpected since, as explained in the previous section, the Berry curvature is a gauge-invariant quantity whereas the Berry connection is not. If $\mathbf{h}(\mathbf{R})$ depends on a set of parameters $\mathbf{R}$ we have,

$$
\Omega_{R_{i}, R_{j}}=\frac{1}{2} \frac{\partial(\phi, \cos \theta)}{\partial\left(R_{i}, R_{j}\right)}
$$

where $\partial(\phi, \cos \theta) / \partial\left(R_{i}, R_{j}\right)$ is the jacobian of the transformation from $\mathbf{R}$ to $(\theta, \phi)$. By considering the case in which $\mathbf{h}=(x, y, z)$, since $\Omega_{i}=\epsilon_{i j k} \Omega_{R_{i}, R_{j}}$, we can write the Berry curvature in its vector form,

$$
\boldsymbol{\Omega}=\frac{1}{2} \frac{\mathbf{h}}{h^{3}} .
$$


Eq. 1.16 is the field generated by a monopole of strength $1 / 2$ (in parameter space) at the degeneracy point $\mathbf{h}=0$. For the other energy level the monopole will have opposite strength, $-1 / 2$. We have found that degeneracy points in parameter space act as sources and drains of the Berry curvature. Integrating the Berry curvature over a sphere containing the monopole yields,

$$
\int_{\mathcal{S}^{2}} d \theta d \phi \Omega_{\theta \phi}=2 \pi .
$$

Note that when integrating on $\mathcal{S}^{2}$ the Hamiltonian is always gapped since the degeneracy point is enclosed in the sphere. The integral of the Berry curvature over a closed manifold is quantized in units of $2 \pi$ and is equal to the number of net monopoles inside. This number is a topological invariant called the Chern number, responsible for a number of quantization effects, the prime example being the integer quantum Hall effect which we will discuss later on.

\subsubsection{Berry Phase in a Two-Dimensional Semiconductor Ring}

As discussed above the Berry phase is a gauge-invariant, and hence physical, quantity. It follows that it should be possible to observe it by exploiting the wave-like nature of electronic quantum states such as electrons. In this section we discuss how the Berry phase of the electronic spin can be measured in a particular interferometric setup comprised of a quasi one-dimensional semiconducting ring $[7,8]$. The setup described here shares several common features with the one we adopt in Chapter 5 where we study the transport properties of a quantum spin-Hall disk.

The system we are interested in studying is a two-dimensional semiconducting ring with Rashba spin-orbit coupling $[9,10]$. The Rashba interaction is a particular type of spin-orbit which appears in non-centrosymmetric materials and can be typically tuned by means of an external electric field $\mathbf{E}$. This kind of spin-orbit coupling locks the electronic spin and momentum degrees of freedom together and is an essential tool in the field of spinorbitronics. As we will now show, it also plays a major role in the Berry phase acquired by electrons in a semiconducting ring. By sending the width of the ring to zero an effective one-dimensional Hamiltonian can be derived 


\section{Introduction}

[11]. In polar coordinates it is given by,

$\mathcal{H}_{1 \mathrm{D}}=-\frac{\hbar \omega_{0}}{2} \partial_{\phi}^{2}+\frac{\hbar \omega_{R}}{2}\left(\cos \phi \sigma_{x}+\sin \phi \sigma_{y}\right)\left(-i \partial_{\phi}\right)-i \frac{\hbar \omega_{R}}{4}\left(\cos \phi \sigma_{y}-\sin \phi \sigma_{x}\right)$

where we defined the frequencies $\omega_{0}=\frac{\hbar}{m^{*} r_{0}^{2}}$ and $\omega_{R}=\frac{2 \alpha_{R}}{\hbar r_{0}}$ with $m^{*}$ the effective electronic mass, $r_{0}$ the ring radius and $\alpha_{R}$ the Rashba spin-orbit coupling constant. The last term in Eq. 1.18 is essential to ensure that $\mathcal{H}$ is hermitian. By using the following ansatz for the eigenfunctions of the system,

$$
\Psi_{n}^{s}=e^{i n \phi}\left(\begin{array}{c}
e^{-i \frac{\phi}{2}} \chi_{1}^{s} \\
e^{i \frac{\phi}{2}} \chi_{2}^{s}
\end{array}\right)
$$

where $n$ is a positive or negative half-integer number eigenvalue of the angular momentum operator $L_{\phi}=i \partial_{\phi}$. The eigenvalues of the system are then given by,

$$
\left(\begin{array}{cc}
\frac{\hbar \omega_{0}}{2}\left(n-\frac{1}{2}\right)^{2}-\epsilon & \frac{\hbar \omega_{R}}{2}\left(n+\frac{1}{2}\right)-\frac{\hbar \omega_{R}}{4} \\
\frac{\hbar \omega_{R}}{2}\left(n-\frac{1}{2}\right)+\frac{\hbar \omega_{R}}{4} & \frac{\hbar \omega_{0}}{2}\left(n+\frac{1}{2}\right)^{2}-\epsilon
\end{array}\right)
$$

leading to the quantized energies,

$$
\epsilon_{n}^{s}=\frac{\hbar \omega_{0}}{2}\left(n^{2}+\frac{1}{4}+s n \sqrt{1+Q_{r}^{2}}\right) .
$$

Here $Q_{r}=\frac{\omega_{R}}{\omega_{0}}$ is the adiabatic parameter and $s= \pm 1$ represents the sign of the spin projection along a certain quantization axis that changes with each value of the angle $\phi$. Indeed, the Rashba coupling can be thought of as an effective radial magnetic field $B_{S O} \propto \mathbf{E} \times \mathbf{p}$, with $\mathbf{p}$ the electron's momentum and $\mathbf{E}$ the electric field caused by the structural inversion asymmetry, to which the spins tend to align (see Fig. 1.2a). At zero Rashba $s$ is exactly the eigenvalue of the Pauli matrix $\sigma_{z}$ labelling up and down spin states. The energies in Eq. 1.21 are given by two shifted parabolas (Fig. 1.1b), signature of the Rashba spin-orbit coupling. At each allowed energy we find four states, two having a positive band velocity $\left(v_{s}=\frac{d \epsilon_{n}^{s}}{d n}\right)$ and opposite $s$, moving clockwise along the ring while the other two are the time-reversed pair with opposite spin, travelling counter-clockwise. 
(a)

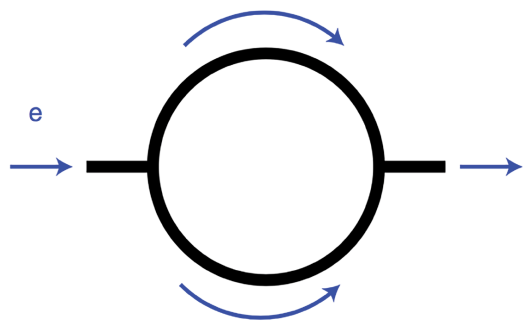

$(b)$

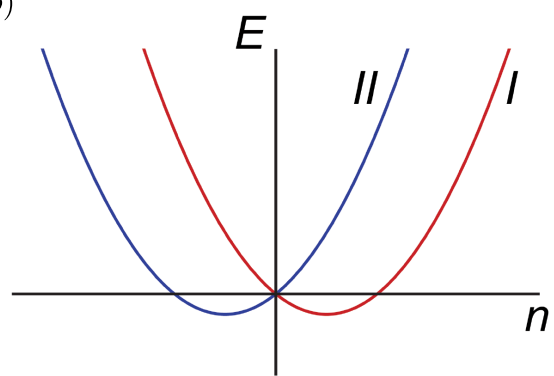

Figure 1.1: (a) Sketch of of the interferometric setup based on a mesoscopic ring with Rashba spin-orbit interaction. (b) Eigenvalues of the ring Hamiltonian of Eq. 1.18 plotted as a function of the quantum number $n$. The two time-reversal channels $I, I I$ are indicated. (Adapted from Ref. [12]).

Eigenstates of the system can be written as,

$$
\Psi^{\uparrow}=e^{i n \phi}\left(\begin{array}{c}
e^{-i \frac{\phi}{2}} \cos \frac{\theta}{2} \\
-e^{i \frac{\phi}{2}} \sin \frac{\theta}{2}
\end{array}\right) \quad \Psi^{\downarrow}=e^{i n \phi}\left(\begin{array}{c}
e^{-i \frac{\phi}{2}} \sin \frac{\theta}{2} \\
e^{i \frac{\phi}{2}} \cos \frac{\theta}{2}
\end{array}\right)
$$

where $\theta=\tan ^{-1} Q_{r}$ is the tilt angle of the electron spin relative to the $\hat{z}$ axis. For $\alpha_{R}=0$ we recover the spin-up and spin-down eigenstates of $\sigma_{z}$. It is important to note that in general the spin eigenstates are not aligned with the effective magnetic field arising from the Rashba spin-orbit coupling (Fig. 1.2a) and are instead characterized by a tilt angle $\theta$. The total geometric phase of the electron travelling across the ring is then given by the solid angle subtended by the total magnetic field (Fig. 1.2b). A complete alignment only happens in the adiabatic limit when $Q_{r} \rightarrow \infty$ and $\theta \rightarrow \frac{\pi}{2}$. To reproduce an interferometric setup (Fig. 1.1a) we can attach two infinite and transparent leads at the two opposite ends of the ring. The electrons injected at $\phi=0$ always come out at $\phi=\pi$. Assuming that the size of the ring is smaller than the mean free path of the electrons in the ring, then the conductance of the system can be calculated by using the Landauer-Buttiker 
(a)

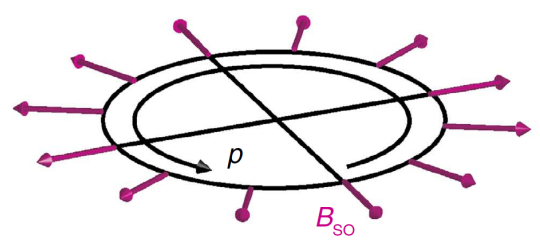

(b)

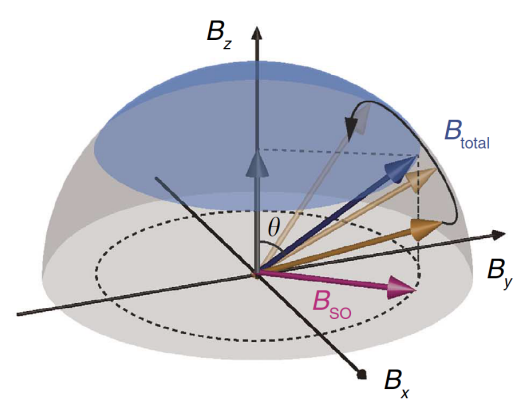

Figure 1.2: (a) The Rashba spin-orbit coupling induces an effective planar magnetic field orthogonal to the momentum of the electron traversing the ring. (b) When transport is non-adiabatic the effective magnetic field has an additional out-of-plane component proportional to $\omega_{0}$. (Adapted from Ref. [8])

formalism [13],

$$
G=\frac{e^{2}}{h} \sum_{\sigma, \sigma^{\prime}} T^{\sigma, \sigma^{\prime}}
$$

where the transmission coefficient $T^{\sigma, \sigma^{\prime}}$ is given by the square of the scalar product between the incoming state and the outgoing state, $T^{\sigma, \sigma^{\prime}}=\left|\left\langle\sigma^{\prime} \mid \sigma_{\text {out }}\right\rangle\right|^{2}$. The outgoing state is written as,

$$
\left|\sigma_{\text {out }}\right\rangle=\frac{1}{4} \sum_{s, \lambda}\left\langle\Psi^{s}(0) \mid \sigma\right\rangle\left|\Psi^{s}(\lambda \pi)\right\rangle
$$

where $\lambda= \pm$ indicates if the state is a right or left mover and the factor $1 / 4$ ensures the correct normalization. The idea behind $\left|\sigma_{\text {out }}\right\rangle$ is that an incoming spin $\sigma$ entering the ring has a certain probability to be transported to the other lead through one of the four available energy channels. These probabilities which are the coefficients of the linear combination of the eigenfunctions calculated in $\pi$ are given by the scalar product of $|\sigma\rangle$ with $\left|\Psi^{s}(0)\right\rangle$. The unpolarized conductance can be written explicitly by summing on a 
complete set of incoming spin states (note that $\sum_{\sigma}|\sigma\rangle\langle\sigma|=\mathcal{I}$ ),

$$
G=\frac{e^{2}}{4 h} \sum_{s, s^{\prime}, \lambda, \lambda^{\prime}}\left\langle\Psi^{s}(0) \mid \Psi^{s^{\prime}}(0)\right\rangle\left\langle\Psi^{s^{\prime}}\left(\lambda^{\prime} \pi\right) \mid \Psi^{s}(\lambda \pi)\right\rangle .
$$

This results in 16 inner products some of which are zero due to the orthogonality between opposite spin states. Finally the overall conductance reads,

$$
\frac{G}{e^{2} / h}=1+\cos \left[\pi Q_{r} \sin \theta-\pi(1-\cos \theta)\right] .
$$

The Rashba phase $\phi_{R}=\pi Q_{r} \sin \theta$ has an additional factor $\sin \theta$ that accounts for the fact that spinors aren't generally aligned with the effective Rashba field. The phase $\phi_{A A}=\pi(1-\cos \theta)$ is the Aharonov-Anandan phase, a non-adiabatic generalization of the Berry phase, related to the solid angle accumulated by the change in spinor orientation during transport (Fig. 1.2). In the adiabatic limit this tends to the $\pi$-Berry phase of the electronic spin $Q_{r} \rightarrow \infty, \phi_{A A} \rightarrow \pi$.

\subsubsection{Berry Phase in Crystals}

The Berry phase naturally appears in crystals where the electronic wavefunctions live in a Brillouin zone which is topologically equivalent to a torus. Assuming negligible interaction effects, the Hamiltonian of a crystal is described by,

$$
\mathcal{H}=\frac{\hat{p}^{2}}{2 m}+V(\mathbf{r})
$$

where $V(\mathbf{r})=V(\mathbf{r}+\mathbf{a})$ is the ionic potential which follows the same periodic structure of the underlying lattice and $\mathbf{a}$ is a Bravais lattice vector. Bloch's theorem ensures that the eigenstates of the crystals can be labelled by a discrete band index $n$ and a crystal wave vector $\mathbf{q}$ which resides inside the Brillouin zone. Moreover the wavefunctions of electrons in the crystal, or Bloch waves, can be written as plane waves modulated by a periodic function,

$$
\psi_{n, \mathbf{q}}(\mathbf{r})=e^{i \mathbf{q} \cdot \mathbf{r}} u_{n, \mathbf{q}}(\mathbf{r})
$$

where $u_{n, \mathbf{q}}(\mathbf{r})$ satisfies the periodic boundary condition $u_{n, \mathbf{q}}(\mathbf{r})=u_{n, \mathbf{q}}(\mathbf{r}+$ a). Considering the unitary transformation $\mathcal{H}(\mathbf{q})=e^{-i \mathbf{q} \cdot \mathbf{r}} \mathcal{H} e^{i \mathbf{q} \cdot \mathbf{r}}$ we see 


\section{Introduction}

that the Brillouin zone is the parameter space of $\mathcal{H}(\mathbf{q})$ and that the states $\left|u_{n, \mathbf{q}}(\mathbf{r})\right\rangle$ are its basis functions. The eigenvalues $\varepsilon_{n, \mathbf{q}}$ define the electronic band structure of the crystal which typically consists of different energy dispersions separated by forbidden energy ranges known as band gaps. If the Fermi energy of the system lies in a band gap then the system is insulating at zero temperature, whereas if the energy bands are only partially filled then the system behaves as a metal.

Now it's clear that if the momentum q can change in momentum space then the Bloch state gathers a Berry phase (also known as Zak phase [14]),

$$
\gamma_{n}=\int_{\mathcal{C}} d \mathbf{q} \cdot\left\langle u(\mathbf{q})\left|i \partial_{\mathbf{q}}\right| u(\mathbf{q})\right\rangle
$$

where $\mathcal{C}$ has to be a loop in the Brillouin zone in order for $\gamma$ to be gaugeinvariant. As usual one can also define a Berry curvature and rewrite the line integral of Eq. 1.29 using Stoke's theorem, $\gamma_{n}=\int d \mathbf{S} \cdot \boldsymbol{\Omega}_{n}(\mathbf{q}) /(2 \pi)^{d}$. If the system is metallic, the energy bands are only partially filled. In this case by applying an external magnetic and/or electric field the electrons on the Fermi surface experience a change in momentum forcing them to loop around the Fermi surface, thus acquiring a non-trivial Berry phase. As we will discuss in the following Section, this Fermi liquid property, known as the anomalous Hall effect [15] is rooted in the anomalous velocity of Bloch electrons. If the system is an insulator, i.e. the Fermi energy lies in an energy gap, the integral of the Berry curvature can be taken over the whole Brillouin zone. As shown in Fig. 1.3, this is equivalent to integrating the curvature over a torus, leading to the same quantization discussed in Section 1.1.1. In this case the effect is a property of the many body insulating state given by the Slater determinant of the occupied bands.

\subsection{Anomalous Velocity}

To show why the Berry curvature is directly responsible for the anomalous velocity of electrons in a crystal, it is first useful to introduce the problem of a one-dimensional crystal subject to a slowly-varying time perturbation. The instantaneous eigenstates of $\mathcal{H}(q, t)$ can still be written in the Bloch form $e^{i q x}\left|u_{n}(q, t)\right\rangle$ since translational symmetry is preserved. At first order 

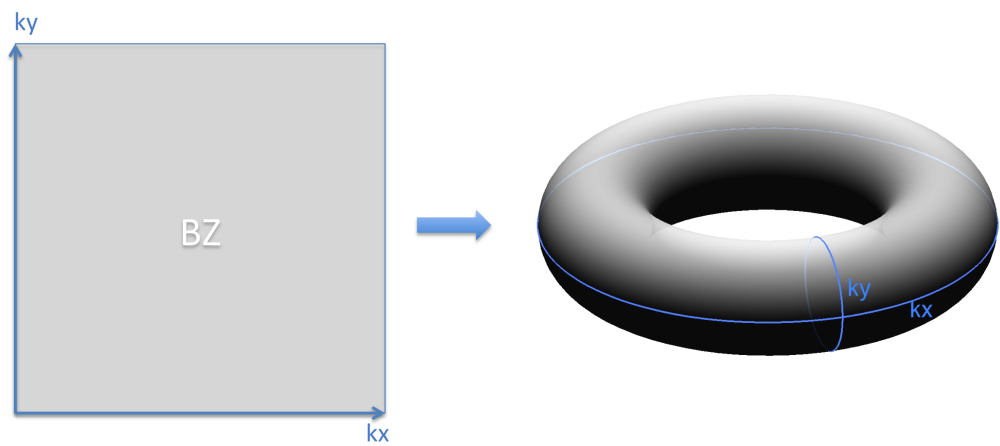

Figure 1.3: The two-dimensional Brillouin zone of a crystal is equivalent to a torus. (Adapted from Ref. [16])

in time-dependent perturbation theory the wavefunction is given by,

$$
\left|u_{n}\right\rangle-i \hbar \sum_{n^{\prime} \neq n} \frac{\left|u_{n^{\prime}}\right\rangle\left\langle u_{n^{\prime}} \mid \partial_{t} u_{n}\right\rangle}{\varepsilon_{n}-\varepsilon_{n}^{\prime}}
$$

The velocity operator can be written in momentum space as $v(q, t)=\partial \mathcal{H}(q, t) / \partial(\hbar q)$. It follows from Eq. 1.30 that at first order the electronic velocity of a state with momentum $q$ given by,

$$
v_{n}(q)=\frac{\partial \varepsilon_{n}(q)}{\hbar \partial q}-i \sum_{n^{\prime} \neq n}\left\{\frac{\left\langle u_{n}\left|\partial_{q} \mathcal{H}\right| u_{n^{\prime}}\right\rangle\left\langle u_{n^{\prime}} \mid \partial_{t} u_{n}\right\rangle}{\varepsilon_{n}-\varepsilon_{n}^{\prime}}-\text { c.c. }\right\} .
$$

Since $\left\langle u_{n}\left|\partial_{q} \mathcal{H}\right| u_{n^{\prime}}\right\rangle=\left(\varepsilon_{n}-\varepsilon_{n}^{\prime}\right)\left\langle\partial_{q} u_{n} \mid u_{n^{\prime}}\right\rangle$ and considering the identity $\sum_{n^{\prime}}\left|u_{n^{\prime}}\right\rangle\left\langle u_{n^{\prime}}\right|=1$ we can recast Eq. 1.31 as,

$$
v_{n}(q)=\frac{\partial \varepsilon_{n}(q)}{\hbar \partial q}-i\left(\left\langle\partial_{q} u_{n} \mid \partial_{t} u_{n}\right\rangle-\left\langle\partial_{t} u_{n} \mid \partial_{q} u_{n}\right\rangle\right) .
$$

The second term is the Berry curvature $\Omega_{n}(q, t)$ where the parameter space is now generated by $(q, t)$. The induced current by the time-dependent perturbation is found upon integration of $v_{n}(q)$ inside the Brillouin zone. Let us consider an insulating system. As the first term disappears, we are left with the integral of $\sum_{n} \int_{B Z} \frac{d q}{2 \pi} \Omega_{n}(q, t)$. If we consider the integration also over a 


\section{Introduction}

period of time $T$ in which the Hamiltonian returns to itself, $\mathcal{H}(t)=\mathcal{H}(t+T)$ the parameter space upon which we are integrating is a torus. As previously mentioned, the integral of the Berry curvature over the Brillouin zone of filled bands, and over the period $T$, i.e $c_{n}=\sum_{n} \int_{0}^{T} \int_{B Z} \frac{d q}{2 \pi} \Omega_{n}(q, t)$, is a quantized value known as the Chern number counting the number of electrons transported from one end of the system to the other during the period of time T. This phenomenon is known as adiabatic charge pumping [17] and is the cornerstone to understanding the integer quantum Hall effect, opening the door to the world of topological insulators.

Let us now consider a three-dimensional crystal subject to a weak external electric field E. We introduce such field in the crystal Hamiltonian by using Peierls substitution,

$$
\mathcal{H}(t)=\frac{(\hat{p}+\mathbf{A}(t))^{2}}{2 m}+V(\mathbf{r}) .
$$

Clearly, this is the same time-dependent problem discussed above. By defining the transformed Hamiltonian $\mathcal{H}(\mathbf{q}, t)=\mathcal{H}(\mathbf{q}+e / \hbar \mathbf{A}(t))$ we can introduce the gauge-invariant crystal momentum $\mathbf{k}=\mathbf{q}+(e / \hbar) \mathbf{A}(t)$ which we can use to label the eigenstates of the system. The time derivative of $\mathbf{k}$ is then given by $\dot{\mathbf{k}}=-(e / \hbar) \mathbf{E}$. By using the identities $\partial_{u_{\alpha}}=\partial_{k_{\alpha}}$ and $\partial_{t}=-(e / \hbar) E_{\alpha} \partial_{k_{\alpha}}$ we can recast Eq. 1.32,

$$
\mathbf{v}_{n}(\mathbf{k})=\frac{\partial \varepsilon_{n}(\mathbf{k})}{\hbar \partial \mathbf{k}}-\frac{e}{\hbar} \mathbf{E} \times \boldsymbol{\Omega}_{n}(\mathbf{k})
$$

where the second term is known as the anomalous velocity. This additional contribution can be thought as the momentum space equivalent of the Lorentz force, where the Berry curvature plays the role of the magnetic field. As such it is always transverse to the electric field and is responsible for different types (quantum/anomalous/spin/valley) of Hall responses in metals and insulators. In Chapter 3 we will show how the anomalous velocity is responsible for a particular type of planar Hall effect in two-dimensional trigonal crystals which has been overlooked so far. 


\subsection{Non-Linear Hall Effect Induced by Berry Curvature Dipole}

Inversion and time-reversal symmetries constrain the Berry curvature to behave respectively as an even and odd function of momentum. When timereversal symmetry is preserved the integral of the Berry curvature is forced to vanish and hence there can be no Hall effects. However, in recent years it was discovered [18] that when considering inversion-broken crystals Halllike currents appear as second order responses to the applied electric field. This can be shown through a semiclassical picture of transport where the current density is given by the integral of the electronic velocity weighed by the distribution function:

$$
j_{a}=e \int_{\mathbf{k}} f(\mathbf{k}) \dot{r}_{a}
$$

with $\int_{\mathbf{k}}=\int d^{d} k /(2 / \pi)^{d}$ and $\partial_{a}=\partial_{k_{a}}$. We will consider for simplicity a single band system and fix $\hbar=1$. The velocity of the Bloch electrons subject to an external electric field is given by Eq. 1.34. Let us now consider an AC electric field $E_{c}(t)=\operatorname{Re}\left\{\mathcal{E}_{c} e^{i \omega t}\right\}$ with $\mathcal{E}_{c} \in \mathbb{C}$. By making use of the relaxation time approximation the Boltzmann equation for the distribution function of the electrons reduces to,

$$
e \tau E_{a} \partial_{a} f+\tau \partial_{t} f=f_{0}-f
$$

where $f_{0}$ is the equilibrium distribution function and $\tau$ is the relaxation time which for simplicity is assumed to be a constant. The response at second order in the electric field can be found by expanding $f$ up to second order, $f=\operatorname{Re}\left\{f_{0}+f_{1}+f_{2}\right\}$ where $f_{n}$ is order $\mathcal{E}^{n}$. Plugging the various $f_{n}$ in Eq. 1.36 a recursive structure appears, yielding:

$$
\begin{aligned}
f_{1} & =f_{1}^{\omega} & f_{1}^{\omega} & =-\frac{e \tau \mathcal{E}_{a} \partial_{a} f_{0}}{1+i \omega t} \\
f_{2} & =f_{2}^{0}+f_{2}^{2 \omega} e^{2 i \omega t} & f_{2}^{0} & =\frac{(e \tau)^{2} \mathcal{E}_{a}^{*} \mathcal{E}_{b} \partial_{a b} f_{0}}{2(1+i \omega t)} \\
f_{2}^{2 \omega} & =\frac{(e \tau)^{2} \mathcal{E}_{a} \mathcal{E}_{\partial_{a b}} f_{0}}{2(1+i \omega t)(1+2 i \omega t)} & &
\end{aligned}
$$




\section{Introduction}

(a) T-R Broken

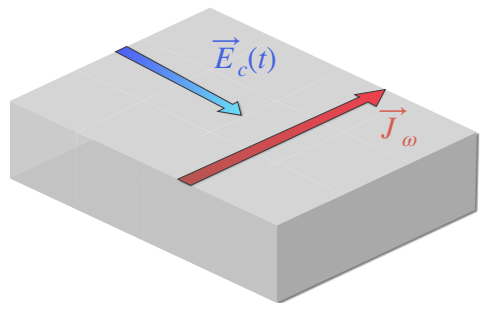

(b) $\quad \underline{\text { T-R Symmetric }}$

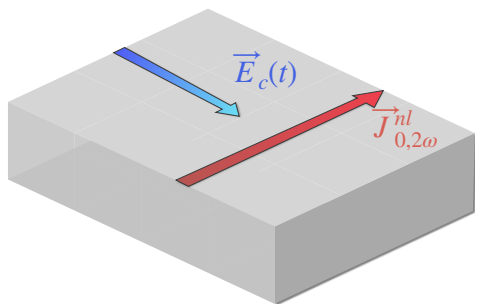

Figure 1.4: Sketch of a conventional Hall response in the absence of time-reversal symmetry (a) and of the non-linear Hall response (b) when timereversal is preserved.

The current density can then be written as the sum of a DC current, and a second harmonic as $j_{a}=R e\left\{j_{a}^{0}+j_{a}^{2 \omega} e^{2 i \omega t}\right\}$, where:

$$
\begin{aligned}
j_{a}^{0} & =\frac{e^{2}}{2} \int_{k} \epsilon_{a b c} \Omega_{b} \mathcal{E}_{c}^{*} f_{1}^{\omega}+e \int_{k} f_{2}^{0} \partial_{a} \varepsilon(k) \\
j_{a}^{2 \omega} & =\frac{e^{2}}{2} \int_{k} \epsilon_{a b c} \Omega_{b} \mathcal{E}_{c} f_{1}^{\omega}+e \int_{k} f_{2}^{2 \omega} \partial_{a} \varepsilon(k) .
\end{aligned}
$$

The second terms of Eq. 1.38 are the semiclassical ones and vanish when assuming time-reversal symmetry and a constant $\tau$. The Berry curvatureinduced quantum part of the non-linear planar Hall effect can instead be written as $j_{a}^{0}=\chi_{a b c} \mathcal{E}_{b} \mathcal{E}_{c}^{*}$ and $j_{a}^{2 \omega}=\chi_{a b c} \mathcal{E}_{b} \mathcal{E}_{c}$ with,

$$
\chi_{a b c}=\epsilon_{a d c} \frac{e^{3} \tau}{2(1+i \omega \tau)} \int_{k}\left(\partial_{b} f_{0}\right) \Omega_{d} .
$$

Hence, the non-linear Hall current appears as the response at zero and twice the frequency of the applied electric field. The presence of the term $\partial_{b} f_{0}$ guarantees that only states near the Fermi surface contribute to the nonlinear current. Hence the response is purely a Fermi liquid property. Partial integration of Eq. 1.39 uncovers the Berry curvature dipole that generates 
this quantum non-linear response,

$$
\chi_{a b c}=-\epsilon_{a d c} \frac{e^{3} \tau}{2(1+i \omega \tau)} \int_{k} f_{0}\left(\partial_{b} \Omega_{d}\right) .
$$

The integral is the first moment of the Berry curvature, known as the Berry curvature dipole:

$$
D_{b d}=\int_{k} f_{0}\left(\partial_{b} \Omega_{d}\right) .
$$

It is interesting to note that for $\omega \tau>>1$ the prefactor of Eq. 1.40 becomes independent of the scattering time. In this limit the non-linear response is entirely determined by the Berry curvature dipole. In two-dimensional crystals the Berry curvature is a pseudoscalar and hence the Berry curvature dipole behaves as pseudovector. It follows that for the dipole to be nonvanishing, the crystal may possess at most one residual mirror symmetry and in this case the dipole will be orthogonal to the mirror line. In Chapter 2 we discuss how strain generates a finite Berry curvature dipole in both monolayer and bilayer graphene due to the warping of the Fermi surface. Moreover in Chapter 3 we show how even in time-reversal broken crystals an anomalous kind of non-linear planar Hall effect can be observed when mirror symmetries are present.

\subsection{Topological Insulators}

As mentioned in Section 1.1.3, insulators are characterized by an energy gap which separates a filled valence band from an empty conduction band. Exciting an electron from the valence to the conductance band costs a finite amount of energy, equal to the band gap at zero temperature, making the system insulating by definition. Of course the size of the energy gap compared to the thermal energy lets us distinguish between insulators and semiconductors, however from a topological classification standpoint both these phases of matter are equivalent to a trivial insulator since they can be deformed into one another by smoothly tuning an external parameter without closing the band gap.

The first non-trivial insulating phase discovered, meaning that it could not be smoothly deformed into a trivial insulator, was the integer quantum Hall state [19]. This exotic phase of matter emerges when applying a strong 


\section{Introduction}

transverse magnetic field to electrons confined in two-dimensions. The magnetic field generates quantized Landau levels with energies $\varepsilon_{n}=\hbar \omega_{c}(n+1 / 2)$, where $\omega_{c}$ is the cyclotron frequency. If the Fermi energy lies in one of the forbidden energy ranges, $N$ Landau levels are filled and the system is by all means an insulator ${ }^{\mathrm{a}}$, When applying an electric field to such a system the cyclotron orbits start to drift and the transverse Hall conductance is quantized to the number of filled Landau levels $\sigma_{x y} /\left(e^{2} / h\right)=N$. As to why the quantum Hall state is not a trivial insulator, the reason is linked to the presence of a non-trivial Chern invariant $n \in \mathbb{Z}$. Indeed, this topological invariant which distinguishes among different classes of insulators. The topological aspect stems from the fact that an insulator with $n=0$ cannot be smoothly deformed (without closing the energy gap) into another one with a different $n$. The Chern number of an occupied band $m$ can be computed by taking the integral of the Berry curvature over the Brillouin zone:

$$
n_{m}=\int_{B Z} d^{2} \mathbf{k} \Omega_{m}(\mathbf{k}) .
$$

The integral of the Berry curvature over a closed manifold is quantized much in the same way as the integral of the gaussian curvature over a closed surface is quantized to the genus of said surfaces, the latter is known as the Gauss-Bonnet theorem. It turns out, however, that it is not necessary to form Landau levels to obtain a quantum Hall state. The Haldane model reproduces the integer quantum Hall effect by considering a honeycomb lattice where time-reversal is broken by a periodic magnetic field that on average is zero. Breaking time-reversal symmetry is sufficient to gap the system; it can then be shown that the Berry curvature contribution of the two low-energy Dirac cones produces a finite Chern number. This effect is known as the quantum anomalous Hall effect. It may be puzzling to see why an insulator exhibits a finite Hall conductance: typically insulators do not conduct. The answer is found by considering what happens at the boundary of a topological insulator, where its interface touches the vacuum or perhaps another trivial insulator (the two are topologically equivalent). It turns out that at the edge of a topological insulator conducting channels appear. These metallic states are the ones responsible for the quantized conductance. The

${ }^{a}$ It is remarkable how the magnetic field completely modifies the physical properties of what would only be a conventional two-dimensional electron gas. 


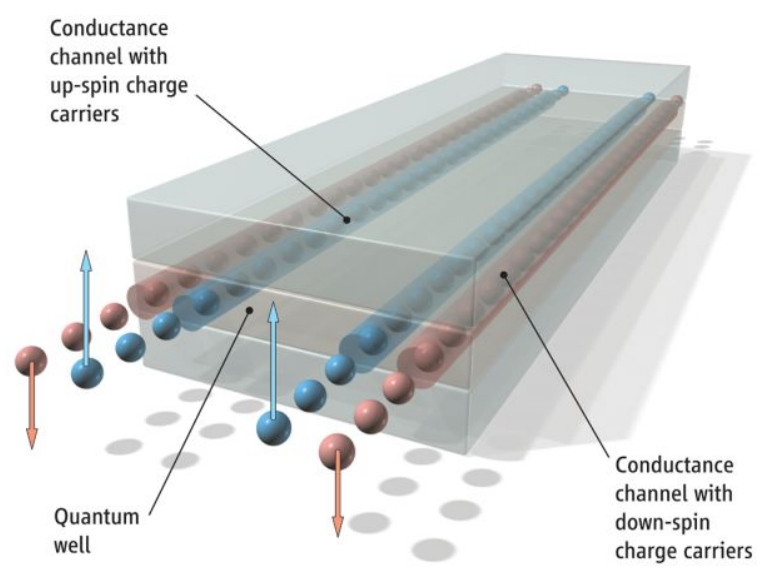

Figure 1.5: Sketch of a quantum spin-Hall insulator and helical edge states in a quantum well. (Adapted from Ref. [22])

edge states are robust against external perturbations since they stem from a topological invariant which is itself unchanged by smooth deformations of $\mathcal{H}$. This phenomenon is known as the bulk-boundary correspondence and its potential technological applications have attracted much interest in various research areas of condensed matter physics.

Since the discovery of the quantum Hall state many other invariants have been uncovered. Depending on the internal and crystalline symmetries of the material different kinds invariants are used for classification. The $\mathbb{Z}_{2}$ topological insulator is among the most notable ones. In contrast with the quantum Hall state, this topological class of insulators preserves time-reversal symmetry. It can be shown that when considering spin-orbit coupled systems non-trivial topology emerges. In two-dimensions this kind of topological insulator is known as the quantum spin-Hall insulator. A minimal model with the necessary features to realize such an insulator was proposed by Kane and Mele [20]. The peculiarity of the quantum spin-Hall insulator is that its edge state are helical, meaning that opposite spins travel in opposite directions along the edges. This phase of matter was later theoretically predicted [21] and experimentally realized [22] in $\mathrm{HgTe} / \mathrm{CdTe}$ quantum wells (Fig. 1.5). In Chapter 5 we make use of the BHZ model to discuss the transport properties of a quantum spin-Hall disk. 


\section{Introduction}

In three dimensions the quantum spin-Hall insulator can be naturally generalized. By using four $\mathbb{Z}_{2}$ topological invariants a three-dimensional topological insulator can be defined [2, 23]. This class of insulators hosts single Dirac cones on their surfaces, a three-dimensional generalization of the discussed bulk-boundary correspondence. However, the presence of an isolated Dirac cone on a surface is an anomaly which at first glance appears to violate the fermion doubling theorem. The solution to this paradox is found by considering the opposite surface which hosts the partner Dirac cone. These metallic surface states are not spin-degenerate and can be effectively thought as half an ordinary metal. In Chapter 6 we discuss how the application of inhomogeneous strain gradients to a three-dimensional topological insulator can couple the metallic surface states and hybridize them, leading to topological phase transitions.

\subsection{Outline}

The thesis is structured as follows:

$\diamond$ Chapter 2 contains a discussion on the appearance of non-linear Hall currents in inversion broken monolayer and bilayer graphene when applying uniform uniaxial strain. We show that the appearance of a finite dipole is rooted in the warping of the Fermi surface.

$\diamond$ In Chapter 3 we show how a previously overlooked anomalous planar Hall effect arises in two-dimensional trigonal crystals as a consequence of the anomalous velocity of the Bloch electrons. We also show that when mirror symmetries constrain this effect to vanish a non-linear counterpart can still be present due to the finite Berry curvature dipole of the system.

$\diamond$ Chapter 4 contains a brief discussion on the anomalous planar Hall effect in a Rashba two-dimensional electron gas. This minimal models typically describes the low energy theory around the high symmetry point $\Gamma$ point of a different number of spin-orbit coupled materials. Typically in time-reversal symmetric conditions the Berry curvature of such model is assumed to be vanishing. However by considering the presence of symmetry allowed warping terms we find that it becomes finite leading to an anomalous planar Hall effect. 
$\diamond$ In Chapter 5 we study the transport properties of a quantum spin-Hall insulator in a disk geometry. We show that the presence of topologically protected helical edge states allow for the control and manipulation of injected polarized spin currents by controlling the Rashba coupling.

$\diamond$ In Chapter 6 we show that the coupling of inhomogeneous strains to the Dirac fermions of three-dimensional topological insulators in thin film geometries results in the occurrence of phase transitions between topologically distinct insulating phases. We find that the topological phase transitions can lead a normal insulator to a topological phase and vice-versa. 



\section{Berry Curvature Dipole in Strained Graphene: a Fermi Surface Warping Effect}

Because of their deep relation to topology $[2,24]$ the family of Hall effects $[1,25]$, the most famous member of which is the quantization of the Hall conductance in strong magnetic fields $[17,19,26]$, have been intensively scrutinized in recent years. A prerequisite for any Hall effect to appear is time-reversal symmetry breaking. Therefore, either magnetic fields or magnetic dopants are required to have a non-vanishing Hall conductance. It has been recently established, however, that a Hall-like current can still be observed in non-centrosymmetric systems as a non-linear response to an external electric field $[18,27]$. Such non-linear Hall current is closely related to the Berry curvature dipole, which is essentially the first moment of the Berry curvature in momentum space. As a result, this non-linear effect can be used as a direct probe of the geometry of the Bloch states in time-reversal invariant systems.

Single-layer transition metal dichalcogenides $\mathrm{MX}_{2}(\mathrm{M}=\mathrm{Mo}, \mathrm{W}$ and $\mathrm{X}=\mathrm{S}$, $\mathrm{Se}, \mathrm{Te})[28]$ have been theoretically predicted $[29,30]$ and experimentally verified [31-33] as material platforms supporting large Berry curvature dipoles. These materials possess large spin-orbit coupling and lack of inversion symmetry in their $T_{d}$ structure. Furthermore, inversion symmetry breaking can be also achieved with the aid of external electric fields in the topological non-trivial $1 T^{\prime}$ phase. A non-linear Hall effect has been also predicted [34] and experimentally observed in bilayer $\mathrm{WTe}_{2}$ [35]. Finally, different noncentrosymmetric three-dimensional materials have been proposed to feature sizable Berry curvature dipoles. These include the topological crystalline insulator SnTe [36], which undergoes a ferroelectric distorsion at low temperatures [37], time-reversal symmetric Weyl semimetals in the TaAs materials class [18], as well as the giant Rashba material BiTeI [38].

The common trait of all these materials is their strong spin-orbit coupling and the presence of low-energy Dirac quasi-particles forming tilted Dirac cones. In particular, the tilt of the Dirac cones does not change the Berry 

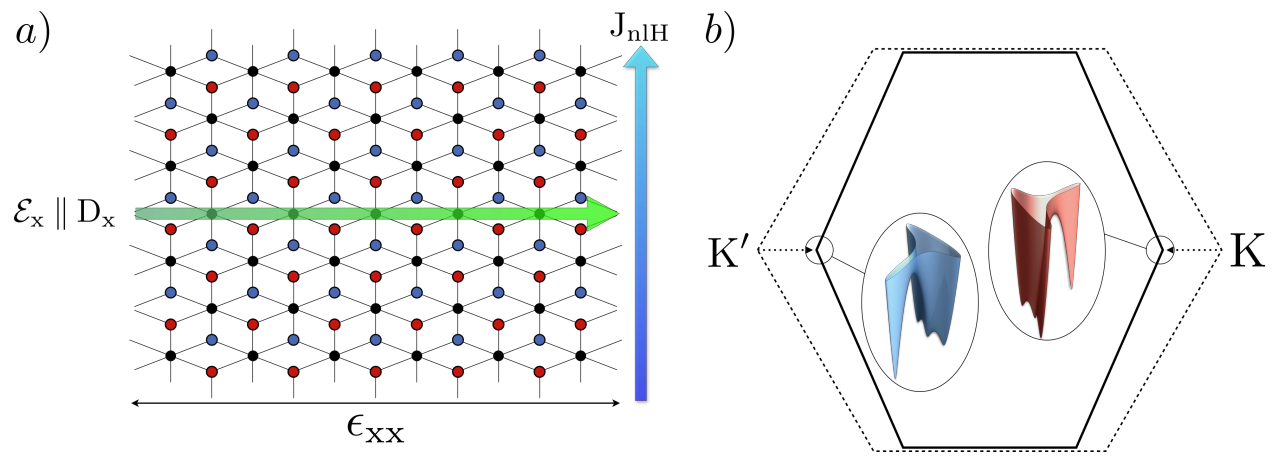

Figure 2.1: a) Top view of the uniformly strained Bernal-stacked bilayer graphene lattice. Red and blue carbon atoms correspond to different layer and are not equivalent due to the applied voltage that breaks inversion symmetry. Black sites indicate the overlap between atoms of the two different layers. By applying an electric field $\mathcal{E}_{x}$ parallel to the dipole $\mathrm{D}_{\mathrm{x}}$, a non-linear Hall current $\mathrm{J}_{\mathrm{nlH}}$ is generated. b) Strain deformation of the Brillouin zone. The warped low-energy dispersion is shown in the vicinity of the two unstrained high symmetry points $K$ and $K^{\prime}$.

curvature of the system but it is crucial to get a corresponding non-vanishing first dipole moment. The aim of this work is to show that this topological effect emerges even in the complete absence of spin-orbit coupling in twodimensional Dirac materials. In these systems, the non-vanishing Berry curvature dipole does not stem from the presence of tilted Dirac cones but it is due to the warping of the Fermi surface. We show indeed that a sizable Berry curvature dipole arises in uniaxially strained single-layer and bilayergraphene where inversion symmetry is broken by the existence of a substrate and an external electric field, respectively. In the absence of shear strains, the non-vanishing dipole in these materials is generated along the zig-zag direction, which is orthogonal to the armchair mirror line (Fig. 2.1a). Even more importantly, the appearance of a finite dipole can only be captured taking explicitly into account the terms accounting for the warping of the Fermi surface (Fig. 2.1b) in the low-energy description of the system. The warping-induced Berry dipole is strongly enhanced in Bernal-stacked bilayer graphene and reaches the nanometer scale, which is comparable to the value experimentally observed in bilayer $\mathrm{WTe}_{2}$ [35]. 


\subsection{Berry Curvature Dipole in Strained Monolayer Graphene}

We start out by recalling the relation between the non-linear Hall current and the Berry curvature dipole as it can be derived using the semiclassical Boltzmann picture of transport [18]. In time-reversal invariant, noncentrosymmetric crystals applying an $\mathrm{AC}$ electric field $E_{c}=\operatorname{Re}\left(\mathcal{E}_{c} e^{i \omega t}\right)$ induces a current $j_{a}=\operatorname{Re}\left(j_{a}^{0}+j_{a}^{2 \omega} e^{2 i \omega t}\right)$. This non-linear current has two Fourier components at zero and twice the frequency of the applied external field, $j^{0}=\chi_{a b c} \mathcal{E}_{b} \mathcal{E}_{c}^{*}$ and $j^{2 \omega}=\chi_{a b c} \mathcal{E}_{b} \mathcal{E}_{c}$. The response function $\chi_{a b c}$, which can be expressed as $\chi_{a b c}=-\epsilon_{a d c} e^{3} \tau D_{b d} / 2(1+i \omega \tau)$, with $\epsilon_{a d c}$ being the Levi-Civita tensor and $\tau$ the scattering time, explicitly contains the Berry curvature dipole $^{\mathrm{a}}$ defined as:

$$
D_{b d}=\int_{k} f_{0}\left(\partial_{b} \Omega_{d}\right)
$$

where $\partial_{a}=\partial_{k_{a}}, \int_{k}=\int d^{d} k /(2 \pi)^{d}$ and $f_{0}$ is the Fermi-Dirac distribution. The Berry curvature $\Omega$ is defined, as usual, as the rotor of the Berry connection $A_{a}=-i\left\langle u_{k}\left|\partial_{a}\right| u_{k}\right\rangle$. Crystalline symmetries may constrain the dipole to be zero: in a two-dimensional crystal, the Berry curvature is a pseudoscalar, and therefore the dipole behaves as a pseudovector contained in the two-dimensional plane. As a result, for a Berry curvature dipole to be non-vanishing the system must have at most one mirror symmetry left. If one mirror is preserved the dipole will then be directed perpendicular to the mirror line.

The wallpaper group of graphene is $p 6 \mathrm{~mm}$, generated by the point group $\mathcal{C}_{6 v}$ and in-plane translations. The point group $\mathcal{C}_{6 v}$ is comprised of a threefold rotation $\mathcal{C}_{3}$, a two-fold rotation $\mathcal{C}_{2}$ and the mirror symmetry $\mathcal{M}_{x}$ [39]. The two-fold rotation $\mathcal{C}_{2}$, which coincides with inversion since spin-orbit coupling can be effectively neglected, is immediately broken assuming a staggered chemical potential between the two honeycomb sublattices (Semenoff mass [40]). The latter can be engineered by placing the graphene sheet on a substrate, for example lattice-matched h-BN [41, 42]. Breaking inversion

${ }^{a}$ Asymmetric defect scattering and other extrinsic mechanisms can also contribute to a non-linear Hall current. These effects can be experimentally decoupled (see Ref.[35]) from the Berry curvature dipole contribution we discuss here. 
reduces the point group to $\mathcal{C}_{3 v}$, gaps out the Dirac cones present at the high symmetry points $K$ and $K^{\prime}$ in the Brillouin zone, and allows for a non-vanishing Berry curvature. To further lower the point group symmetry to $\mathcal{C}_{v}$ we apply a uniform uniaxial strain to the honeycomb lattice along one of the two main crystallographic directions. This uniaxial strain moves the massive Dirac cones away from the the high symmetry points along the $k_{y}=0$ line, as required by the combination of time-reversal $\Theta$ and mirror symmetry $\mathcal{M}_{x}$. The $k_{y}=0$ line is perpendicular to the mirror line and parallel to the dipole. Although the existence of a Berry curvature dipole is perfectly allowed from a symmetry perspective, the corresponding lowenergy theory does not immediately entail the system with a non-vanishing Berry curvature dipole.

To show this we first discuss the low-energy theory of strained graphene. The application of strain to the lattice deforms the primitive cell and the corresponding Brillouin zone (Fig. 2.1b ) but also produces a difference between hopping amplitudes along the two main crystallographic directions. To first order in strain and momentum, the low-energy description of the system shows that the strain behaves effectively as a pseudo-gauge field [43]: $\mathcal{H}_{1}=v_{F}\left[\left(\xi k_{x}+\mathcal{A}_{x}\right) \sigma_{x}+k_{y} \sigma_{y}\right]+\Delta \sigma_{z} / 2$, where $v_{F}$ is the Fermi velocity, $\sigma_{x, y}$ are the Pauli matrices, $\xi$ is the valley degree of freedom, $\Delta$ is the Semenoff mass and $\mathcal{A}_{x} \propto \epsilon_{x x}-\epsilon_{y y}$ is the pseudo-gauge field generated by the strain. If a uniform uniaxial strain is applied to the system, the pure gauge term in $\mathcal{H}_{1}$ can be reabsorbed by performing a momentum shift: this corresponds to expanding the tight-binding Hamiltonian around the Dirac point for $\Delta=0$. However, as was shown in Ref. [44] both through a quantum field theory and a tight-binding approach, the Fermi velocity becomes anisotropic when considering the momentum-strain coupling. The corresponding low-energy Hamiltonian which includes momentum-strain coupling terms is then of the form, $\mathcal{H}_{2}=\xi v_{x} k_{x} \sigma_{x}+v_{y} k_{y} \sigma_{y}+\Delta \sigma_{z} / 2$ where $v_{x}$ and $v_{y}$ are the two straindependent Fermi velocities. The Hamiltonian $\mathcal{H}_{2}$ possesses a finite Berry curvature but still produces a vanishing Berry curvature dipole unless an extra term $\propto \xi k_{x} \sigma_{0}$ is added. This can be seen by performing the integral in Eq. 2.1 as in Ref. [18].

We will now show that the warping of the Fermi surface generates a finite Berry curvature dipole regardless of the presence of a tilt mechanism. By taking into account the trigonal warping caused by the $k^{2}$ terms the effective Hamiltonian to second order in momentum and first order in strain is, 


$$
\begin{aligned}
\mathcal{H}_{\text {warped }}= & \xi v_{x} k_{x} \sigma_{x}+v_{y} k_{y} \sigma_{y}+\frac{\Delta}{2} \sigma_{z}+ \\
& +\left(\lambda_{1} k_{y}^{2}-\lambda_{2} k_{x}^{2}\right) \sigma_{x}+2 \xi \lambda_{3} k_{x} k_{y} \sigma_{y}
\end{aligned}
$$

where $\lambda_{1,2,3}$ are the warping terms which in general are not equivalent when considering strain-momentum coupling terms $\mathcal{O}\left(\epsilon k^{2}\right)$. The Hamiltonian in Eq. 2.2 can be derived from a low-energy expansion of the tightbinding model following similar steps to the ones in Ref. [44] and the Supplemental Material. It is important to note that although Eq. 2.2 has anisotropic warping terms, for a non zero-dipole to exist it is sufficient to consider anisotropic velocities and a $\mathcal{C}_{3}$ symmetric warping. We will make use of this result later on when discussing bilayer graphene.

As shown in Fig. 6.1, when applying strain the warped surface deforms shifting the dipole moment from zero to a finite value leading to a Berry curvature dipole running along the zig-zag direction. Therefore when applying an electric field $E$ along the dipole direction a non-linear Hall current $\mathcal{O}\left(E^{2}\right)$ is generated along the armchair direction. We have computed the Berry curvature dipole generated by the two cones, which contribute equally to the overall response, by continuously changing the carrier density $n$ and different values of strain. At $5 \%$ strain and for a Semenoff mass $\Delta=20 \mathrm{meV}$ a dipole $\mathrm{D}_{\mathrm{x}} \approx 10^{-3} \mathrm{~nm}$ is found at an electron density $n \approx 10^{10} \mathrm{~cm}^{-2}$. This order of magnitude of $D_{x}$ is comparable to the one predicted in Ref. [18] for TMDs.

\subsection{Berry Curvature Dipole in Strained Bilayer Graphene}

Having established that uniaxially strained monolayer graphene possesses a sizable Berry curvature dipole, we now show that the same effect persists also in bilayer graphene in the $(\mathrm{AB})$ Bernal-stacked structure. Importantly, the Berry dipole in this material is boosted by over three order of magnitudes. Moreover, in bilayer graphene inversion symmetry breaking can be achieved with the application of an external electric field perpendicular to the layers. The electric field, in fact, generates a spectral gap $\Delta$ and lowers the point group symmetry from $D_{3 d}$ to $C_{3 v}$ [45]. Notice that this inversion symmetry breaking mass can be experimentally tuned independent of the 
a)

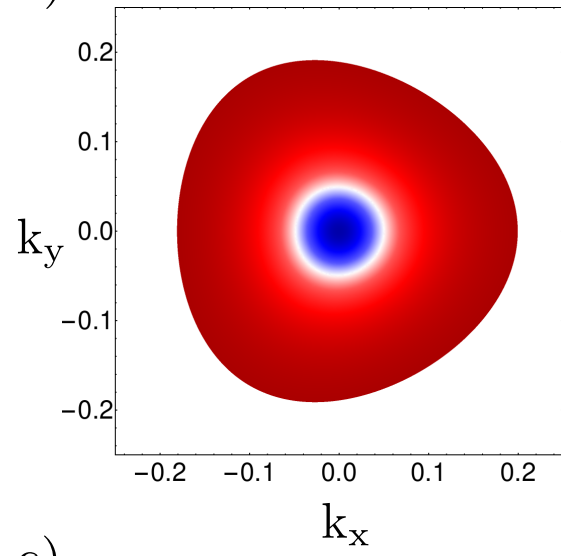

c)

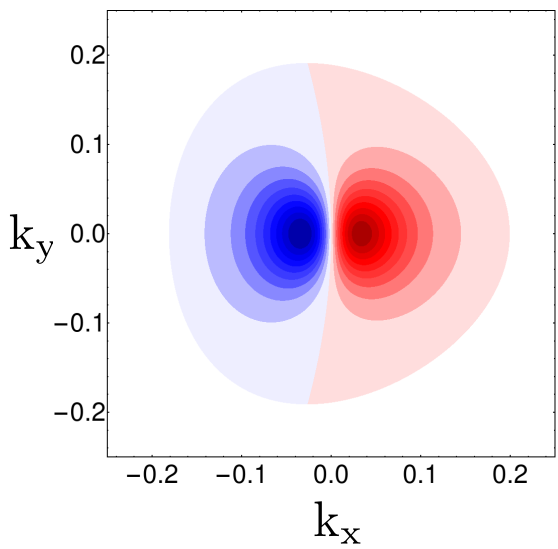

b)

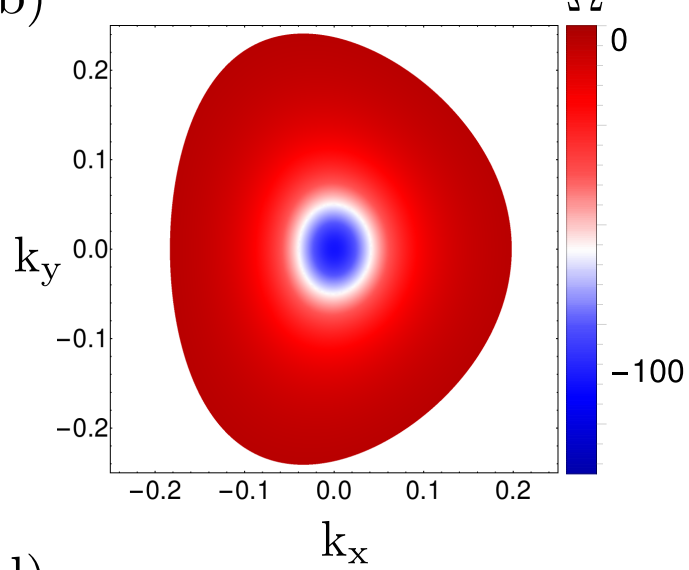

d)

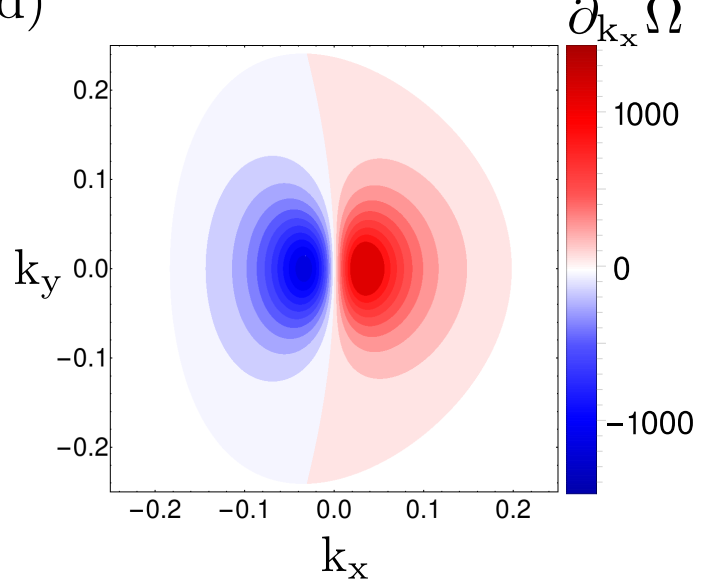

Figure 2.2: Berry curvature $\Omega$ and dipole density $\partial_{k_{x}} \Omega$ of the conduction band corresponding to the Hamiltonian of Eq. 2.2 for unstrained (a,c) and strained $(b, d)$ monolayer graphene. When strain is present the Fermi surface is deformed shifting the dipole moment from zero to a finite value. Momenta are measured in units of the inverse of the lattice constant $a$; the Berry curvature in units of $a^{2}$ while the dipole density in units of $a^{3}$. 
carrier density [46]. The additional application of a uniaxial strain reduces the point group to $C_{v}$ and yields a vanishing Berry dipole perpendicular to the mirror line. To prove the assertion above, we introduce a low-energy effective Hamiltonian [47], valid for electron densities up to $n \approx 10^{13} \mathrm{~cm}^{-2}$ [48, 49], explicitly accounting for the effect of strain [50], reading:

$$
\begin{array}{r}
\mathcal{H}_{b}=\left[-\frac{1}{2 m}\left(k_{x}^{2}-k_{y}^{2}\right)+\xi v_{3} k_{x}+w\right] \sigma_{x} \\
-\left(\frac{1}{m} k_{x} k_{y}+\xi v_{3}\right) \sigma_{y}+\frac{\Delta}{2} \sigma_{z} .
\end{array}
$$

In the equation above, $\xi= \pm 1$ is the valley index, $v_{3}$ is the Fermi velocity related to the "skew" hopping between the layers, whereas $m$ is an effective mass directly dependent on the interlayer coupling. Finally, $w=\mathcal{A}_{3}-\mathcal{A}_{0}$ is the strain term in the Hamiltonian which can be expressed in terms of the two pseudo-gauge fields $\mathcal{A}_{0,3}$. In the presence of inversion symmetry $(\Delta=0)$, the strain-free $(w=0)$ system features a Lifshitz transition [48] at energy $\varepsilon_{L}=m v_{3}^{2} / 2(\approx 1 \mathrm{meV})$ where the Fermi surface splits from a single connected pocket into four different ones: the electronic dispersion consists of one central Dirac cone with $-\pi(\pi)$ Berry phase at the $K\left(K^{\prime}\right)$ point of the Brillouin zone and three "leg" Dirac cones, each of which carries a $\pi(-\pi)$ Berry phase. Notice that the distance between the different cones defines a characteristic momentum $\kappa_{L}=m v_{3} / \hbar\left(\approx 0.035 \mathrm{~nm}^{-1}\right)$. The effect of the strain on the inversion symmetric system is twofold, as it moves the Dirac cones away their unstrained positions and it promotes changes in the topology of the Fermi surface by merging the cones together. For $-1 \leq w / \varepsilon_{L} \leq 3$ there are always four Dirac points, two on the $k_{y}=0$ line and the remaining two in a symmetric position with respect to it. At $w=-\varepsilon_{L}$ the Dirac cones on the invariant line merge and become gapped, giving rise to a local minimum in the dispersion relation which survives until $w \geq-9 \varepsilon_{L}$, after which it becomes a saddle point. For $w>3 \varepsilon_{L}$, instead, there are only the cones on the $k_{y}=0$ line and no other local minima. However, unless $\Delta \neq 0$, the spectrum remains gapless and the Berry curvature is zero. As mentioned above, these gapless spectra become gapped with an externally applied electric field. Consequently, the Dirac points become local minima of the dispersion. 


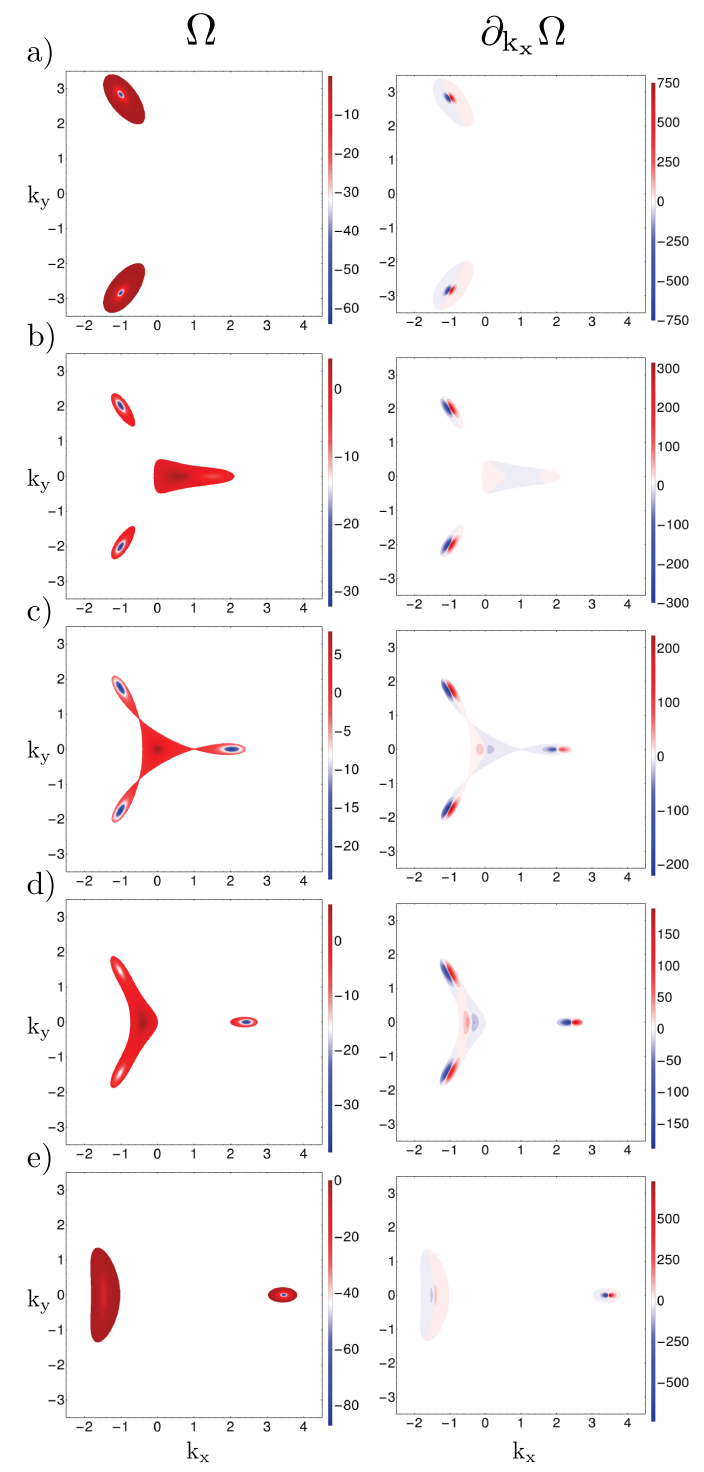

Figure 2.3: Berry curvature $\Omega$ and dipole density $\partial_{k_{x}} \Omega$ of the conduction band corresponding to the low-energy Hamiltonian of Eq. 2.3 for different values of the strain: a) $w=-5 \varepsilon_{L}$, b) $w=-1 \varepsilon_{L}$, c) $w=0$, d) $w=1 \varepsilon_{L}$, e) $w=5 \varepsilon_{L}$. All plots are shown for the same carrier density fixed by placing the Fermi energy $E_{F}$ at the Lifschitz transition in the unstrained $w=0$ and gapped case (panel c). Momenta are measured in units of $\kappa_{L}$, the Berry curvature in units of $1 / \kappa_{L}^{2}$, and the dipole density in units of $1 / \kappa_{L}^{3}$. 


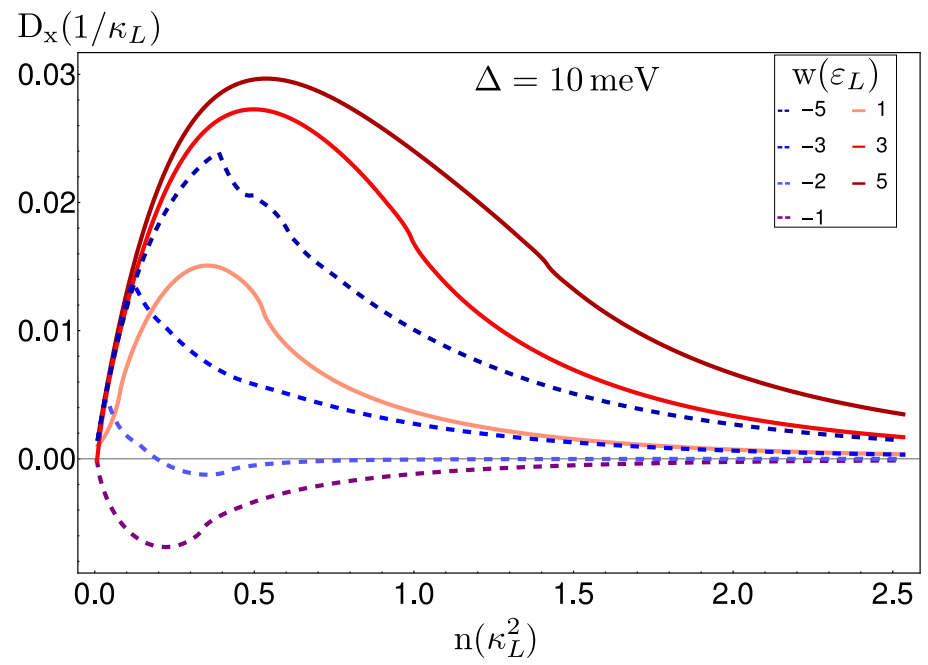

Figure 2.4: Berry curvature dipole, measured in units of $1 / \kappa_{L}$, in bilayer graphene for $\Delta=10 \mathrm{meV}$ and various strains $w$ as a function of the electron density $n$ measured in units of $\kappa_{L}^{2}$ where $\kappa_{L} \simeq 0.035 \mathrm{~nm}^{-1}$. Densities of this order of magnitude have been experimentally reported in Ref.s $[46,51,52]$.

The possible topologies of the Fermi surface induced by the strain are visible in Fig. 2.3, where we plot the Berry curvature and the density of Berry curvature dipole at different values of $w$ and $\Delta \neq 0$. We notice that the plots are symmetric for the exchange $k_{y} \rightarrow-k_{y}$, as dictated by the combination of time-reversal and mirror symmetry. The unstrained case is shown in Fig. 2.3(c). The threefold $\mathcal{C}_{3}$ rotation symmetry constrains the total Berry dipole to be zero in this case.

It is crucial to notice, however, that while the central gapped Dirac cone has a vanishing Berry dipole, the three leg gapped Dirac cones have a nonzero dipole when taken by themselves. This is because each of the leg gapped Dirac cones can be described with an effective low-energy Hamiltonian of the form of Eq. 2.2 with $\lambda_{1}=\lambda_{2}=\lambda_{3}$. The perfect cancellation of these three non-zero contributions due to the threefold rotation symmetry is lost in the presence of uniaxial strain. Moreover, in the presence of finite strain also the central gapped cone yields a non-zero contribution to the Berry dipole. Figs. 2.3(a-b,d-e) actually suggest that when strain deforms the cones, a 
net Berry curvature dipole is generated. In order to verify this, we have computed the Berry dipole as a function of the electron density for different values of $w$ [see Fig.2.4]. The mass has been chosen as $\Delta=10 \varepsilon_{L}$.

The behavior of the total Berry dipole has a richer structure as compared to the one for monolayer graphene: it indeed shows cusps and inflection points that are a different consequence of the different Lifshitz transitions and reflect the richer Fermiology of bilayer graphene. Furthermore, in this material it is possible to tune the strain in such a way that the sign of the dipole changes upon increasing the electron density with an external gating [46], thus inverting the direction of the transverse current or even suppressing it altogether. More importantly, at $w=-5 \varepsilon_{L}$, which corresponds roughly to a $1 \%$ strain [50], and for a gap $\Delta=10 \mathrm{meV}$, we find for $n \approx 10^{11} \mathrm{~cm}^{-2}$ [46] a maximum dipole strength of $\mathrm{D}_{\mathrm{x}} \approx 1 \mathrm{~nm}$. This value is three-order of magnitudes larger than that of single-layer graphene, and is comparable to the Berry dipole experimentally found in bilayer $\mathrm{WTe}_{2}$ [35]. As shown in the Supplemental Material, even higher values on the tens of nanometer scale can be found by decreasing the inversion symmetry breaking mass.

\subsection{Conclusions}

To wrap up, we have shown that non-vanishing Berry curvature dipoles can emerge even in the complete absence of spin-orbit coupling in twodimensional Dirac materials as a result of the warping of the Fermi surface. We have in fact proved that, in the presence of substrate-induced and gate-induced band gaps respectively, uniaxially strained monolayer and Bernal-stacked bilayer graphene do possess sizeable Berry curvature dipoles. In the bilayer structure, the Berry dipole is strongly enhanced and its value is comparable to the one experimentally observed in bilayer $\mathrm{WTe}_{2}$. Since the warping of the Fermi surface is ubiquitous, we expect that our results apply to a large number of two-dimensional materials where strain engineering can be used to achieve the minimum symmetry constraints for a non-vanishing Berry curvature dipole. The corresponding non-linear Hall effect can thus be used as a way to directly probe the geometric properties of Bloch states in a large number of time-reversal invariant two-dimensional materials. 


\section{A Appendix}

\section{A.1 Monolayer Graphene}

\section{Low Energy Hamiltonian}

To derive the Hamiltonian $\mathcal{H}_{\text {warped }}$ of Eq. 2 of the main text we start by considering a spinless nearest-neighbour tight-binding Hamiltonian for graphene,

$$
\mathcal{H}_{t b}=\sum_{\mathbf{k}} \psi_{\mathbf{k}}^{\dagger} \mathcal{H}_{t b}(\mathbf{k}) \psi_{\mathbf{k}}
$$

where we defined the spinor $\psi_{\mathbf{k}}=\left(a_{\mathbf{k}}, b_{\mathbf{k}}\right)$ with $a_{\mathbf{k}}$ and $b_{\mathbf{k}}$ the two fermionic annihilation operators that destroy an electron with momentum $k$ on the sublattice $A$ and $B$ respectively. We also introduced the Bloch Hamiltonian,

$$
\mathcal{H}_{t b}(\mathbf{k})=\left(\begin{array}{cc}
\frac{\Delta}{2} & f(\mathbf{k}) \\
f^{*}(\mathbf{k}) & -\frac{\Delta}{2}
\end{array}\right)
$$

where $f(\mathbf{k})=-\sum_{n} t_{n} e^{i \mathbf{k} \cdot \delta_{n}}$ and $\Delta$ is the Semenoff mass. The positions $\delta_{n}$ of the three nearest $\mathrm{B}$ atoms relative to a given atom $\mathrm{A}$ are written as $\delta_{1}=(0, a / \sqrt{3}), \delta_{2}=(a / 2,-a / 2 \sqrt{3}), \delta_{3}=(a / 2, a / 2 \sqrt{3})$ where $a$ is the lattice constant. When applying strain to the system nearest-neighbour hoppings are renormalized[44]: to first order in strain the hopping has the form $t_{n}=t_{0}\left(1-\beta \Delta u_{n}\right)$, where $\Delta u_{n}$ is the change in distance between two adjacent carbon atoms, $\beta=|\partial \log t / \partial \log a|$ is a material dependent parameter (in graphene[43] $\beta \approx 2$ ). The variation $\Delta u_{n}$ is expressed in terms of the strain tensor $\epsilon_{i, j}$ as $\Delta u_{n}=3 \delta_{n}^{i} \epsilon_{i, j} \delta_{n}^{j} / a^{2}$ with $\delta_{n}^{i}$ being the i-th component of the $\mathrm{n}$-th nearest-neighbour vector. A low energy description of strained graphene can be obtained by expanding $f(\mathbf{k})$ around one of the two inequivalent valleys of the Brillouin zone $\mathbf{K}_{ \pm}=( \pm 4 \pi / 3 a, 0)$. To keep the warping effects we expand up to second order in momentum and keep the momentum-strain coupling terms up to $\mathcal{O}\left(\epsilon k^{2}\right)$. The resulting low energy Hamiltonian for $\mathbf{q}=\mathbf{k}+\mathbf{K}_{ \pm}$is, 


$$
\begin{aligned}
\mathcal{H}^{\prime}(\mathbf{q})= & v_{F} A_{x} \sigma_{x}+\xi v_{x} q_{x} \sigma_{x}+v_{y} q_{y} \sigma_{y}+ \\
& +\left(\lambda_{1} q_{y}^{2}-\lambda_{2} q_{x}^{2}\right) \sigma_{x}+2 \xi q_{x} q_{y} \lambda_{3} \sigma_{y}+\frac{\Delta}{2} \sigma_{z}
\end{aligned}
$$

where we defined $v_{F}=\sqrt{3} t_{0} a / 2, A_{x}=\sqrt{3} \beta\left(\epsilon_{x x}-\epsilon_{y y}\right) / 2 a, v_{x}=v_{F}[1-$ $\left.\beta\left(3 \epsilon_{x x}+\epsilon_{y y}\right) / 4\right], v_{y}=v_{F}\left[1-\beta\left(\epsilon_{x x}+3 \epsilon_{y y}\right) / 4\right], \lambda_{0}=t_{0} a^{2} / 8, \lambda_{1}=\lambda_{0}[1-$ $\left.\left.\beta\left(3 \epsilon_{x x}+\epsilon_{y y}\right) / 4\right]\right), \lambda_{2}=\lambda_{0}\left[1-\beta\left(5 \epsilon_{y y}-\epsilon_{x x}\right) / 4\right], \lambda_{3}=\lambda_{0}\left[1-\beta\left(3 \epsilon_{x x}+\epsilon_{y y}\right) / 4\right]$. To obtain $\mathcal{H}_{\text {warped }}$ of Eq. 2 of the main text, it is sufficient to then perform a momentum shift $q_{x} \rightarrow q_{x}^{\prime}+A_{x}$ in $\mathcal{H}^{\prime}$. Moreover, in order to account for all terms of the order $\mathcal{O}\left(\epsilon k^{2}\right)$ also cubic terms should be added to expansion since terms $q_{x, y}^{2} q_{y, x}$ and $q_{x}^{3}$ will contribute when performing the shift.

\section{Berry Curvature Dipole}

The Berry curvature dipole has been computed at zero temperature such that $f_{0}(\epsilon)=\theta\left(\epsilon-\epsilon_{F}\right)$. In Fig. 2.5 we show $\mathrm{D}_{\mathrm{x}}$ for various values of the Semenoff mass $\Delta$ and the strain $\epsilon_{x x}$ as a function of the electron density $n$ taking into account both the contributions from the two valleys and the two spin species. The dipole changes sign when inverting $\epsilon_{x x}$ (which corresponds to dilating or compressing the lattice along the main crystallographic directions) but is not odd in strain.

\section{A.2 Bilayer Graphene}

\section{Effective $2 \times 2$ Hamiltonian}

Strain effects in bilayer graphene can be monitored using the low-energy effective $\mathbf{k} \cdot \mathbf{p}$ Hamiltonian,

$$
\mathcal{H}_{4 \times 4}=\left(\begin{array}{cccc}
-\frac{\Delta}{2} & v_{0} \pi^{\dagger}+\mathcal{A}_{0} & 0 & v_{3} \pi+\mathcal{A}_{3} \\
v_{0} \pi+\mathcal{A}_{0} & -\frac{\Delta}{2} & \gamma_{1} & 0 \\
0 & \gamma_{1} & \frac{\Delta}{2} & v_{0} \pi^{\dagger}+\mathcal{A}_{0} \\
v_{3} \pi^{\dagger}+\mathcal{A}_{3} & 0 & v_{0} \pi+\mathcal{A}_{0} & \frac{\Delta}{2}
\end{array}\right)
$$

where $\pi=k_{x}+i k_{y}, v_{0 / 3}$ are two strain independent parameters with the dimension of a velocity and the strain appears as two gauge fields $\mathcal{A}_{0 / 3}$. 


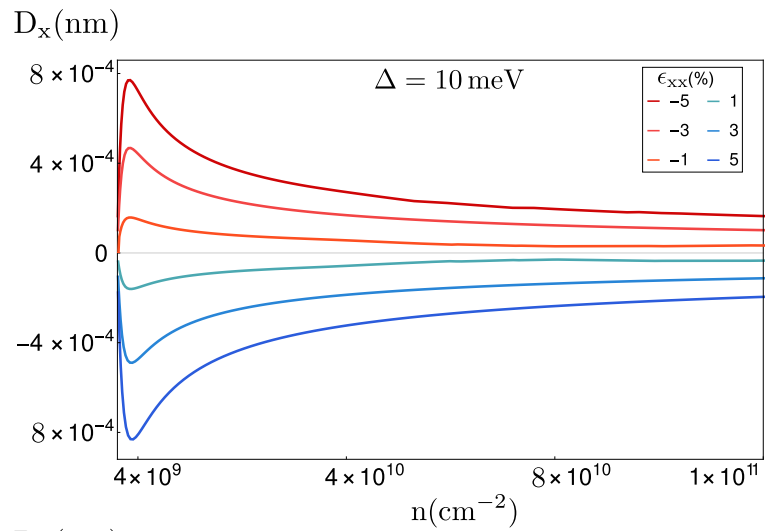

$\mathrm{D}_{\mathrm{x}}(\mathrm{nm})$

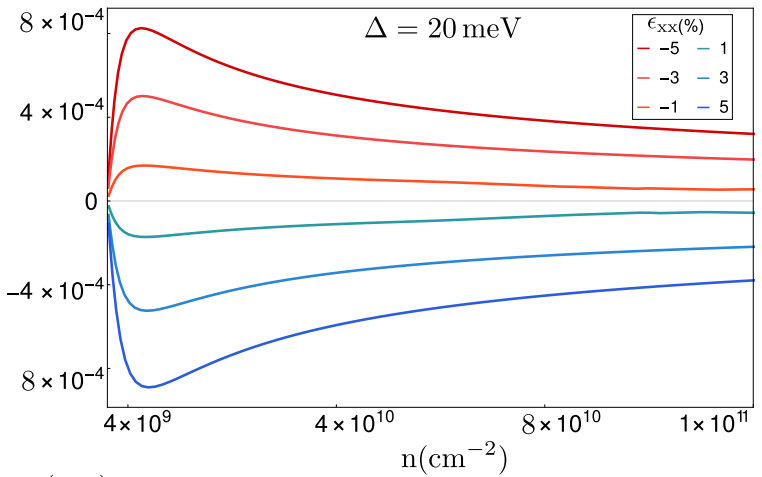

$\mathrm{D}_{\mathrm{x}}(\mathrm{nm})$

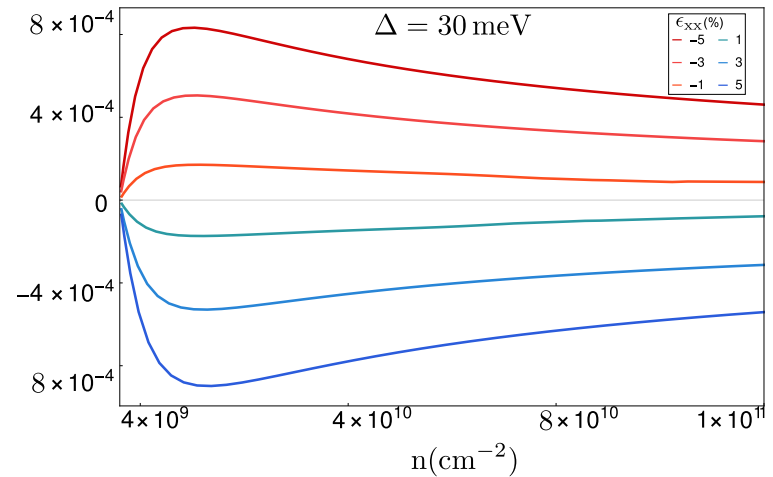

Figure 2.5: Berry curvature dipole of strained monolayer graphene for different values of the Semenoff mass $\Delta$ and the strain $\epsilon_{x x}$ as a function of the electron density $n$. 
The microscopic derivation of the above Hamiltonian starts from a $4 \times$ 4 tight binding model that includes the $2 \mathrm{p}_{z}$ orbitals of the four atoms in the unit cell [45] coupled by the intralayer, interlayer and 'skew' hopping amplitudes $\gamma_{0}, \gamma_{1}$ and $\gamma_{3}$. As for single layer graphene, strain is incorporated in the model up to the first order by modifying the hopping parameters and deforming the primitive cell. The resulting Hamiltonian is then Taylor expanded up to first order around the $K$ point of the strained Brillouin zone to give Eq. 2.6, with $v_{0 / 3}=\sqrt{3} a \gamma_{0 / 3} / 2 \hbar$ and $\mathcal{A}_{0 / 3}=\frac{3}{4} \gamma_{0 / 3}\left(\epsilon_{x x}-\epsilon_{y y}\right) \beta_{0 / 3}$. In bilayer graphene the lattice constant is $a=\sqrt{3} a_{C C}=0.246 \mathrm{~nm}$, where $a_{C C}=$ $0.142 \mathrm{~nm}$ is the carbon-carbon distance. For energies near the Fermi level the orbitals coupled by $\gamma_{1}$ can be eliminated $[48,49]$ through a Schrieffer-Wolff transformation [47] and the small momenta and small strain four band model is replaced, after a gauge transformation $k_{x} \rightarrow k_{x}-\mathcal{A}_{0}$, by the effective $2 \times 2$ Hamiltonian [50],

$$
\begin{array}{r}
\mathcal{H}_{b}=\left[-\frac{1}{2 m}\left(k_{x}^{2}-k_{y}^{2}\right)+\xi v_{3} k_{x}+w\right] \sigma_{x} \\
-\left(\frac{1}{m} k_{x} k_{y}+\xi v_{3}\right) \sigma_{y}+\frac{\Delta}{2} \sigma_{z} .
\end{array}
$$

Here $m=\gamma_{1} / 2 v_{0}^{2}$ and $w=\frac{3}{4} \gamma_{3}\left(\epsilon_{x x}-\epsilon_{y y}\right)\left(\beta_{3}-\beta_{0}\right)$. For $w=0$ the dispersion relation exhibits a Lifshitz transition of the Fermi surface at the energy[45] $\varepsilon_{L}=m v_{3}^{2} / 2(\approx 1 \mathrm{meV})$. It is therefore natural to express the energies in units of $\varepsilon_{L}$ and the momenta in the related scale $\kappa_{L}=m v_{3} / \hbar(\approx 0.035$ $\left.\mathrm{nm}^{-1}\right)$.

\section{Berry Curvature Dipole}

Here we show the Berry dipole in the BLG for different values of the gap. As expected the maximum attainable dipole depends in nonmonotonous manner from the gap. The Berry curvature dipole has been computed at zero temperature such that $f_{0}(\epsilon)=\theta\left(\epsilon-\epsilon_{F}\right)$. In Fig. 2.6 we show $\mathrm{D}_{\mathrm{x}}$ for various values of the Semenoff mass $\Delta$ and the strain $\epsilon_{x x}$ as a function of the electron density $n$ taking into account both the contributions from the two valleys and the two spin species. 

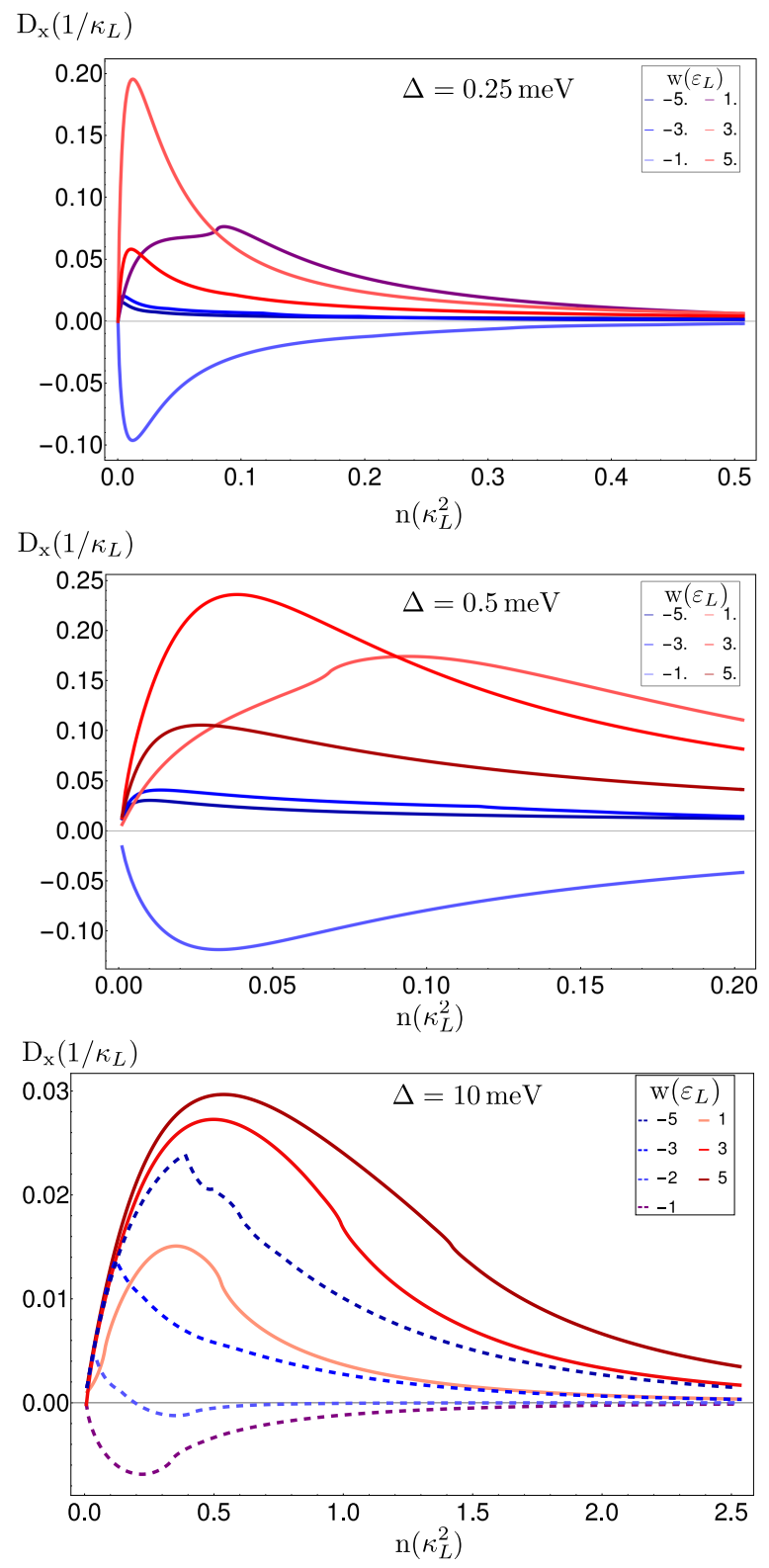

Figure 2.6: Berry curvature dipole of strained bilayer graphene for different values of the gap $\Delta$ and the strain $w$ as a function of the electron density $n$. 



\section{Anomalous Planar Hall Effect in Two-Dimensional Trigonal Crystals}

The Hall effect arises when the conduction electrons of a solid acquire a transverse velocity either due to an externally applied magnetic field or an intrinsic ordered magnetic structure. The associated Hall conductivity is encoded in the antisymmetric dissipationless part of the conductivity tensor, which, for a two-dimensional system, is given by the single scalar $\sigma_{\mathrm{H}}=\left(\sigma_{x y}-\sigma_{y x}\right) / 2$. Onsager reciprocity relations force $\sigma_{\mathrm{H}}$ to vanish in time-reversal symmetric conditions. In addition $\sigma_{\mathrm{H}}$ transforms as a pseudoscalar under a generic spatial point-group symmetry operation. Hence to observe a Hall response it is necessary to break, beside the time-reversal invariance, all mirror symmetries. These conditions are immediately met in the ordinary classical Hall effect where an out-of-plane magnetic field is applied. In this configuration a net Lorentz force grants the electron a transverse velocity and consequently a finite Hall voltage. On the other hand, a magnetic field coplanar with the driving electric field cannot generate a Lorentz force bending the electron trajectories. Nevertheless, transverse currents can and do still exist in strongly spin-orbit coupled systems displaying a sizable anisotropy in the magnetoconductance. This magnetotransport phenomenon, known as planar Hall effect (PHE), does not contribute to the dissipationless Hall conductivity $\sigma_{\mathrm{H}}$, but manifests itself in the symmetric contribution of the conductivity tensor: it cannot be qualified as a genuine Hall effect. In the majority of (quasi)-two-dimensional systems, the PHE has an entirely semiclassical origin and has been shown to arise in thin films of ferromagnetic semiconductors [53-55] and two-dimensional electron gases formed at perovskite oxide interfaces $[56,57]$. Band anisotropies have also been proposed as the source of the PHE in thin films of antiferromagnetic semiconductors [58]. Moreover the PHE plays a central role in the transport properties [59-61] of Weyl semimetals [37, 62-70]. In these topological semimetals, the induced transverse Hall voltage, the applied current, and the magnetic field all lie in the same plane, precisely in a configuration in which the conventional Hall effect 


\section{Anomalous Planar Hall Effect in Two-Dimensional Trigonal Crystals}

vanishes. Even more importantly, the PHE in Weyl semimetals is a prime physical consequence of the chiral anomaly of Weyl fermions [71-76]. The conducting surfaces of three-dimensional topological insulators (3DTI) [2] have also been recently shown to support a PHE [77, 78]. In these materials, an external planar magnetic field conspires with the spin-momentum locking of the Dirac cones to produce a strongly directional dependent net transverse current. All these studies established a paradigm for the planar Hall effect: i) a $\sin 2 \theta$ angular dependence with $\theta$ representing the relative angle between the applied electric and magnetic fields and ii) a magnitude set precisely by the anisotropy in the longitudinal magnetoresistance. However, a PHE beyond this paradigm is in principle symmetry allowed. Beside time-reversal invariance, a planar magnetic field can potentially break all mirror symmetries present in the solid state structure. Therefore, a planar magnetic field is entitled to generate a dissipationless Hall conductance.

In this study, we demonstrate that two-dimensional (2D) materials with strong spin-orbit coupling and crystalline trigonal symmetry possess a previously overlooked anomalous planar Hall effect (APHE). This effect unique to trigonal crystals, derives directly from the "bending" of the electron trajectories encoded in the geometric properties of the electronic wavefunctions [1] - the APHE stems from a Zeeman-induced non-trivial Berry curvature profile. Besides possessing the antisymmetric properties of conventional Hall conductivities, i.e. $\sigma_{x y} \rho_{y x}=-1$, we show that the APHE is independent of the relative direction between the driving electric field and the in-plane magnetic field. Therefore, as shown in Fig. 3.1, these anomalous planar Hall currents persist even when the two fields are collinear and the conventional planar Hall currents vanish. We also show that when mirror symmetries constrain the APHE to vanish (Fig. 3.1), transverse Hall currents are still present: they arise in the non-linear response regime and manifest as a second harmonic response to an oscillating electric field. In strict analogy with the non-linear Hall effect of time-reversal invariant materials [18, 27, 29$32,34,35,38,79,80]$, we find that this non-linear APHE has a geometric contribution that is directly related to the first moment of the Berry curvature, the so-called Berry curvature dipole [18]. This clearly distinguishes the non-linear response we discuss here with the one recently shown to exist on the surface of 3DTIs [78].

We propose graphene and transition metal dichalcogenides (TMD) monolayers [29, 81, 82], where transition metal and chalcogen atoms form trigonal 


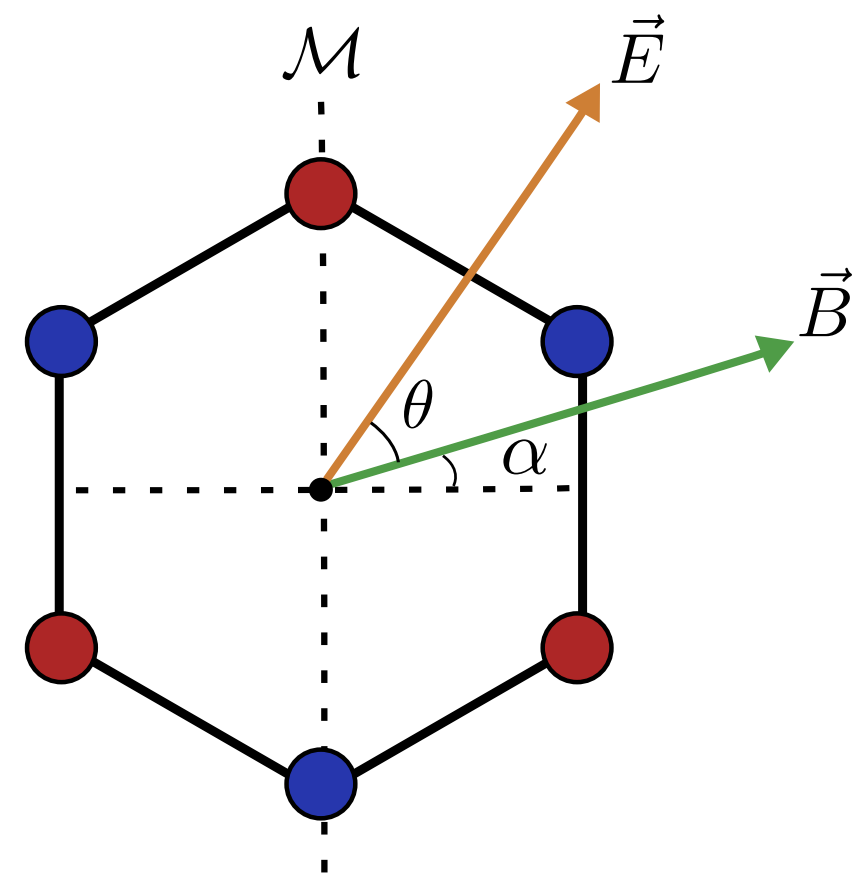

\begin{tabular}{|l|c|c|}
\hline \multirow{2}{*}{$\alpha=2 \pi n / 6$} & $\theta=0$ & Non-Linear APHE \\
\cline { 2 - 3 } & $\theta \neq 0$ & PHE \\
\hline \multirow{2}{*}{$\alpha \neq 2 \pi n / 6$} & $\theta=0$ & $\mathrm{APHE}$ \\
\cline { 2 - 3 } & $\theta \neq 0$ & $\mathrm{PHE}+\mathrm{APHE}$ \\
\hline
\end{tabular}

Figure 3.1: Schematic illustration of the conventional and anomalous PHEs in a two-dimensional trigonal crystal as a function of the relative angle $\theta$ between the electric $(\vec{E})$ and magnetic $(\vec{B})$ fields and the crystallographic angle $\alpha$ determined by $\vec{B}$ and the mirror line $\mathcal{M}$. 
crystal structures, as possible material platforms that can host both the linear and non-linear APHEs in the presence of Rashba spin-orbit coupling [9]. In these systems, the absence of inversion symmetry results in massive Dirac cones with a sizable Berry curvature. We find that the Berry curvatureinduced APHE vanishes only when the magnetic field is perpendicular to a mirror line of the trigonal crystal. In this situation, however, the finite Berry curvature dipole being still finite provides a non-linear anomalous PHE.

\subsection{Anomalous Planar Hall Effect}

Within the quasiclassical Boltzmann picture of transport, the transverse conductivity $\sigma_{y x}$ in the presence of coplanar electric and magnetic fields can be written as [60] [see also the Supplemental Material]:

$$
\begin{gathered}
\sigma_{y x}=e^{2} \int \frac{d^{3} k}{(2 \pi)^{3}} D \tau\left(-\frac{\partial f_{e q}}{\partial \epsilon}\right)\left\{\left[v_{y}+\frac{e B \sin \theta}{\hbar}\left(\mathbf{v}_{\mathbf{k}} \cdot \boldsymbol{\Omega}_{\mathbf{k}}\right)\right] .\right. \\
\left.\cdot\left[v_{x}+\frac{e B \cos \theta}{\hbar}\left(\mathbf{v}_{\mathbf{k}} \cdot \boldsymbol{\Omega}_{\mathbf{k}}\right)\right]\right\}+\frac{e^{2}}{h} \int \frac{d^{3} k}{\left(2 \pi^{3}\right)} \Omega_{k}^{z} f_{e q},
\end{gathered}
$$

where $f_{e q}$ is the equilibrium Fermi-Dirac distribution. In the equation above one can distinguish three different contributions: there is a first purely semiclassical term given by the weighted integral of the electronic velocities $v_{x} v_{y}$ that remains finite for strongly spin-orbit coupled and Zeeman spin-split electronic bands. This term is responsible for the PHE observed in (anti)ferromagnetic semiconductors and at oxide interfaces. The remaining contributions come about due to the anomalous velocity of Bloch electrons [83], and are therefore directly related to the Berry curvature $\boldsymbol{\Omega}(\mathbf{k})$. Specifically the terms containing the product $\boldsymbol{\Omega}(\mathbf{k}) \cdot \mathbf{v}(\mathbf{k})$ are responsible for the PHE in three-dimensional topological semimetals. Precisely as the classical contribution, the Berry curvature-induced PHE in Dirac and Weyl semimetals is even in the applied planar magnetic field, i.e. $\sigma_{x y}(B) \equiv \sigma_{x y}(-B)$ and hence does not satisfy the antisymmetry property of the conventional Hall conductivity. Finally, the last term given by the integral of the Berry curvature over the Fermi surface of the occupied states corresponds to the anomalous Hall effect characteristic of time-reversal broken materials that can be singled out by taking measurements at $B \equiv 0$. In two- 


\subsection{Anomalous Planar Hall Effect}

dimensional materials, since $\mathbf{v}_{\mathbf{k}} \perp \boldsymbol{\Omega}(\mathbf{k})$, the PHE is conventionally assumed to not possess any Berry curvature-induced contribution. Put differently the PHE of two-dimensional systems should not represent a "topological" response function. However, as we will show below, this conventional wisdom has to be re-evaluated in two-dimensional materials with a trigonal symmetry. The crux of the story is that the Zeeman spin splitting of the electronic bands induced by the planar magnetic field triggers a non-vanishing Berry curvature and thus engenders a planar Hall voltage that is entirely of quantum origin. In the linear response regime the consequence of this is twofold. First, this transverse conductance does obey the antisymmetry property of the conventional Hall conductance. Second, the transverse voltage is completely independent of the relative direction between the two coplanar fields. We dub this topological response anomalous planar Hall effect: it can be distinguished by the anomalous Hall effect by taking measurements at both $B \neq 0$ and $B \equiv 0$, and it can be singled out from the conventional PHE of two-dimensional systems by aligning the external magnetic and electric fields, or taking measurements at both $+B$ and $-B$.

As the Zeeman-induced Berry curvature obeys the symmetry properties of the crystal, point group symmetries can force the APHE response to vanish. Consider, for instance, a two-dimensional system subject to a planar magnetic field perpendicular to a mirror line of the crystal ( $\alpha=0$ in Fig. 3.1 ), which, without loss of generality, we assume to map a point with coordinates $\{x, y\}$ to $\{-x, y\}$. Since the external planar magnetic field preserves the mirror symmetry $\mathcal{M}_{x}$, the Berry curvature will obey the symmetry constraint $\Omega_{z}\left(k_{x}, k_{y}\right)=-\Omega_{z}\left(-k_{x}, k_{y}\right)$ even when the Zeeman spin splitting of the bands is fully taken into account. Furthermore, the Fermi surface must be symmetric with respect to the mirror line, and therefore the integral of the Berry curvature is forced to vanish. This, however, does not automatically imply the absence of a transverse planar Hall current when the driving electric field and the external magnetic field are collinear. The existence of a single residual mirror line still allows for a finite Berry curvature dipole defined by

$$
D_{b d}=\int_{k} f_{0}\left(\partial_{b} \Omega_{d}\right)
$$

which will be directed perpendicular to the residual mirror line and thus aligned with the magnetic and electric fields. In analogy with the quantum 
non-linear Hall effect in time-reversal symmetric conditions [18], a finite Berry curvature dipole causes a two-dimensional crystal subject to an AC driving electric field $E_{c}=\operatorname{Re}\left(\mathcal{E}_{c} e^{i \omega t}\right)$ to develop an additional non-linear current $j_{a}=\operatorname{Re}\left(j_{a}^{0}+j_{a}^{2 \omega} e^{2 i \omega t}\right)$ characterized by two Fourier components at zero and twice the frequency of the applied external field: $j_{a}^{0}=\chi_{a b c} \mathcal{E}_{b} \mathcal{E}_{c}^{*}$ and $j_{a}^{2 \omega}=\chi_{a b c} \mathcal{E}_{b} \mathcal{E}_{c}$. The response function $\chi_{a b c}$ has a "quantum" origin in the Berry curvature dipole and can be expressed as $\chi_{a b c}=-\epsilon_{a d c} e^{3} \tau D_{b d} / 2(1+$ $i \omega \tau), \epsilon_{a d c}$ being the Levi-Civita tensor and $\tau$ the scattering time. This quantum non-linear APHE coexists with a semiclassical second-order but Berry-phase independent contribution to the transverse non-linear Hall conductivity. The latter can be distinguished from the former since the Berry curvature dipole contributes to the antisymmetric dissipationless part of the non-linear Hall conductivity vector, defined as $\chi_{c}=\epsilon_{a b} \chi_{a b c} / 2$, while the semiclassical contribution is contained in the symmetric part of the response [84]. Finally, we emphasize that producing a non-vanishing dipole does not require a crystalline symmetry content as low as the one required in time-reversal symmetric conditions. This is because the externally applied planar magnetic field breaks all rotational and additional mirror symmetries thus partially relaxing the necessary conditions for a finite dipole. As a result, the non-linear Hall currents generated by the Berry curvature dipole vanish when the external magnetic field is set to zero, thus showing that this effect is a genuine Hall one.

\subsection{Symmetry analysis}

We now show that the (non)linear APHE naturally arises in strongly spinorbit coupled $2 \mathrm{D}$ crystals with $\mathcal{C}_{3 v}$ symmetry. First, we notice that a planar magnetic field is invariant under the combined $\mathcal{C}_{2} \mathcal{T}$ symmetry, where $\mathcal{C}_{2}$ indicates the twofold rotation around the axis perpendicular to the crystalline plane and $\mathcal{T}$ is the internal time-reversal symmetry. The presence of $\mathcal{C}_{2} \mathcal{T}$ symmetry then forces the Berry curvature to be identically zero: $\Omega_{z}(k) \equiv 0$. As a result, only trigonal crystals, which do not contain a twofold rotation symmetry, can display a planar magnetic-field induced non-trivial Berry curvature. Another necessary condition for the appearance of a finite Berry curvature dipole is the presence of a sizable spin-orbit coupling, which ensures that the crystal Hamiltonian $\mathcal{H}_{0}$ and the Zeeman coupling term $\mathcal{H}_{Z}=\vec{B} \cdot \vec{\sigma}$ 
do not commute. This prevents the possibility of separating the Bloch eigenfunctions of the full Hamiltonian $\mathcal{H}=\mathcal{H}_{0}+\mathcal{H}_{Z}$ into a spinorial part $\chi_{s}$, regulated only by the Zeeman term, and an orbital wavefunction $\psi_{\text {orb }}\left(k_{x}, k_{y}\right)$, where all the momentum dependence is stored: for eigenstates of that form the Berry curvature is indeed independent from the Zeeman coupling and retains the trigonal symmetry of the pristine crystal also in presence of the externally applied magnetic field. This forces the corresponding Berry curvature dipole to vanish. Finally, we notice that the non-linear PHE can occur only if the $\mathcal{S U}(2)$ spin symmetry in $\mathcal{H}_{0}$ is completely broken. A residual $\mathcal{U}(1)$ spin symmetry - as ensured by a mirror plane symmetry $\mathcal{M}_{z}$ - would in fact imply that $\mathcal{H}_{0}$ commutes with the spin rotation $\mathcal{U}_{\alpha}=e^{i \alpha \sigma_{z} / 2}$. This operator rotates the planar magnetic field by an angle $\alpha$ according to $\vec{B}^{\prime}=\mathcal{R}_{\alpha}(\vec{B})$, but since $\mathcal{U}_{\alpha}$ does not explicitly contain a momentum dependence, the two Hamiltonians $\mathcal{H}(\vec{B})$ and $\mathcal{H}^{\prime}=\mathcal{U}_{\alpha}^{\dagger} \mathcal{H}(\vec{B}) \mathcal{U}_{\alpha} \equiv \mathcal{H}\left(\vec{B}^{\prime}\right)$ have the same Berry curvature dipole. On the other hand, the dipole is forced to be parallel to the external magnetic field when the latter is orthogonal to a mirror line [85]. If we choose $\vec{B}$ and $\vec{B}^{\prime}$ to be perpendicular to different mirror lines (any two among the three of the $\mathcal{C}_{3 v}$ crystal), the only allowed vector compatible with such constraint is the null one. Hence, the Berry curvature dipole must vanish thereby proving that the non-linear planar Hall effect necessitates a complete breaking of the spin-rotation symmetry.

Having established the occurrence of a quantum non-linear PHE when the system is characterized by a residual mirror symmetry, we now consider the situation in which the external planar magnetic field is not constrained to be orthogonal to one of the three mirror lines of the $\mathcal{C}_{3 v}$ crystal. Since the presence of the planar magnetic field reduces the point group to the trivial group $\mathcal{C}_{1}$, the Berry curvature does not obey any constraint, and therefore the net anomalous velocity is not forced to vanish. This consequently leads to the possibility of a purely Zeeman-induced quantum PHE in the linear response regime, which represents an antisymmetric contribution to the resistivity tensor and therefore displays a $2 \pi$ periodic angular dependence. Furthermore, it is important to notice that for the integral of the Berry curvature weighed by the equilibrium Fermi distribution function to be non zero the spin-rotation symmetry needs to be completely broken - in a crystal with a $\mathcal{M}_{z}$ mirror plane, the combined $\mathcal{M}_{z} \mathcal{T}$ symmetry, which is still preserved with a planar magnetic field, forces the Berry curvature to be an odd function. Hence, as for its non-linear counterpart, also the quantum PHE 


\section{Anomalous Planar Hall Effect in Two-Dimensional Trigonal Crystals}

in linear response can only occur in strongly spin-orbit coupled crystals. It is thus expected to coexist with the conventional Berry-phase independent contribution to the PHE, which, as stated above, represents instead a symmetric part of the resistivity tensor. These different symmetry properties of the quantum and semiclassical contributions to the linear PHE imply that the "semiclassical" linear contribution to the PHE can be isolated in experiments by taking measurements with both positive and negative B. Instead, since the quantum contribution is independent of the angle between the electric and magnetic field, in a configuration where they are parallel it is the only term that survives.

\subsection{Model}

Next, we show that monolayer graphene with a (substrate-induced) inversion symmetry breaking mass, as well as TMDs in their trigonal structure support the existence of both the APHE and the non-linear APHE in the presence of Rashba spin-orbit coupling. To show this, we consider a general microscopic tight-binding model featuring massive Dirac cones on the honeycomb lattice

$$
\begin{aligned}
\mathcal{H}_{c r y}= & -t \sum_{i=1}^{3}\left[\cos \left(\mathbf{k} \cdot \boldsymbol{\delta}_{\boldsymbol{i}}\right) \tau_{x}+\sin \left(\mathbf{k} \cdot \boldsymbol{\delta}_{\boldsymbol{i}}\right) \tau_{y}\right] \otimes \sigma_{0}+ \\
& \frac{\Delta}{2} \tau_{z} \otimes \sigma_{0}+\mathcal{H}_{R},
\end{aligned}
$$

where $\sigma$ and $\tau$ refer to the spin and sublattice degrees of freedom respectively, and $\left\{\boldsymbol{\delta}_{1}, \boldsymbol{\delta}_{2}, \boldsymbol{\delta}_{3}\right\}=\{(0, a / \sqrt{3}),(a / 2,-a / 2 \sqrt{3}),(-a / 2,-a / 2 \sqrt{3})\}$ are the nearest neighbours with $a$ the honeycomb lattice constant. In the Hamiltonian of Eq. 3.3 the first term containing nearest neighbour spin-independent hopping respects the $\mathcal{C}_{6 v}$ point group symmetry of the honeycomb lattice, which is generated by the three-fold rotation symmetry $\mathcal{C}_{3 v}=\tau_{x} \otimes e^{i \pi \sigma_{z} / 6}$, the twofold rotation symmetry $\mathcal{C}_{2}=\tau_{x} \otimes e^{i \pi \sigma_{z} / 2}$, and the mirror symmetry $\mathcal{M}_{x}=\tau_{0} \otimes e^{i \pi \sigma_{x} / 2}$. In order to reduce the crystalline symmetry to be trigonal, we have introduced the $\mathcal{C}_{2}$ and inversion-symmetry breaking mass $\propto \Delta$. In graphene, the latter term is naturally realized by placing the graphene flake on lattice-matched substrates, such as hexagonal boron 


\subsection{Model}
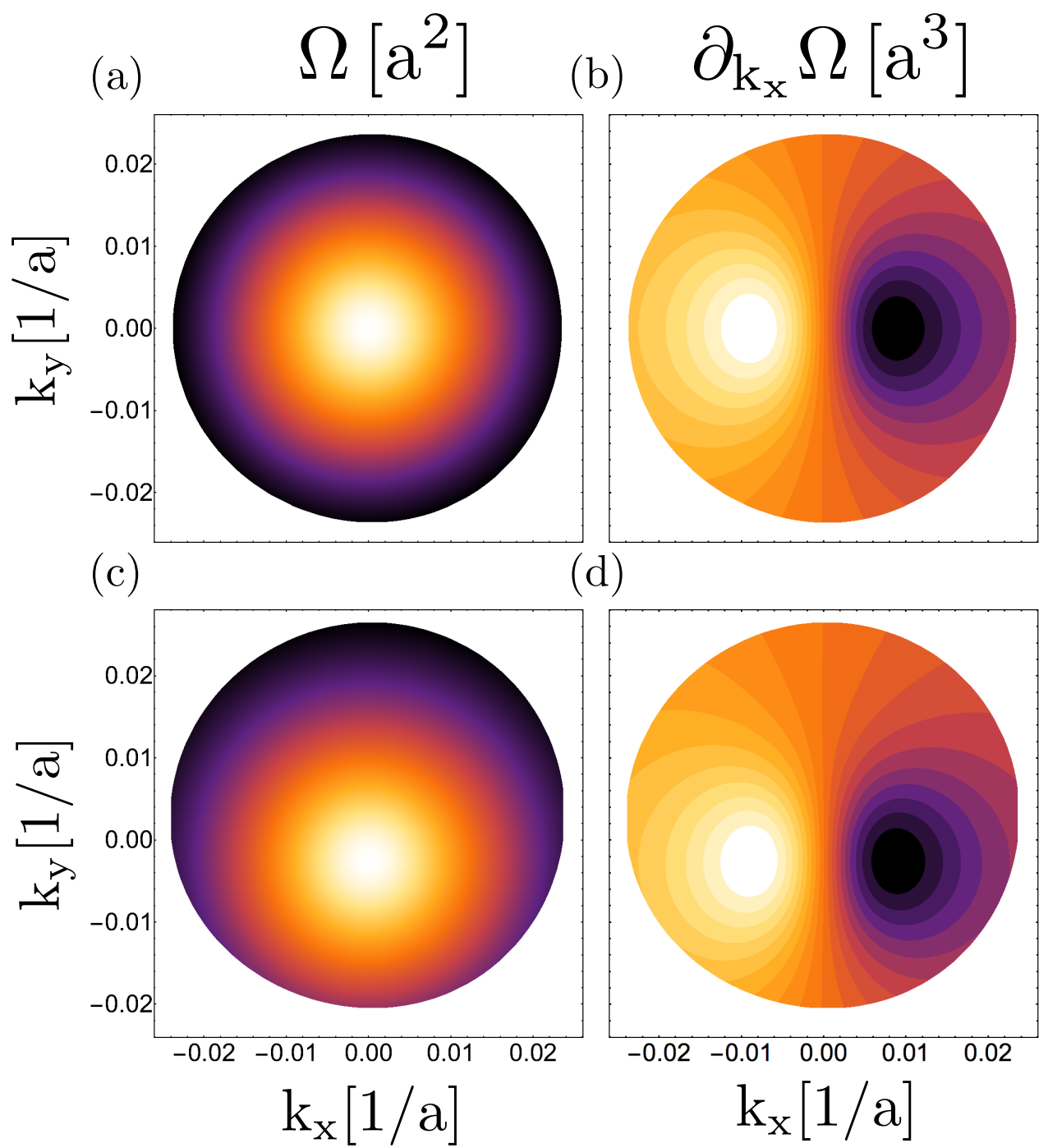

Figure 3.2: Berry curvature $\Omega(\mathrm{a}, \mathrm{c})$ and dipole density $\partial_{k_{x}} \Omega(\mathrm{b}, \mathrm{d})$ of the conduction bands corresponding to the Hamiltonian of Eq. 3.3 in the absence $(\mathrm{a}, \mathrm{b})$ and presence $(\mathrm{c}, \mathrm{d})$ of Rashba spin-orbit coupling $\left(\lambda_{R} / t=10^{-2}\right)$. The magnetic field $\left(B / t=10^{-3}\right)$ has been placed along the zig-zag direction, $\alpha=0$, preserving the mirror symmetry $\mathcal{M}_{x}$. The two valleys at $K$ and $K^{\prime}$ are related by $\mathcal{M}_{x}$ and hence contribute identically to the Berry curvature dipole. The inversion breaking mass has been taken to be $\Delta / t=5 \times 10^{-2}$. In plots (b) and (d) light colors correspond to positive values while darker colors correspond to negative ones. 

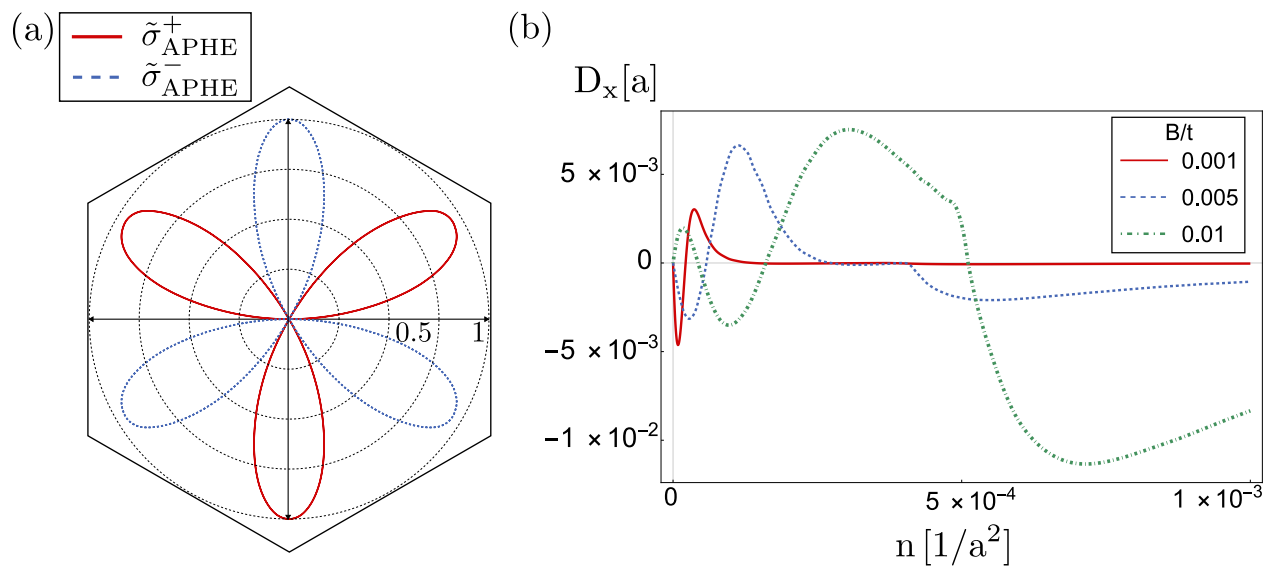

Figure 3.3: (a) Polar plot of the anomalous planar Hall conductivity as a function of the angle $\alpha$ between the planar magnetic field and the zig-zag direction of the honeycomb lattice. The value of $\sigma_{\mathrm{PHE}}$ is normalized to the maximum at $\alpha=\pi / 2$. The conductivities $\tilde{\sigma}_{\mathrm{APHE}}^{ \pm}$represent, respectively, the positive and negative part of the full APHE conductivity and are normalized to the maximum $\alpha=\pi / 2$. Parameters used for the plot are: $\Delta / t=5 \times 10^{-2}, B / t=10^{-2}, \lambda_{R} / t=10^{-2}$. (b) Berry curvature dipole $\mathrm{D}_{\mathrm{x}}$ as a function of the carrier density. Parameters used for the plot are: $\Delta / t=5 \times 10^{-2}, \alpha=0, B / t=10^{-3}$, $\lambda_{R} / t=10^{-2}$.

nitride [41, 42]. Finally, the last term in Eq. 3.3 is a Rashba-like spin-orbit coupling term that fully breaks the $\mathcal{S U}(2)$ spin symmetry and therefore allows for a non-vanishing Berry curvature dipole when an external planar magnetic field is applied. The Rashba term [86] can be written as $\mathcal{H}_{R}=$ $\sqrt{3} \lambda_{R} \sum_{i=1}^{3}\left[\sin \left(\mathbf{k} \cdot \boldsymbol{\delta}_{i}\right) \tau_{x} \otimes\left(\sigma_{y} \boldsymbol{\delta}_{i, 1}-\sigma_{x} \boldsymbol{\delta}_{i, 2}\right)+\cos \left(\mathbf{k} \cdot \boldsymbol{\delta}_{i}\right) \tau_{y} \otimes\left(\sigma_{y} \boldsymbol{\delta}_{i, 1}-\sigma_{x} \boldsymbol{\delta}_{i, 2}\right)\right]$, with the strength of the Rashba coupling $\lambda_{R}$ that in graphene is controlled by the strength of the perpendicular electric field, and the local curvature of the graphene sheet [87].

We finally account for the external planar magnetic field introducing the Zeeman coupling term $\mathcal{H}_{Z}=B \tau_{0} \otimes\left(\sigma_{x} \cos \alpha+\sigma_{y} \sin \alpha\right)$ where $\alpha$ is the angle from the zig-zag direction of the honeycomb lattice. For $\alpha=2 n \pi / 6$ with $n \in \mathbb{N}$ the magnetic field preserves one mirror symmetry thus allowing only for a Berry curvature dipole. In the absence of spin-orbit interaction, i.e. for $\lambda_{R} \equiv 0$, the Zeeman coupling leads to a closing of the half-filling gap at 


\subsection{Model}

the critical strength $B_{c} \equiv \Delta / 2$, above which the system becomes a nodal semimetal generated by the crossing of two bands belonging to different spin sectors. A finite value of the Rashba spin-orbit coupling changes the crossings into anticrossings, and thus the system has a finite half-filling gap as long as the strength of the applied magnetic field is of the same order of magnitude as the inversion-symmetry breaking mass $\Delta$. For larger values of the applied magnetic field $B \simeq 2 \Delta$, the half-filling gap closes but we will neglect this regime in the remainder.

More importantly, a finite value of $\lambda_{R}$ changes the distribution of the Berry curvature allowing for a non-zero Berry curvature dipole. This is explicitly demonstrated in Fig. 3.2 where we show the local Berry curvature, computed using the method outlined in Ref. [88], both in the absence and in the presence of the Rashba spin-orbit interaction. We find that effect of the Rashba spin-orbit coupling is twofold. First, it boosts the Berry curvature by reducing the splitting between the two conduction and valence bands. Second, it shifts the dipole distribution away from being centred around the high symmetry points $K$ and $K^{\prime}$, hence allowing for an overall finite dipole. Fig. 3.3b shows the behavior of the ensuing Berry curvature dipole as a function of the carrier density for various values of the external planar magnetic field. We generally find that increasing the external magnetic field strength boosts the amplitude of the dipole over a larger range of carrier density. The dipole also displays a characteristic non-monotonous behavior, similar to the one theoretically predicted and experimentally observed [31, 32, 35] in the time-reversal non-linear Hall effect, with various sign reversals, which implies that the quantum contribution to the transverse current changes direction. We note that a similar non-monotonous behavior is also found in the semiclassical symmetric contribution to the non-linear Hall conductance as shown in the Supplemental Material.

Finally, we have computed the linear quantum contribution to the PHE for $\alpha \neq 2 \pi n / 6$. As shown in Fig. 3.3a, we find that the integral of the Berry curvature weighed by the equilibrium Fermi distribution contributes to the PHE with an angular dependence that only depends on the relative direction between the magnetic field and the principal crystallographic direction, and changes sign under a $\pi$ rotation of the planar magnetic field, in perfect agreement with our general analysis. This dependence is different than the semiclassical contribution $\sigma_{x y}=e^{2} \tau \int_{k} v_{x} v_{y}\left(-\partial f_{0} / \partial \varepsilon_{k}\right)$ [89], which we find to depend exclusively on the angle between the coplanar electric and 
magnetic field [see the Supplemental Material] and follows the usual PHE $\cos \theta \sin \theta$ behavior, thus vanishing when the applied fields are aligned.

\subsection{Conclusions}

In short, we have shown that two-dimensional trigonal crystals with sizable spin-orbit coupling subject to planar magnetic fields display a previously unknown planar Hall effect that contributes to the dissipationless Hall conductance. We dubbed this contribution anomalous planar Hall effect. This effect is rooted in the geometric properties of the Bloch states encoded in the Berry curvature and appears whenever the planar magnetic field does not leave any residual mirror line. It can be effectively decoupled from the conventional PHE since it survives even when the driving electric field and the planar magnetic field are aligned. Moreover we have found that in a configuration in which the coplanar fields are aligned and perpendicular to one of the mirror lines of the crystal, transverse Hall currents still exist and appear at second order in the driving electric field. The resulting non-linear anomalous planar Hall effect has a quantum origin arising from the first moment of the Berry curvature, the Berry curvature dipole. Finally, we propose monolayer graphene on commensurate hexagonal boron nitride substrates as well as transition metal dichalcognides with trigonal structure as possible material platform where the (non-)linear APHE can be experimentally observed. 


\section{A Appendix}

\section{A.1 Linear Planar Hall Effects in 2D and 3D}

In this section we discuss the difference between the planar Hall effect in twoand three-dimensional systems using a semiclassical Boltzmann framework. We assume to apply an electric field $\mathbf{E}=E \hat{\mathbf{x}}$ along the $\hat{x}$ axis and rotate the magnetic field in the $x-y$ plane. Thus we write the magnetic field as $\mathbf{B}=B(\cos \theta \hat{\mathbf{x}}+\sin \theta \hat{\mathbf{y}})$. The semiclassical equations of motions in the presence of a non-zero Berry curvature read

$$
\begin{aligned}
& \dot{\mathbf{r}}=D\left(\mathbf{B}, \boldsymbol{\Omega}_{\mathbf{k}}\right)\left[\mathbf{v}_{\mathbf{k}}+\frac{e}{\hbar}\left(\mathbf{E} \times \boldsymbol{\Omega}_{\mathbf{k}}\right)+\frac{e}{\hbar}\left(\mathbf{v}_{\mathbf{k}} \cdot \boldsymbol{\Omega}_{\mathbf{k}}\right) \mathbf{B}\right] \\
& \hbar \dot{\mathbf{k}}=D\left(\mathbf{B}, \boldsymbol{\Omega}_{\mathbf{k}}\right)\left[e \mathbf{E}+\frac{e}{\hbar}\left(\mathbf{v}_{\mathbf{k}} \times \mathbf{B}\right)+\frac{e^{2}}{\hbar}(\mathbf{E} \cdot \mathbf{B}) \boldsymbol{\Omega}_{\mathbf{k}}\right]
\end{aligned}
$$

where $D\left(\mathbf{B}, \boldsymbol{\Omega}_{\mathbf{k}}\right)=\left[1+(e / \hbar)\left(\mathbf{B} \cdot \boldsymbol{\Omega}_{\mathbf{k}}\right)\right]^{-1}, \boldsymbol{\Omega}_{\mathbf{k}}$ is the Berry curvature and $\mathbf{v}_{\mathbf{k}}$ is the group velocity. Solving the Boltzmann equation for the electron distribution function $f(k)$ within the relaxation time approximation allows to compute the charge current $\mathbf{J}=e \int\left(d^{D} k /(2 \pi)^{D}\right) \dot{\mathbf{r}} f(k) D^{-1}$ that accounts for the modified phase space factor $D$. In linear response theory the charge current obeys the relation $J_{a}=\sigma_{a b} E_{b}$, where $\sigma_{a b}$ are the components of the conductivity tensor and $E_{b}$ the external electric field. Following Ref. [60], the planar Hall conductivity, after discarding higher order contributions, is given by

$$
\begin{gathered}
\sigma_{y x}=e^{2} \int \frac{d^{3} k}{(2 \pi)^{3}} D \tau\left(-\frac{\partial f_{e q}}{\partial \epsilon}\right)\left\{\left[v_{y}+\frac{e B \sin \theta}{\hbar}\left(\mathbf{v}_{\mathbf{k}} \cdot \boldsymbol{\Omega}_{\mathbf{k}}\right)\right] .\right. \\
\left.\cdot\left[v_{x}+\frac{e B \cos \theta}{\hbar}\left(\mathbf{v}_{\mathbf{k}} \cdot \boldsymbol{\Omega}_{\mathbf{k}}\right)\right]\right\}+\frac{e^{2}}{h} \int \frac{d^{3} k}{\left(2 \pi^{3}\right)} \Omega_{k}^{z} f_{e q},
\end{gathered}
$$

where $f_{e q}$ is the equilibrium Fermi-Dirac distribution. Eq. 3.6 contains all transverse linear responses in the presence of coplanar electric and magnetic fields in both two- and three-dimensional systems. In three-dimensional topological Dirac and type-I Weyl semimetals, the Berry-curvature induced planar Hall effect stems from the term $\propto B^{2} \sin \theta \cos \theta\left(\mathbf{v}_{\mathbf{k}} \cdot \boldsymbol{\Omega}_{\mathbf{k}}\right)^{2}$. The last term instead represents the usual anomalous Hall contribution that can be 


\section{Anomalous Planar Hall Effect in Two-Dimensional Trigonal Crystals}

singled out by taking measurements at $B=0$.

In two-dimensional systems, $\mathbf{v}_{\mathbf{k}} \perp \boldsymbol{\Omega}_{\mathbf{k}}$, that would therefore imply the generic absence of any Berry-curvature induced planar Hall effect in nonmagnetic systems. As shown in the main part of the manuscript, however, in two-dimensional trigonal crystals a non-vanishing contribution to the last term of Eq. 3.6 appears once the Zeeman interaction is explicitly taken into account, and exists even though the anomalous Hall effect vanishes. This also implies that, contrary to three-dimensional topological semimetals, the Berry-curvature related quantum planar Hall effect of two-dimensional systems does not explicitly depend on the relative angle between the electric and magnetic field but only on the angle between the planar magnetic field and the principal crystallographic directions. This, in turns, allows to directly observe the quantum planar Hall effect when the electric and magnetic fields are perfectly aligned since in this configuration the classical contribution of Eq. 3.6, containing the $v_{x} v_{y}$ term, vanishes as discussed in the main part of the manuscript.

\section{A.2 Phase Behavior of the Model Hamiltonian}

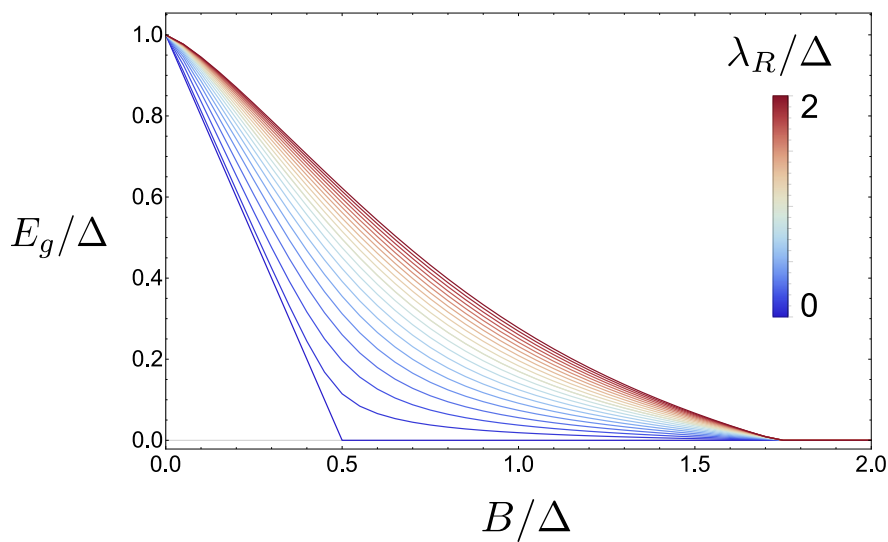

Figure 3.4: Phase behavior of the energy gap of the model Hamiltonian as a function of the strength of the Rashba spin-orbit coupling and the magnetic field.

The energy gap of the Hamiltonian introduced in Section 3 of the main manuscript can be closed depending on the relative strength between the 


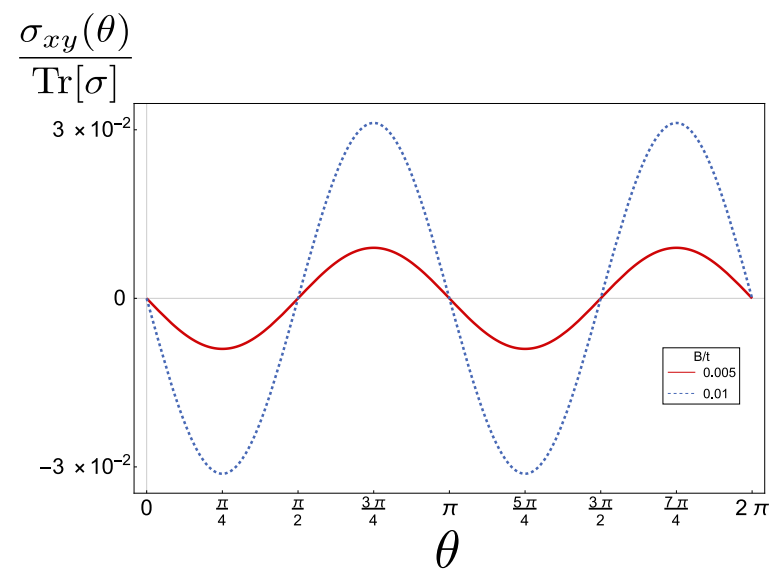

Figure 3.5: $\sigma_{x y}$ of Eq. 3.7 as a function of the relative angle $\theta$ between the applied electric and magnetic fields. Parameters used for the plot are: $\Delta / t=$ $5 \times 10^{-2}$ and $\lambda_{R} / t=10^{-2}$. The conductivity is normalized to the invariant trace $\operatorname{Tr}[\sigma]=\sigma_{x x}(\theta)+\sigma_{y y}(\theta)$ of the conductivity tensor.

Rashba coupling $\lambda_{R}$ and the Zeeman strength $B$. As shown in Fig. 3.4 at zero Rashba the magnetic field closes the gap at the critical value $B_{c}=\Delta$. Adding the Rashba coupling reopens the gap since band crossings become anti-crossings. For sufficiently strong magnetic fields however the gap can once again be closed. By tuning the magnetic field it is thus possible to tune the strength of the Berry curvature and the its first moment.

\section{A.3 Semiclassical Linear Planar Hall Effect}

The semiclassical contribution to the linear planar Hall currents is given by,

$$
\sigma_{x y}=e^{2} \tau \int_{k} v_{x} v_{y}\left(-\partial f_{0} / \partial \varepsilon_{k}\right)
$$

where $v_{i}=\partial_{i} \varepsilon_{i}$ with $i=(x, y), \tau$ is the relaxation time and $f_{0}$ the equilibrium Fermi-Dirac distribution. As can be seen in Fig. 3.5 this contribution is $\pi$ periodic and doesn't flip sign when the magnetic field changes sign. Furthermore the $\sigma_{x y}$ vanishes when the magnetic and electric field are collinear or perpendicular. 


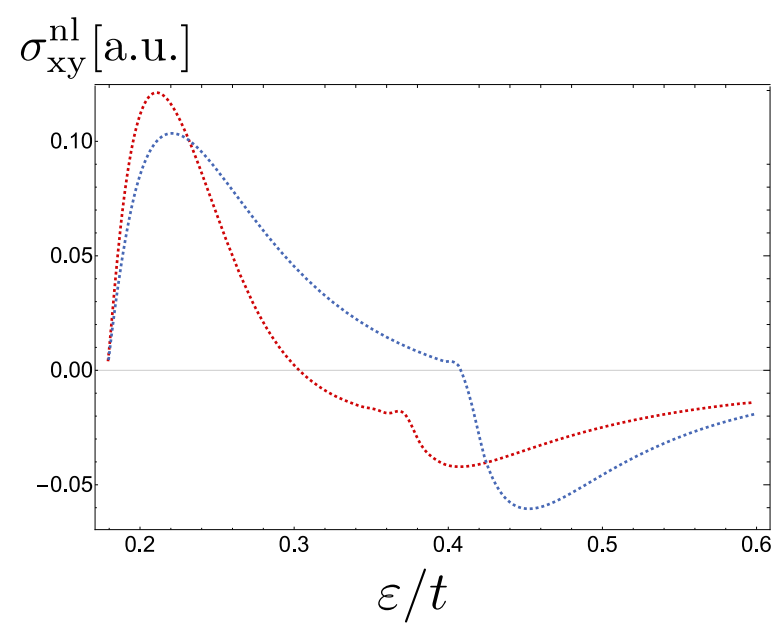

Figure 3.6: Non-linear aniostropic magnetoresistance as a function of the Fermi energy. Parameters used for the plot are: $\Delta / t=5 \times 10^{-2}, \lambda_{R} / t=$ $10^{-2}, B / t=5 \times 10^{-3}$ in (red) and $B / t=10^{-2}$ in (blue).

\section{A.4 Semiclassical Non-Linear Planar Hall Effect}

The semiclassical contributions to the non-linear planar Hall current are given by the second terms of Eq. 1.38. Assuming a magnetic field along the zig-zag direction the non-linear transverse current then only runs along the armchair and is maximized when electric and magnetic field are collinear. In this case assuming the zig-zag is along the $\mathrm{x}$ coordinate, the non-linear contribution to the transverse conductivity is given by:

$$
\sigma_{\mathrm{xy}}^{\mathrm{nl}}=\frac{e^{3} \tau^{2}}{2(1+i \omega \tau)} \int_{k} \partial_{y} \varepsilon_{k} \partial_{x}^{2} f_{0}
$$

This contribution scales like $\tau^{2}$ in the DC limit of $\omega \rightarrow 0$ whereas for $\omega \tau>>1$ it scales like $\tau$. The dipole contribution of Eq. 1.38 on the other hand scales like $\tau$ in the former case and becomes scattering independent for the latter case. Eq. 3.8 is plotted in Fig. 3.6 as a function of the Fermi energy. The kinks in the non-linear conductivity occur when the second conduction band starts being filled. 


\section{Anomalous Planar Hall Effect in a Rashba Two-Dimensional Electron Gas}

Two-dimensional electron gasses (2DEG) with Rashba spin-orbit coupling (SOC) [9] are found in a variety of non-centrosymmetric materials. The spinmomentum locking caused by the Rashba SOC is a key ingredient in many fields of physics and materials science, ranging from the spin-Hall effect all the way to Majorana Fermions [10]. In connection with Chapter 3 we discuss the occurrence of an anomalous planar Hall effect (APHE) in a Rashba 2DEG. We consider the minimal two band Hamiltonian and show that the Berry curvature stems from higher order momentum terms that are invariant under threefold rotations. This simple model shares the same symmetries of the honeycomb model discussed in Chapter 3 with the main difference that the Rashba 2DEG describes the low energy properties of materials around the high symmetry point $\Gamma$ and is host to a very different kind of physics compared to a Dirac cone. The occurrence of the anomalous planar Hall effect (APHE) in a conventional Rashba 2DEG widens the horizon of material candidates in which the effect can be observed.

\subsection{Model Hamiltonian}

The minimal Hamiltonian describing a Rashba 2DEG is typically an effective two-band model derived using $\mathbf{k} \cdot \mathbf{p}$ theory around the high symmetry point $\Gamma$. We write the Hamiltonian in the basis of a spin- $1 / 2$ electron $\left|\psi^{\uparrow \downarrow}\right\rangle$ such that the anti-unitary time reversal operator has the representation $\mathcal{T}=i \sigma_{y} \mathcal{K}$ while the mirror symmetry is $\mathcal{M}=i \sigma_{x}$. At linear order in the momentum k the Rashba Hamiltonian reads,

$$
\mathcal{H}_{R}(\mathbf{k})=\frac{\mathbf{k}^{2}}{2 m}-\alpha_{R} \boldsymbol{\sigma} \cdot \mathbf{k} \times \hat{\mathbf{z}}
$$

where $\boldsymbol{\sigma}$ is a vector of Pauli matrices, $\alpha_{R}$ is the Rashba coupling strength and $m$ is the electronic mass. The Hamiltonian in Eq. 4.1 preserves time- 


\section{Anomalous Planar Hall Effect in a Rashba Two-Dimensional Electron Gas}

reversal symmetry and is rotationally invariant. However, the Berry curvature associated to this minimal model is simply zero. As discussed in Chapter 3 the anomalous planar Hall effect can only appear in trigonal crystals without $\mathcal{C}_{2} \mathcal{T}$ symmetry. Moreover, effective theories can also account for warping effects which comply with the underlying crystalline symmetries. For this reason one can include higher order momentum terms which make the model $\mathcal{C}_{3}$ symmetric. In the basis $\left|\psi^{\uparrow \downarrow}\right\rangle$ the threefold rotation operator takes the form $\mathcal{C}_{3}=e^{-i \sigma_{z} \pi / 3}$. Under the operation of $\mathcal{C}_{3}$ and $\mathcal{M}$, momentum and spin transform as follows,

$$
\begin{aligned}
& \mathcal{C}_{3}: \quad k_{ \pm} \rightarrow e^{ \pm i 2 \pi / 3} k_{ \pm}, \quad \sigma_{ \pm} \rightarrow e^{ \pm i 2 \pi / 3} \sigma_{ \pm}, \quad \sigma_{z} \rightarrow \sigma_{z} \\
& \mathcal{M}: \quad k_{+} \rightarrow-k_{-} \quad \sigma_{x} \rightarrow \sigma_{x}, \quad \sigma_{y, z} \rightarrow-\sigma_{y, z}
\end{aligned}
$$

where $k_{ \pm}=k_{x} \pm i k_{y}$ and $\sigma_{ \pm}=\sigma_{x} \pm i \sigma_{y}$. The Hamiltonian must also be invariant under time reversal which adds the constraint $\mathcal{H}(\mathbf{k})=\mathcal{T} \mathcal{H}(-\mathbf{k}) \mathcal{T}^{-1}=$ $\sigma_{y} \mathcal{H}^{*}(-\mathbf{k}) \sigma_{y}$. The first symmetry allowed term is third order in momentum and takes the form,

$$
\mathcal{H}_{w}(\mathbf{k})=\frac{\lambda}{2}\left(k_{+}^{3}+k_{-}^{3}\right) \sigma_{z}
$$

The warping Hamiltonian of Eq. 4.3 is proportional to the Pauli matrix $\sigma_{z}$, which is crucial to obtain a non-zero Berry curvature. It is also important to note that since the full Hamiltonian is invariant under the mirror symmetry $\mathcal{M}, \mathcal{H}_{w}(\mathbf{k})$ is forced to vanish along the mirror line. The eigenvalues of $\mathcal{H}=\mathcal{H}_{R}+\mathcal{H}_{w}$ in polar coordinates are given by,

$$
\varepsilon_{ \pm}(k, \theta)=\frac{k^{2}}{2 m} \pm \sqrt{\alpha_{R}^{2} k^{2}+\lambda^{2} k^{6} \cos ^{2}(3 \theta)}
$$

The Rashba coupling lifts the spin-degeneracy and splits the two bands (Fig. 4.2a). At $k=0$ a Kramer's degeneracy is present. The two bands $\epsilon_{ \pm}$display opposite spin textures (one moving clockwise, the other counterclockwise), this can be seen by taking the expectation values of $\langle\boldsymbol{\sigma}\rangle_{ \pm}$(the behaviour is qualitatively the same as the one shown in Fig. 4.1). From Eq. 4.3 we see that the Fermi surfaces displays an hexagonal warping: this is due to the combined presence of $\mathcal{C}_{3}$ and $\mathcal{T}$ symmetry. This hexagonal 


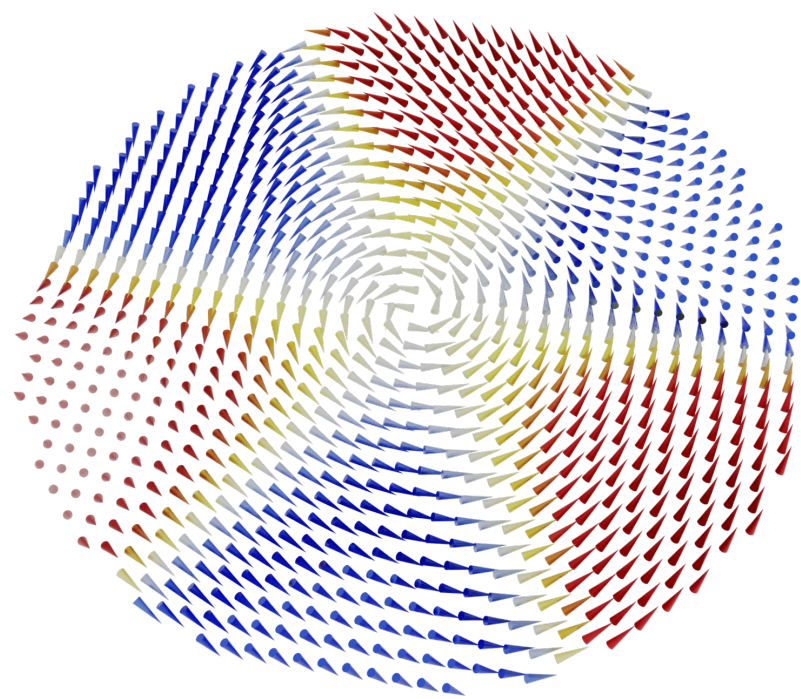

Figure 4.1: Texture of the normalized $\mathbf{d}$-vector in momentum space.

warping effect has been observed in the surface states of $\mathrm{Bi}_{2} \mathrm{Te}_{3}$ topological insulators and a similar Hamiltonian has been used to explain the ARPES measurements [90]. It is important to note however, that in this case the Hamiltonian was used in a different parameter range allowing for the description of the anomalous Dirac cones present on the surface of 3DTIs. We are interested in what happens to the Berry curvature when considering such a threefold symmetric term. Remarkably, by adding the warping term a nontrivial Berry curvature emerges for the Rashba 2DEG. In a two band model the Berry curvature can be simply calculated by rewriting the Hamiltonian using the $\mathbf{d}$-vector, such that $\mathcal{H}(\mathbf{k})=\mathbf{d}(\mathbf{k}) \cdot \boldsymbol{\sigma}$. We can then use the fact that $\Omega_{z}^{ \pm}(\mathbf{k})= \pm \hat{\mathbf{d}} \cdot\left(\partial_{k_{x}} \hat{\mathbf{d}} \times \partial_{k_{y}} \hat{\mathbf{d}}\right) / 2$ to find,

$$
\Omega_{ \pm}^{z}(k, \theta)= \pm \frac{2 \sqrt{2} \lambda \cos (3 \theta) \alpha_{R}^{2}}{\left(\lambda^{2} k^{4}(\cos (6 \theta)+1)+2 \alpha_{R}^{2}\right)^{3 / 2}}
$$

The non-trivial behaviour of the $\mathbf{d}$-vector in momentum space is shown in Fig. 4.1. The Berry curvature is well defined in each point except the origin where the bands are degenerate and the abelian description breaks 
4 Anomalous Planar Hall Effect in a Rashba Two-Dimensional Electron Gas
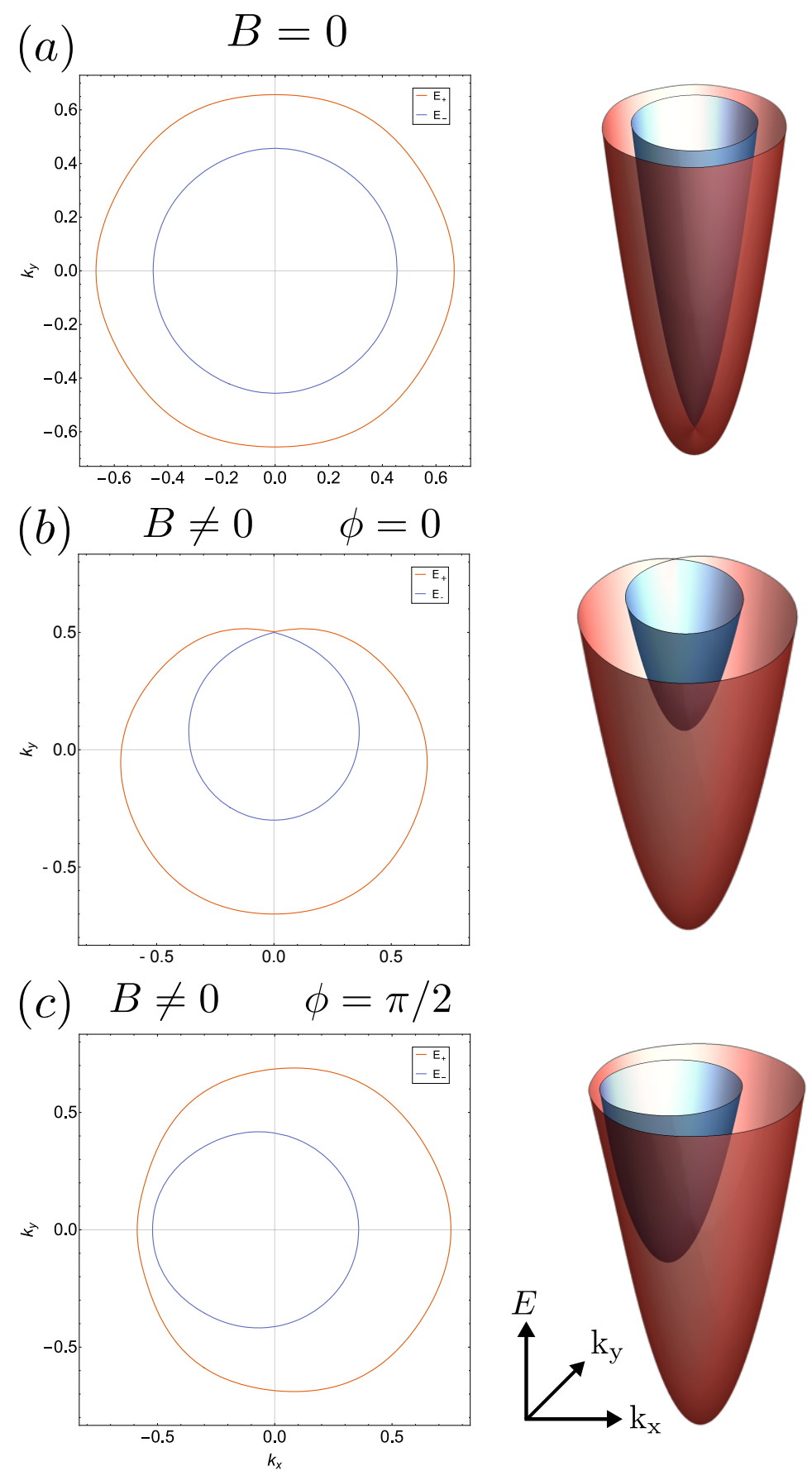

Figure 4.2: Energy contours and 3D dispersions of the warped Rashba Hamilto56 nian with Zeeman coupling. 
down. The constraints set by time reversal symmetry and the three-fold rotational symmetry are satisfied as can be verified upon a closer inspection of Eq. 4.4. Moreover $\Omega_{ \pm}^{z}(k, \theta)$ vanishes along the mirror lines, in accordance with Eq. 4.2. Unfortunately, observing the Berry curvature of such a system is a nearly impossible task since the two bands contribute to exactly opposite curvatures (Fig. 4.3a). Since the largest source of Berry curvature is found in the vicinities of the degeneracy point, for Fermi energies that cross both bands (which is typically what is found for experimental density regimes) it is reasonable to expect a complete suppression of any type of Berry curvature effect due to the exact cancellation between the two bands. This is no longer the case when applying a planar magnetic field. Indeed, such a magnetic field generates a Zeeman coupling term $\mathcal{H}_{B}=B\left(\sigma_{x} \cos \phi+\sigma_{y} \sin \phi\right)$, that breaks $\mathcal{C}_{3}, \mathcal{T}$ and the remaining mirror $\mathcal{M}_{x}$. For $\phi=2 \pi m / 6$ with $m \in \mathbb{N}$, one mirror symmetry is preserved. The energy dispersion and Berry curvature of the Zeeman-split Hamiltonian are given by,

$$
\begin{aligned}
\varepsilon_{ \pm}^{B}(k, \theta) & =\frac{k^{2}}{2 m} \pm \\
& \sqrt{\frac{2 B^{2}+2 \alpha_{R} k\left(2 B \sin (\phi-\theta) \alpha_{R} k\right)+\lambda^{2} k^{6} \cos (6 \theta)+\lambda^{2} k^{6}}{2}} \\
\Omega_{ \pm}^{z, B}(k, \theta) & = \\
& \pm \frac{\sqrt{2} \lambda \alpha_{R} k^{2}\left(3 B \sin (2 \theta+\phi)+2 \alpha_{R} k \cos (3 \theta)\right)}{\left(2 B^{2}+2 \alpha_{R} k\left(2 B \sin (\phi-\theta)+\alpha_{R} k\right)+\lambda^{2} k^{6} \cos (6 \theta)+\lambda^{2} k^{6}\right)^{3 / 2}}
\end{aligned}
$$

In general, as shown in Fig. 4.2c, adding a magnetic field breaks the Kramer's degeneracy and completely splits the two bands. However, when a mirror is preserved so is the degeneracy point (Fig. 4.2b), which shifts from the origin along the mirror line at $(k, \theta)=\left(|B| / \alpha_{R}, \phi+\operatorname{sgn}(B) \pi / 2\right)$. By varying the Fermi energy it is then possible to probe this source of Berry curvature since it is no longer confined at $k=0$ (Fig. 4.3b). Moreover, a Lifschitz transition (a change in topology in the Fermi surface, in this case going from one closed Fermi line to two) appears since the Zeeman coupling shifts the minima of the two bands (Fig. 4.2b,c). The energy for which the 


\section{Anomalous Planar Hall Effect in a Rashba Two-Dimensional Electron Gas}

upper band is completely depleted is given by $\varepsilon_{L}=B^{2} /\left(2 m \alpha_{R}^{2}\right)$ if $B<m \alpha_{R}^{2}$ otherwise $\varepsilon_{L}=B-m \alpha_{R}^{2} / 2$ if $B>m \alpha_{R}^{2}$.

\subsection{Anomalous Planar Hall Effect}

Given that the Zeeman splitting satisfies all the symmetry constraints necessary for a finite APHE we can compute the APHE conductance,

$$
\sigma_{\mathrm{APHE}}=\sum_{n= \pm} \int_{\mathbf{k}} \frac{d^{2} k}{(2 \pi)^{2}} \Omega_{n}^{z, B}(\mathbf{k}) f_{0}\left(\varepsilon_{n}^{B}(\mathbf{k})\right)
$$

As shown in Fig. 4.4a the angular dependence of the APHE follows the three-fold symmetry of the underlying model in a similar manner to what was shown in Chapter 3.

The magnetic field dependence at fixed density shown in Fig. 4.4b can be understood by considering how the Fermi energy of the system is modified when varying $B$. At zero field, the Fermi energy is taken to occupy both spin bands. We notice that by sufficiently increasing $B$ the conductance starts to increase, this occurs when the field dependent Fermi energy (which has to vary in order to keep the carrier density fixed) starts approaching the band touching point where the peak of the $\sigma_{\mathrm{APHE}}$ occurs. Further increasing the magnetic field leads to a decline of the effect which becomes vanishing once the Lifschitz transition occurs. As expected the appearance of the APHE occurs when the Fermi energy crosses the point in which the two energy bands are the closest, coinciding with the largest source of Berry curvature in momentum space.

As discussed above, in the mirror symmetric case the Berry curvature is odd with respect to the mirror line forcing its integral to be vanishing. The Berry curvature dipole, on the other hand, is expected to be non-zero. Since the mirror symmetry implies the presence of a band touching point, the dipole density, $\partial_{k_{\alpha}} \Omega_{ \pm}^{z}(\mathbf{k})$, is divergent in the neighbourhood of the degeneracy boosting the value of the dipole, and hence the non-linear response. If, for example, the magnetic field is placed along $\hat{x}(\phi=0)$, the dipole density diverges as $\partial_{k_{x}} \Omega_{ \pm}^{z}(\mathbf{k}) \sim \pm \operatorname{sign}\left(k_{y}\right) / k_{y}^{2}$ where $k_{y}$ is centered around the degeneracy point along the mirror line. This divergence, of course, is never really achieved in a real material where scattering effects should attenuate 


\subsection{Anomalous Planar Hall Effect}

(a)

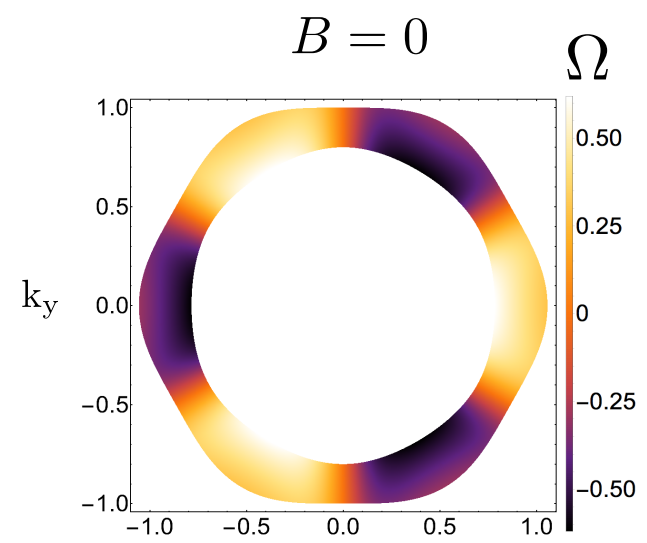

(b)

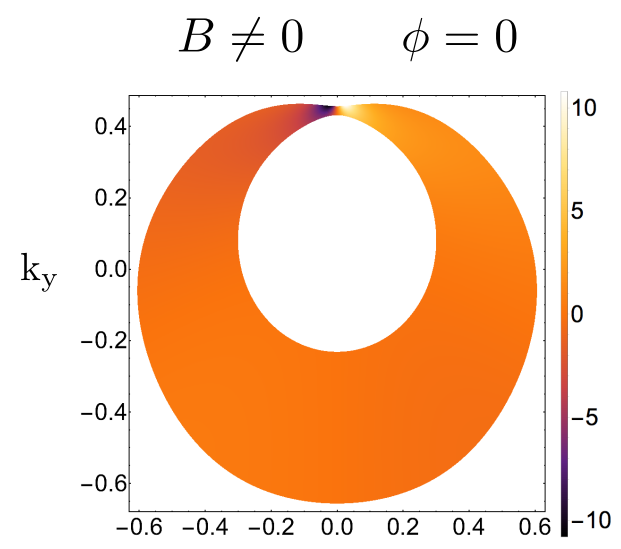

(c)

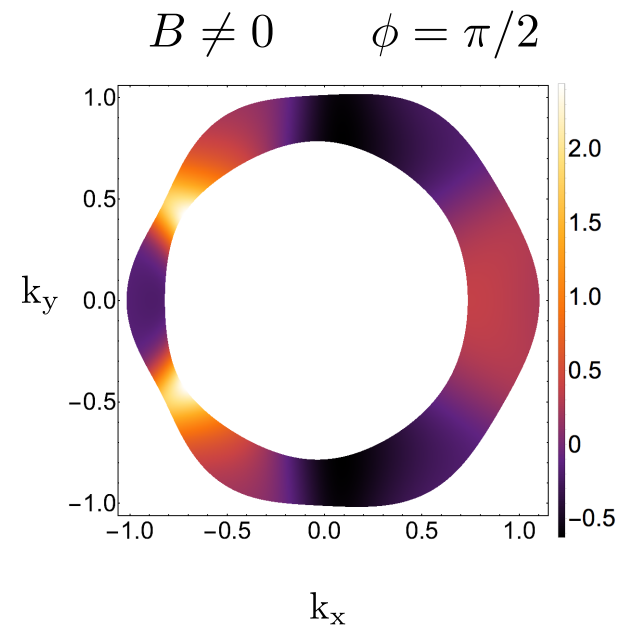

Figure 4.3: Total Berry curvature of the of the warped Rashba Hamiltonian with Zeeman coupling at fixed Fermi energy. 
this behaviour.

(a)

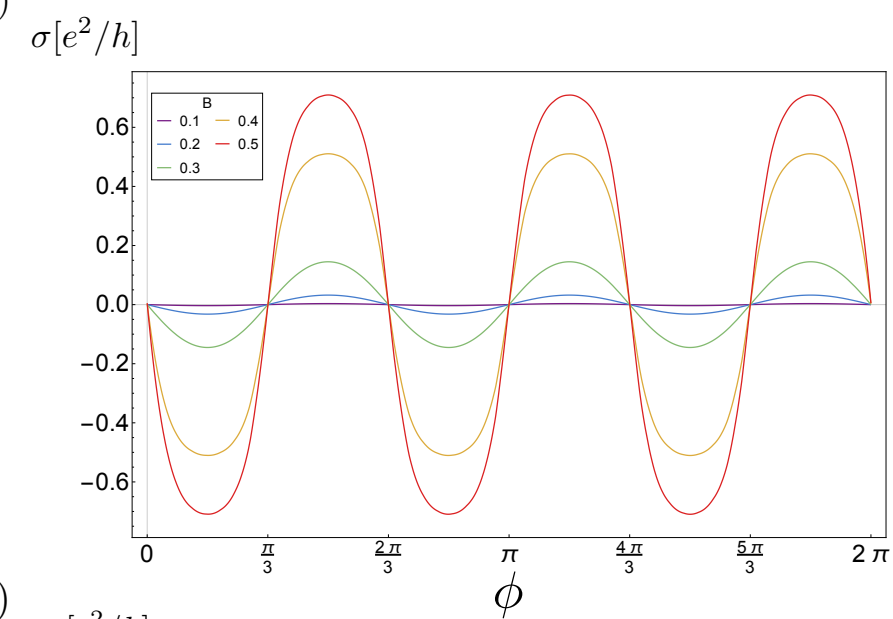

(b)

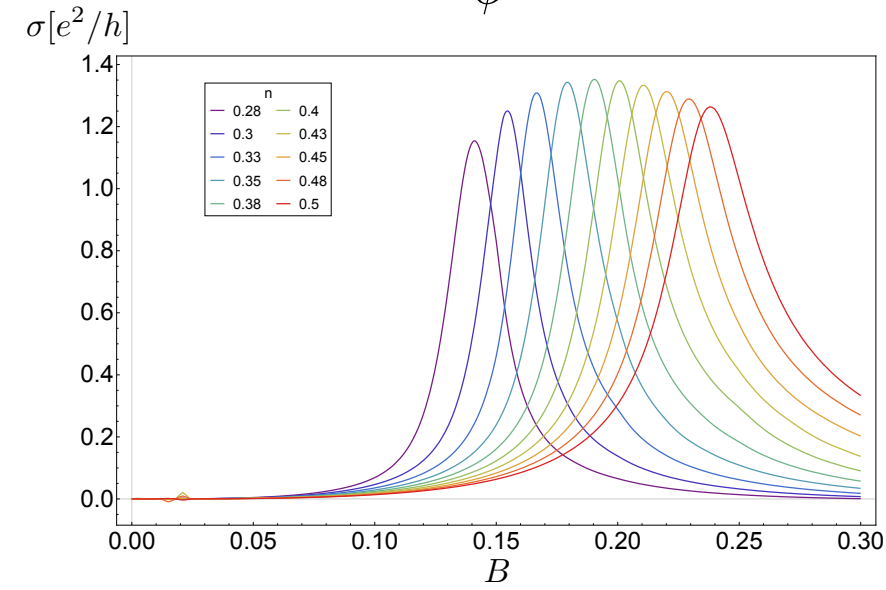

Figure 4.4: (a)Angular dependence of the $\sigma_{\mathrm{APHE}}$ for different values of the magnetic field $B$. (b) Magnetic field dependence of the $\sigma_{\mathrm{APHE}}$ at fixed carrier density $n$ for $\phi=\pi / 2$. 


\section{Spin Field-Effect Transistor in a Quantum Spin-Hall Device}

Spintronics [91] is the field dedicated to studying how to actively control and manipulate the electronic spin degree of freedom in solid-state systems. When spin-orbit coupling (SOC) is present, the electron's spin and momentum are locked to each other allowing to study the interplay between charge and spin degrees of freedom. This interplay is of central interest since it opens the possibility to manipulate electric currents by controlling the electronic spin and viceversa, paving the way to the development of new devices of technological relevance. The first proposal of a spintronic device with electrical spin manipulation was the spin field-effect transistor, brought forward by Datta and Das [92]. Since then, spin transistors have been the subject of intense research and, to date, are still a central problem in the field of spintronics. In recent years, much attention has been drawn to exploit spin interference effects on the electronic transport properties of mesoscopic devices with loop geometries, such as semiconducting quantum rings $[7,8,93-97]$. In the presence of electromagnetic potentials, the conductance of a semiconductor ring exhibits signs of quantum interference due to the Aharonov-Bohm [98] (A-B) and Aharonov-Casher [99](A-C) effects. Being manifestations of the Berry phase, these interference effects have been exploited to detect in the laboratory the spin geometric phase $[8,100]$.

An alternative setup for the detection of the $\pi$-Berry phase was proposed in Ref. [101], with the interferometer based on a quantum spin-Hall (QSH) insulator. The QSH insulator [21] is a time-reversal symmetric topological state of matter which possesses in gap helical edge states: at each edge of the system there are two counter-propagating states with opposite spin projections. The realization of QSH insulators in HgTe [22] and InAs/GaSb/AlSb [102] quantum wells has since opened the possibility to engineer new types of spin transistors. A notable example has been discussed in Ref. [103] where by combining the helical nature of the QSH edge states with the A-B effect, a setup that behaves as a spin transistor has been proposed. One of the 
main advantages of using a QSH insulator lies in the fact that transport in the system is ballistic in nature and takes place along the edges, making it effectively a one-dimensional (1D) system. In this work we consider an alternative QSH-based setup exploiting the effect of the Rashba spin-orbit coupling (SOC), originating from structural inversion asymmetry. The presence of Rashba SOC [104] breaks the axial spin symmetry of the helical edge states, tilting their spin projection in the QSH plane. Consequently, in a disk geometry the Rashba SOC yields a local rotation of the spin projection of the helical edge states along the disk. By further controlling the Rashba strength through an additional external gate voltage it is possible to both modulate electric currents which pass through the QSH insulator and manipulate the spin projection of single incoming electrons, similarly to the A-B based setup of Ref. [103]. By studying the transport properties of the QSH insulator in the presence of Rashba SOC we will indeed show how such a system can be used as an all-electric spin transistor. It is interesting to note that since there are only two counter-propagating modes at the edge, the QSH insulator can be seen as a faithful implementation of the original Datta-Das transistor: the original device introduced in Ref. [92] drew inspiration from an electro-optic modulator in which polarized light was split into two beams that suffered different phase shifts. In this sense the QSH is a true electronic analog of the electro-optic modulator.

The chapter is organized as follows: in Section 5.1 we study the BernevigHughes-Zhang [21] (BHZ) Hamiltonian with the addition of a linear Rashba SOC term. We find the in gap eigenstates and eigenvalues of the system in a disk geometry and show how the Rashba coupling is responsible for the tilting of the spin projection of the two helical edge states. In Section 5.2 we study an effective 1D model of the QSH disk and calculate the conductance of the system through the Landauer approach. We show that the QSH disk behaves as a spin field-effect transistor. Finally in Section 5.3, by using the microscopic tight-binding BHZ model, we validate our findings through a numerical calculation of the conductance.

\subsection{In Gap States of the BHZ Hamiltonian}

We begin by studying the properties of the helical edge states of a QSH insulator disk in the presence of Rashba SOC [105-107]. It is well known 
[22] that the QSH phase occurs in the "inverted" regime of $\mathrm{HgTe} / \mathrm{CdTe}$ semiconductor quantum wells, which is achieved by tuning the thickness of the HgTe well above a critical thickness $d_{c} \simeq 6 \mathrm{~nm}$. The occurrence of this topological phase transition can be captured using conventional $\mathbf{k} \cdot \mathbf{p}$ theory. Starting from the 6-band Kane model [108] and using perturbation theory near the $\Gamma$ point one can obtain an effective four band model $\left(\left|E 1, j_{z}= \pm \frac{1}{2}\right\rangle\right.$, $\left.\left|H 1, j_{z}= \pm \frac{3}{2}\right\rangle\right)$ for the subbands of the quantum well structure [21]. The $\left.E 1, j_{z}= \pm \frac{1}{2}\right\rangle$ subbands are a linear combination of s-like $\left|\Gamma_{6}, j_{z}= \pm \frac{1}{2}\right\rangle$ and the light hole $\left|\Gamma_{8}, j_{z}= \pm \frac{1}{2}\right\rangle$ bands while the $\left|H 1, j_{z}= \pm \frac{3}{2}\right\rangle$ come about from the $\left|\Gamma_{8}, j_{z}= \pm \frac{3}{2}\right\rangle$ heavy hole bands. The electronic structure is then described by the effective BHZ Hamiltonian,

$$
\mathcal{H}_{B H Z}=\left(\begin{array}{cccc}
\epsilon_{\mathbf{k}}+M_{\mathbf{k}} & A k_{+} & 0 & 0 \\
A k_{-} & \epsilon_{\mathbf{k}}-M_{\mathbf{k}} & 0 & 0 \\
0 & 0 & \epsilon_{\mathbf{k}}+M_{\mathbf{k}} & -A k_{-} \\
0 & 0 & -A k_{+} & \epsilon_{\mathbf{k}}-M_{\mathbf{k}}
\end{array}\right) .
$$

Eq. 5.1 is written in the basis $\left|E 1, j_{z}=+\frac{1}{2}\right\rangle,\left|H 1, j_{z}=+\frac{3}{2}\right\rangle,\left|E 1, j_{z}=-\frac{1}{2}\right\rangle$ and $\left|H 1, j_{z}=-\frac{3}{2}\right\rangle$. We have defined $\epsilon_{\mathbf{k}}=C-D\left(k_{x}^{2}+k_{y}^{2}\right), M_{\mathbf{k}}=M-$ $B\left(k_{x}^{2}+k_{y}^{2}\right)$ and $A, B, C, D$ and $M$ are model parameters. We have also introduced $k_{ \pm}=k_{x} \pm i k_{y}$ with $k_{x, y}=-i \partial_{x, y}$. For simplicity, we set $C=$ $D=0$. The Hamiltonian above preserves time-reversal symmetry, with the time reversal symmetry operator defined as $\Theta=-i\left(\sigma_{y} \otimes \sigma_{0}\right) \mathcal{K}$, where $\mathcal{K}$ stands for complex conjugation and $\sigma_{\alpha}$ are the Pauli matrices. When $\operatorname{sign}(B)=\operatorname{sign}(M)$ the system is in the QSH phase and is characterized by a non-trivial $\mathbb{Z}_{2}$ topological invariant. The presence of structural inversion asymmetry gives rise to Rashba terms [104] which couple the two spin blocks in Eq. 5.1 and break the axial spin symmetry. At linear order in $\mathbf{k}$ the Rashba Hamiltonian only couples the $\left|E 1, j_{z}= \pm \frac{1}{2}\right\rangle$ bands,

$$
\mathcal{H}_{R}=\left(\begin{array}{cccc}
0 & 0 & -i \alpha_{R} k_{-} & 0 \\
0 & 0 & 0 & 0 \\
i \alpha_{R} k_{+} & 0 & 0 & 0 \\
0 & 0 & 0 & 0
\end{array}\right) .
$$

It easy to see that unlike $\mathcal{H}_{B H Z}, \mathcal{H}_{R}$ breaks the effective two-dimensional inversion symmetry, $\mathcal{H}_{R}(k) \neq \mathcal{I}_{2 D} \mathcal{H}_{R}(-k) \mathcal{I}_{2 D}$, where $\mathcal{I}_{2 D}=\sigma_{z} \otimes \sigma_{0}$ is the inversion operator.

To find the helical edge states dispersion of the full Hamiltonian $\mathcal{H}=$ 
5 Spin Field-Effect Transistor in a Quantum Spin-Hall Device

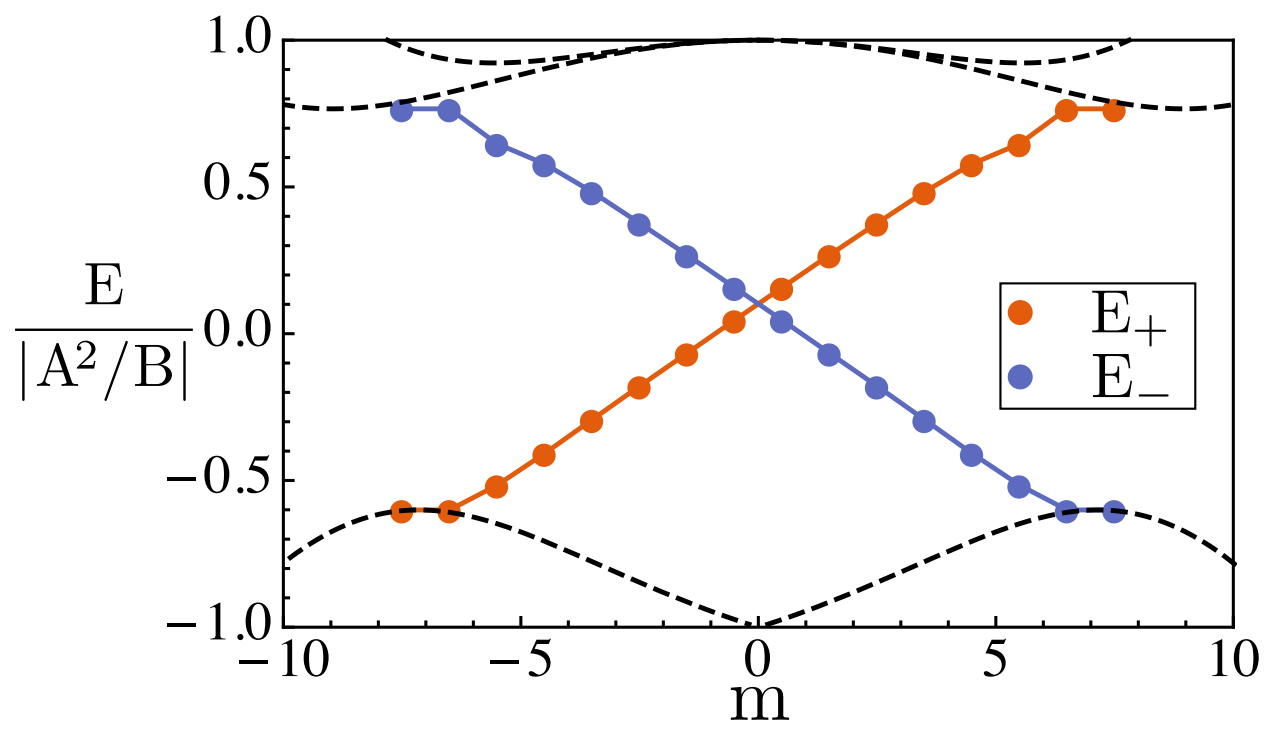

Figure 5.1: Energy dispersion of the two in-gap helical edge states of a QSH insulator in a disk geometry as a function of the half-integer eigenvalue $\mathrm{m}$ of the operator $\Sigma_{z} . E_{ \pm}$indicate respectively clockwise movers and counter-clockwise movers. The dashed lines represent the bulk bands. The value of the Rashba strength is set to $\alpha_{R}=|A| / 2$. The maximum value of the Rashba strength compatible with the presence of a full bulk band gap is $\alpha_{R}^{\max }=2|A|$. Energies have been measured in units of $\left|A^{2} / B\right|$. 
$\mathcal{H}_{B H Z}+\mathcal{H}_{R}$ in a disk geometry, we write $\mathcal{H}$ in polar coordinates $(r, \phi)$ :

$$
\mathcal{H}=\left(\begin{array}{cccc}
B \Pi+M & -i A \Lambda^{+} & -\alpha_{R} \Lambda^{-} & 0 \\
-i A \Lambda^{-} & -B \Pi-M & 0 & 0 \\
\alpha_{R} \Lambda^{+} & 0 & B \Pi+M & i A \Lambda^{-} \\
0 & 0 & i A \Lambda^{+} & -B \Pi-M
\end{array}\right)
$$

where

$$
\begin{aligned}
& \Pi=\left(\partial_{r}^{2}+\frac{1}{r} \partial_{r}+\frac{1}{r^{2}} \partial_{\phi}^{2}\right), \\
& \Lambda^{+}=e^{i \phi}\left(\partial_{r}+\frac{i}{r} \partial_{\phi}\right), \\
& \Lambda^{-}=e^{-i \phi}\left(\partial_{r}-\frac{i}{r} \partial_{\phi}\right) .
\end{aligned}
$$

We are now interested in solving the Schrödinger equation $\mathcal{H} \psi(r, \phi)=E \psi(r, \phi)$ for energies inside the insulating bulk gap. In order to diagonalize the Hamiltonian of Eq. 5.3 we first show that the problem is separable in the two variables $r$ and $\phi$. The total electronic angular momentum is given by the composition of the spin angular momentum with the orbital angular momentum, $\mathbf{J}=\mathbf{L}+\mathbf{S}$, plus the angular momentum $\mathbf{L}_{\phi}$ due to the rotation around the disk. Its projection along the $\hat{z}$ axis is given by the operator $\Sigma_{z}=L_{\phi}+J_{z}$, with $L_{\phi}=-i \partial_{\phi}$ and $J_{z}=\operatorname{diag}\left[\frac{1}{2}, \frac{3}{2},-\frac{1}{2},-\frac{3}{2}\right]$. It is straightforward to show that $\left[\mathcal{H}, \Sigma_{z}\right]=0$, and hence our wave functions can be written as eigenvectors of $\Sigma_{z}$. The in gap solutions for Eq. 5.3 take the form,

$$
\psi_{m}^{\xi}(r, \phi)=\left(\begin{array}{l}
e^{i\left(m-\frac{1}{2}\right) \phi} c_{1}(\xi) I_{m-\frac{1}{2}}(\xi r) \\
e^{i\left(m-\frac{3}{2}\right) \phi} c_{2}(\xi) I_{m-\frac{3}{2}}(\xi r) \\
e^{i\left(m+\frac{1}{2}\right) \phi} c_{3}(\xi) I_{m+\frac{1}{2}}(\xi r) \\
e^{i\left(m+\frac{3}{2}\right) \phi} c_{4}(\xi) I_{m+\frac{3}{2}}(\xi r)
\end{array}\right)
$$

where $m$ is the half-integer eigenvalue of the operator $\Sigma_{z}$ ensuring the $2 \pi$ periodicity of the wavefunctions, whereas $I_{m}(r)$ are the modified Bessel functions of the first kind, necessary to have a normalizable solution. Finally, $c$ and $\xi$ are constants which depend on the system's parameters and energy. For a given in gap energy $E$ we find four values of $\xi$ for which the wavefunctions $\psi_{m}^{\xi}$ are linearly independent: the total wave function can then be 
5 Spin Field-Effect Transistor in a Quantum Spin-Hall Device

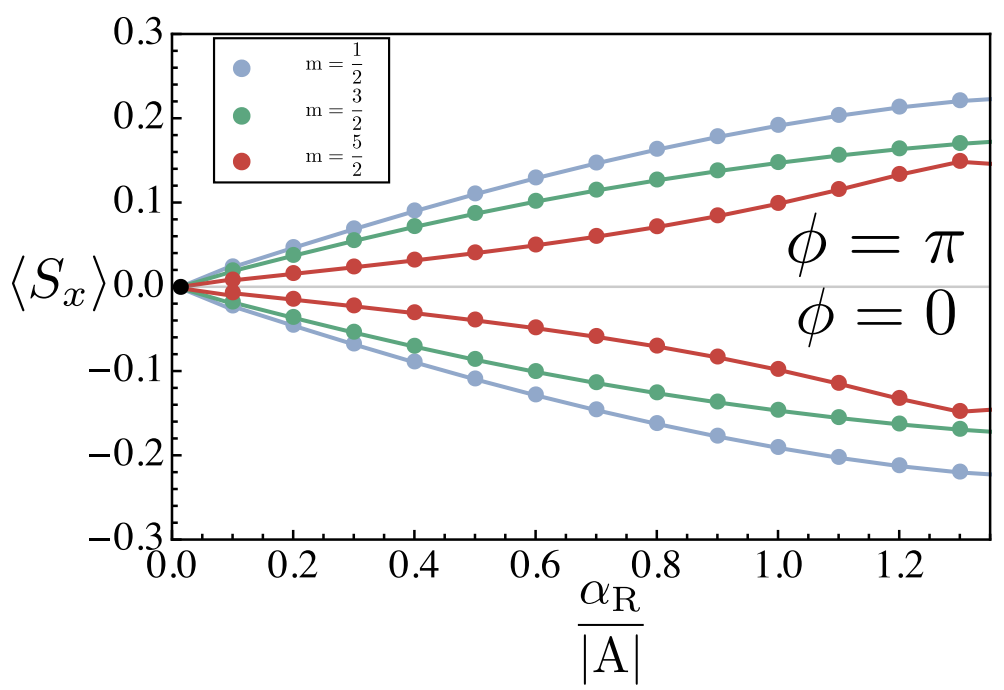

Figure 5.2: Local expectation value of $S_{x}$ for different counter-clockwise moving states as a function of the Rashba strength $\alpha_{R}$. The spin projection is measured at two opposite points of the disk, $\phi=0$ and $\phi=\pi$, where the only non-zero in-plane spin component is given by $S_{x}$. The values of $\left\langle S_{x}\right\rangle$ have been computed numerically and are represented by dots in the above graph; lines joining the dots are present only as a guide for the eye.

written as a linear combination,

$$
\Phi_{m}(r, \phi)=\sum_{i=1}^{4} a_{i} \psi_{m}^{\xi_{i}}(r, \phi) .
$$

To find the in gap eigenvalues we impose fixed boundary conditions at the edge of the disk: $\Phi_{m}\left(r=r_{0}, \phi\right)=0$. Fig. 5.1 shows the energy dispersion of the helical edge states in the QSH phase. Away from the energy bulk the dispersion is practically linear as conventionally found in ribbon geometries $[106,109]$. The presence of the Rashba SOC breaks the axial spin symmetry of the BHZ Hamiltonian and tilts the electronic spin towards the QSH plane. This can be seen by computing the out-of-plane spin component $S_{z}=\frac{1}{2} \sigma_{0} \otimes$ $\sigma_{z}$ : the local expectation value $\left\langle S_{z}\right\rangle$ decrease monotonically by increasing 
the Rashba strength. Moreover, in the current disk geometry, the in-plane spin component is reversed under a $\pi$ rotation. For instance, at $\phi=0$ and $\phi=\pi$ the only in-plane component corresponds to $S_{x}=\frac{1}{2} \sigma_{0} \otimes \sigma_{x}$ and as shown in Fig. 5.2 it is completely reversed after half a turn.

We are now interested in using this spin-tilt effect to study the electronic transport through the disk when the Fermi energy is in the bulk band gap. In this case the QSH behaves effectively as a 1D single mode ballistic conductor with only two counter-propagating states at the edge. For this reason it is inherently different from a quasi-1D semiconductor ring [7]. In the latter, for all Fermi energies, there are two clockwise movers and two counter-clockwise movers (neglecting transverse modes). As we will show, the difference in number of propagating channels between the two systems will result in distinct transport properties.

A qualitative understanding of electronic transport through the QSH can be achieved by considering an effective 1D model that retains the helical nature of the edge states and the spin-tilting mechanism of the Rashba SOC.

\subsection{Effective 1D Model}

\subsubsection{D Hamiltonian}

In order to have two counter-propagating helical modes which mimic the QSH edge states and a Rashba spin-tilting mechanism we study the following effective 1D Hamiltonian,

$$
\mathcal{H}_{e f f}=-\frac{i \hbar}{2}\left\{\omega_{z} \sigma_{z}+\omega_{R} \sigma_{r}, \partial_{\phi}\right\}
$$

where $\{$,$\} is the anticommutator, necessary to have an hermitian Hamilto-$ nian $[11,110]$. In Eq. 5.6 we have defined the two characteristic frequencies $\omega_{z}=\frac{v_{F}}{r_{0}}$ (with $v_{F}$ Fermi velocity of the edge states and $r_{0}$ disk radius) and $\omega_{R}=\frac{2 \alpha_{R}}{\hbar r_{0}}$. The radial Pauli matrix is defined as $\sigma_{r}=\cos \phi \sigma_{x}+\sin \phi \sigma_{y}$. The eigenvalues of Eq. 5.6 are,

$$
E_{ \pm}(m)=\hbar \omega_{z}\left[-\frac{1}{2} \pm m \sqrt{1+\left(\frac{\omega_{R}}{\omega_{z}}\right)^{2}}\right]
$$


The spectrum, much like the one in Fig. 5.1, is linear in $m$ and obeys timereversal symmetry: at each energy $E$ there are two corresponding eigenstates with opposite spin projections and opposite velocities. The eigenstates of Eq. 5.6 are,

$$
\begin{array}{r}
\psi_{m}^{+}(\phi)=e^{i m \phi}\left(\begin{array}{c}
e^{-i \frac{\phi}{2}} \cos \frac{\gamma}{2} \\
e^{i \frac{\phi}{2}} \sin \frac{\gamma}{2}
\end{array}\right) \\
\psi_{m}^{-}(\phi)=e^{i m \phi}\left(\begin{array}{c}
-e^{-i \frac{\phi}{2}} \sin \frac{\gamma}{2} \\
e^{i \frac{\phi}{2}} \cos \frac{\gamma}{2}
\end{array}\right)
\end{array}
$$

where $m$ is the half-integer eigenvalue of the operator $\Sigma_{z}^{e f f}=-i \partial_{\phi}+$ $\frac{\sigma_{z}}{2}$. The angle $\gamma=\arctan \left(\frac{\omega_{R}}{\omega_{z}}\right)$ measures the spin tilt with respect to the quantization axis $\hat{z}$ : at zero Rashba $(\gamma=0)$ the spinors in Eqs. 5.8 and 5.9 simply reduce to $|\uparrow, \downarrow\rangle$ eigenstates of $\sigma_{z}$. This is in agreement with the zero Rashba behaviour of the BHZ Hamiltonian of Eq. 5.1, where the edge states are eigenstates of $S_{z}=\frac{1}{2} \sigma_{0} \otimes \sigma_{z}$. In the following we will take into account eigenstates which lie far from the bulk bands, close to zero energy, where the dispersion is mostly linear. For these states the spin tilting effect is larger, as shown in Fig. 5.2. Under these assumptions we can safely describe the BHZ helical edge states with the effective 1D Hamiltonian of Eq. 5.6.

\subsubsection{Scattering Matrix Approach}

The transport properties of the system at zero temperature are studied by coupling symmetrically the QSH disk to two semi-infinite ballistic leads. By applying a low bias we calculate the unpolarized conductance using the Landauer formula [13],

$$
G=\frac{e^{2}}{h} \sum_{\sigma, \sigma^{\prime}} T_{\sigma \sigma^{\prime}}
$$

where $e$ is the electron charge, $h$ is the Planck constant and $T_{\sigma \sigma^{\prime}}$ denotes the transmission probability between incoming $\sigma$ and outgoing $\sigma^{\prime}$ states in the leads. Following closely the setup of Ref. [103], we consider ferromagnetic leads with in-plane magnetization in order to inject polarized spins in the left lead and detect spins polarized along the polar angle $\hat{\theta}$ in the right lead. A schematic picture of the setup is shown in Fig. 5.3. The Hamiltonian of the 

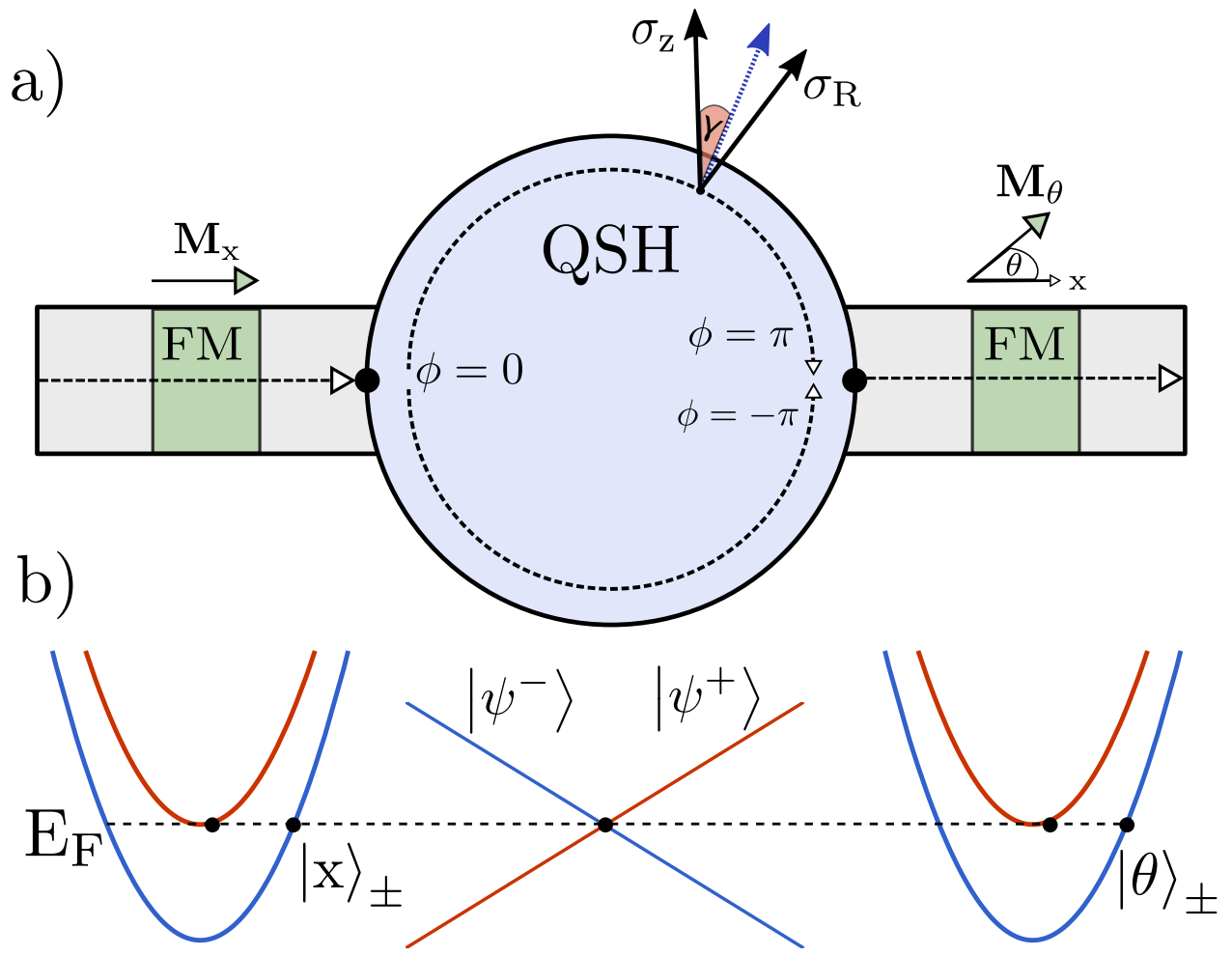

Figure 5.3: a) QSH spin field effect transistor setup. The two semi-infinite ferromagnetic leads are coupled symmetrically at $\phi=0$ and $\phi=\pi$. Magnetizations in the left and right leads are given by the direction of $M$. Inside the disk, the electron spin is tilted by an angle $\gamma$ in the direction of the Rashba field. (b) Schematic energy dispersion of $\mathcal{H}_{F M}$ and $\mathcal{H}_{\text {eff }}$. 


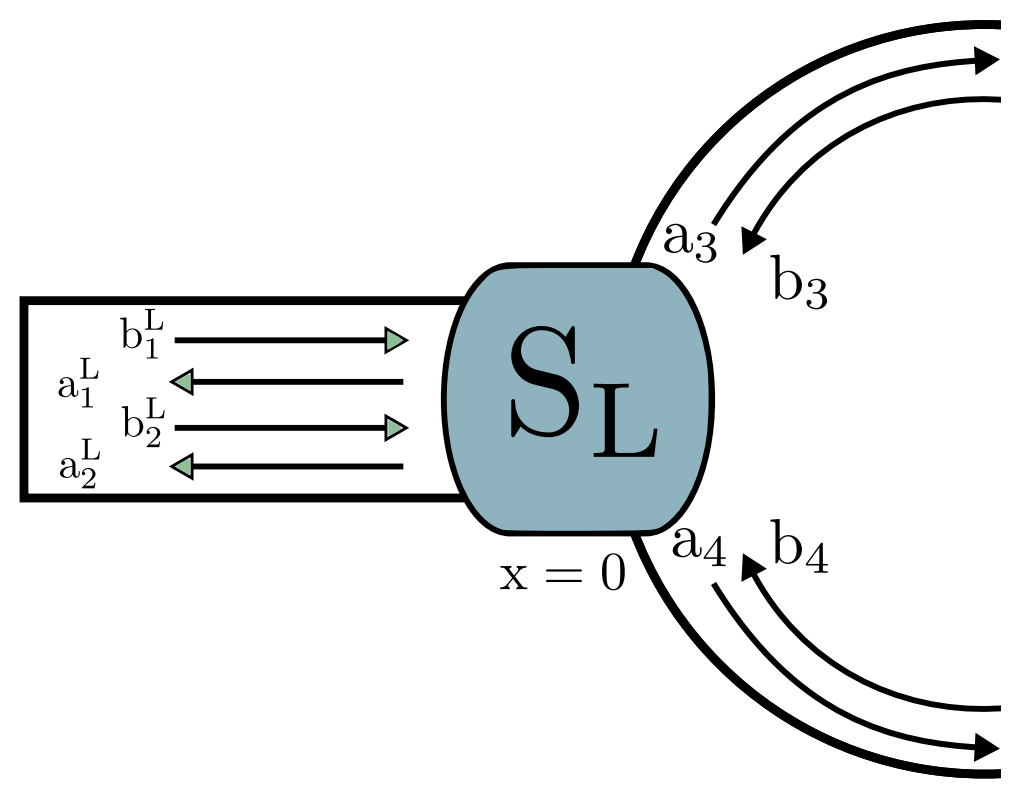

Figure 5.4: Schematic representation of the incoming and outgoing modes in the left junction of the system.

ferromagnetic leads, $\mathcal{H}_{F M}=\frac{p^{2}}{2 m} \sigma_{0}+\mathbf{M}(\theta) \cdot \boldsymbol{\sigma}$, contains a Zeeman-splitting term proportional to the magnetization vector $\mathbf{M}(\theta)=M(\cos \theta, \sin \theta, 0)$ and a vector of Pauli matrices $\sigma$. Eigenstates in the left lead have the form $|x\rangle_{ \pm}=\frac{e^{i k x}}{\sqrt{2}}(1, \pm 1)^{T}$, while in the right lead $|\theta\rangle_{ \pm}=\frac{e^{i k x}}{\sqrt{2}}\left(e^{-i \theta}, \pm 1\right)^{T}$. The QSH region is described by Eq. 5.6, where in the open geometry setup the quantum number $m$ labelling the eigenstates in Eqs. 5.8 and 5.9 will no longer be quantized. The scattering matrix of the system can be obtained from the knowledge of the scattering matrices at the QSH-injector interface $S_{L}$, and at the QSH-detector interface $S_{R}$. To calculate them we first notice that if both the spin majority and spin minority bands are occupied there are two right-moving states and two left-moving states in the ferromagnetic leads. The same number of propagating states are found in the QSH disk: each arm of the disk has one clockwise mover and one counter-clockwise mover with opposite spin projections. (Fig. 5.4). Hence $S_{L}$ and $S_{R}$ are $4 \times 4$ matrices whose elements can be calculated imposing current conservation for 
each scattering state at the interface. This condition leads to 16 equations for the 16 elements of $S_{L, R}$. For example the scattering ansatz for a rightmoving state in the left junction can then be written as,

$$
\left\{\begin{array}{l}
\phi_{F M, \mathcal{R}}^{\lambda}(x)=\frac{\chi_{F M, \mathcal{R}}^{\lambda}}{\sqrt{\left|v_{F M}^{\lambda}\right|}} e^{i k_{\lambda} x}+\sum_{\lambda^{\prime}} r_{\lambda, \lambda^{\prime}} \frac{\chi_{F M, \mathcal{L}}^{\lambda^{\prime}}}{\sqrt{\left|v_{F M}^{\lambda^{\prime}}\right|}} e^{-i k_{\lambda^{\prime}} x} \\
\phi_{Q S H, \mathcal{R}}^{\lambda}(x)=\sum_{\lambda^{\prime}} t_{\lambda, \lambda^{\prime}} \frac{\chi_{Q S H, \mathcal{R}}^{\lambda^{\prime}}}{\sqrt{\left|v_{Q S H}^{\lambda^{\prime}}\right|}} e^{i k_{\lambda^{\prime}} x}
\end{array}\right.
$$

where $\lambda= \pm$ labels the two possible modes, which in general will have different spinorial parts $\chi^{\lambda}$ (see Eqs. 5.8 and 5.9) and velocities $v^{\lambda}$. The indices $\mathcal{R}, \mathcal{L}$ discriminate between right-moving and left-moving states. Since the lead and the disk are parametrized by two different coordinate systems we choose to label the eigenstates with their wave number $k$, which can be simply written as the ratio between the angular momentum $m$ and the disk radius $r_{0}$. The coefficients $r_{\lambda, \lambda^{\prime}}$ and $t_{\lambda, \lambda^{\prime}}$ are the probability amplitudes that a state $\lambda$ will be reflected or transmitted in a state $\lambda^{\prime}$. Each propagating state is normalized to unit flux in order to obtain a unitary S-matrix. A similar ansatz holds for the right junction.

Once both S-matrices are calculated, they can be combined to obtain the full scattering matrix of the device,

$$
\left(\begin{array}{c}
b_{i}^{L} \\
b_{i}^{R}
\end{array}\right)=\left(\begin{array}{cc}
r & t^{\prime} \\
t & r^{\prime}
\end{array}\right)\left(\begin{array}{c}
a_{i}^{L} \\
a_{i}^{R}
\end{array}\right)
$$

where $b_{i}^{L, R}$ and $a_{i}^{L, R}(i=1,2)$ are respectively the wave amplitudes of the two outgoing and ingoing states in the left $L$ and right $R$ lead. We define $r$ and $t$ as the $2 \times 2$ matrices whose elements are the spin-dependent reflection and transmission amplitudes. The transmission coefficients in Eq. 5.10 are just the modulus square of the elements of $t$. The unpolarized conductance from left to right lead can be then simply expressed as [13] $G=\frac{e^{2}}{h} \operatorname{tr}\left(t t^{\dagger}\right)$.

At zero Rashba, $\gamma=0$, and with both ferromagnets aligned we find perfect transmission, $T=\operatorname{diag}[1,1]$, when the momenta of the states in the QSH disk satisfy the condition $k r_{0}=\frac{\mathbb{Z}}{2}$, with $\mathbb{Z}$ an integer. This resonance effect can be understood by noticing that for $m=\frac{\mathbb{Z}}{2}$ the state inside the QSH disk is also an eigenstate of the closed system, as shown in Section 5.2.1. In the remainder, we will fix the Fermi energy of the system to fulfil the 
resonance condition in the disk. We emphasize that for a sufficiently large disk radius the in gap eigenstates of Eq. 5.7 are close enough that resonance is achieved for almost any value of the Fermi energy. Calculating $G$ under this assumption yields the density plot in Fig. 5.5, where the unpolarized conductance is modulated as a function of the Rashba strength $\gamma$ and the relative magnetization $\theta$ between the two leads. Here the Fermi energy has been fixed close to the bottom of the upper band in the leads, in order to have a large modulation of the unpolarized conductance. If we were to raise the Fermi energy in the leads such that the wave numbers of the two spin-polarized injected electrons were comparable, then the modulation of the conductance would be largely suppressed, and the ballistic conductance, independent of the Rashba strength and the relative magnetization between injector and detector, would be quantized to $2 e^{2} / h$.

The density plot of the unpolarized conductance shows that when the ferromagnetic leads have opposite magnetizations $(\theta=\pi)$, the maximum conductance is reached as the Rashba field is strong enough to completely flip the spin of the incoming electrons, that is for $\gamma \rightarrow \frac{\pi}{2}$. Hence for sufficiently large Rashba couplings the electron spin is reversed: this effect is due to the phase accumulated after half a turn by the two eigenstates in the QSH. From the elements of the $t$ matrix we can also compute the contribution to the conductance of an injected spin polarized current. This polarized conductance turns out to be half the value of the unpolarized one, regardless of the sign of the spin of the injected carrier. This suggests that at the resonance condition the conductance modulation is dominated by the spin texture of the helical edge states inside the QSH: at resonance the injected electrons always enter the QSH, but they can only transfer to the right lead if their spin projections can match the ones of the detector. By making use of these observations we can now calculate analytically the spin-polarized conductance.

\subsubsection{Spin-Polarized Conductance}

Having established that at resonance carriers with opposite spin polarization contribute equally to the conductance, we can restrict ourselves to investigate the modulation of the spin polarized conductance, and assume that only the lowest Zeeman band is occupied. Since at resonance injected electrons enter the QSH unimpeded, transmission is then determined by the spin 


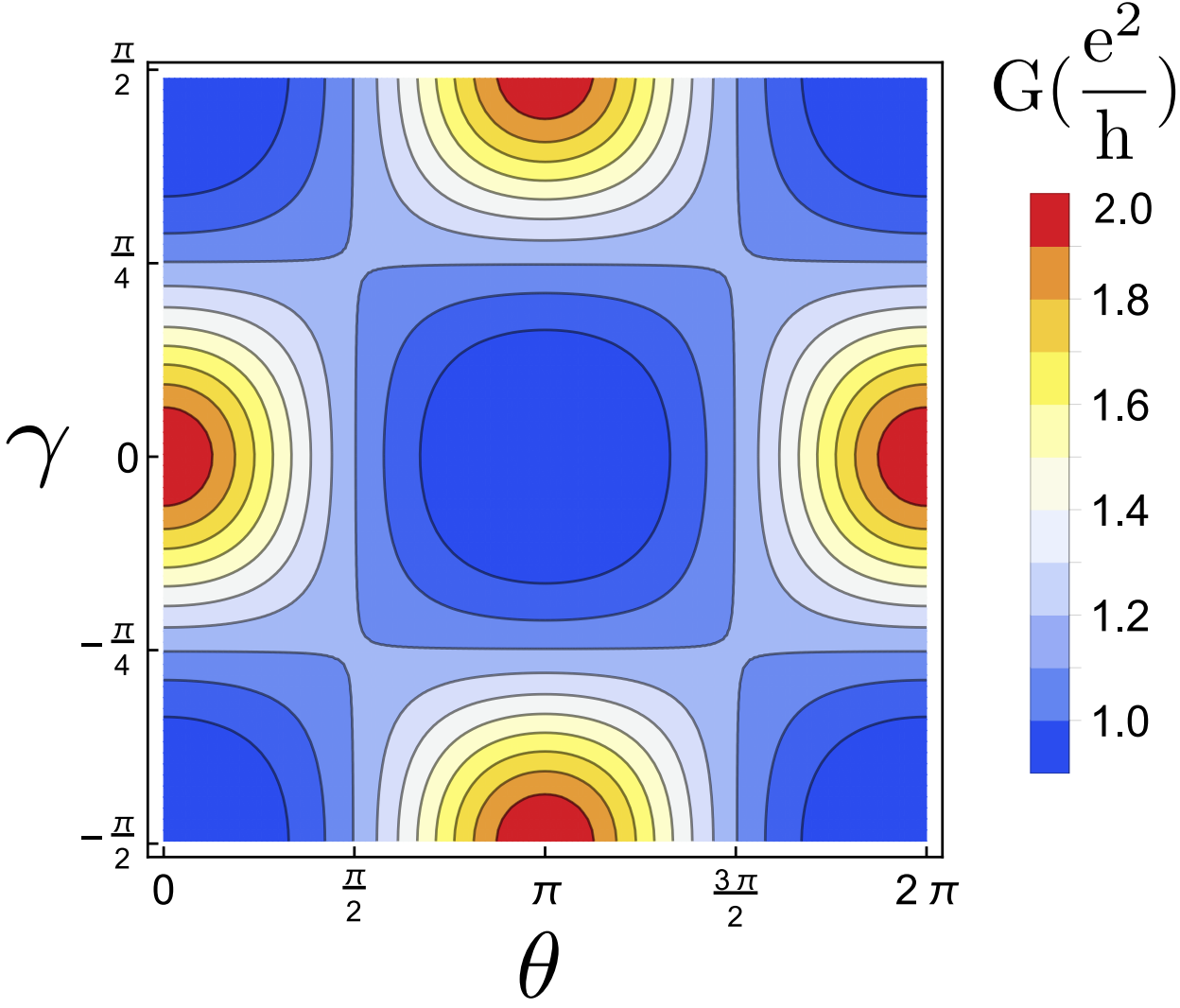

Figure 5.5: Density plot of the unpolarized conductance as a function of the spin tilt $\gamma$ and the magnetization angle $\theta$ of the right ferromagnetic lead. 
projection of electrons upon exiting the disk at $\phi=\pi$. Hence, the conductance modulation is controlled solely by the spin structure of the helical edge states, greatly simplifying the description of the transmission coefficient and leading to a clear analytic understanding of the physics of the system.

To calculate the transmission coefficient $T_{\sigma \sigma^{\prime}}$ we follow the steps of Ref. [7]. The spin eigenstates $|x\rangle$ incoming from the left lead propagate coherently in the disk, through the helical edge states, and leave the disk in a mixed spin state $\left|\sigma_{\text {out }}\right\rangle=\sum_{\lambda= \pm}\left\langle\psi_{m}^{\lambda}(0)|| x\right\rangle\left|\psi_{m}^{\lambda}(\lambda \pi)\right\rangle$. The transmission coefficient can then be obtained from the overlap between $\left|\sigma_{\text {out }}\right\rangle$ and the outgoing eigenstate $|\theta\rangle$ in the right lead, $T_{x \theta}=\left|\left\langle\theta \mid \sigma_{\text {out }}\right\rangle\right|^{2}$. The polarized conductance takes the form,

$$
G_{x \theta}(\gamma, \theta)=\frac{e^{2}}{2 h}(1+\cos 2 \gamma \cos \theta) .
$$

The above conductance has the same modulation pattern of the one found in Fig 5.5. As expected at strong Rashba SOC, $\gamma \rightarrow \frac{\pi}{2}$, the incoming electron spin is completely reversed.

To calculate the unpolarized conductance one must sum over all possible spin polarizations in the left and right leads. The calculation yields a conductance $G=\frac{2 e^{2}}{h}$, which is simply the inverse contact resistance of a single mode conductor. Since the wave numbers of the injected and detected electrons do not play a role in this calculation, this result follows only from the helical nature of the two propagating edge modes: the two modes are orthogonal and cannot interfere with each other. Contrary to a conventional 1D semiconductor ring, an interference pattern is therefore absent. This is due to the fact that in each arm of the semiconductor ring there are double the movers than the ones in the QSH. When the two arms of the ring recombine at $\phi=\pi$, electrons coming from different arms, with same spin projection, can interfere leading to a modulation of $G$. We point out that, as mentioned earlier, this result is in agreement with the scattering matrix approach analysis assuming the Fermi energy in the leads is such that the wave numbers of the incoming electrons are comparable. We now validate numerically our results by studying a microscopic tight-binding model corresponding to a regularized version of Eq. 5.1. 


\subsection{D Tight-Binding Model}

To corroborate our findings we perform a numerical calculation of the transport properties of our device. The QSH disk is described using the BHZ tight-binding model on a square lattice,

$$
\begin{aligned}
\mathcal{H}^{t b}= & \sum_{i, j} c_{i, j}^{\dagger} c_{i, j} \hat{V}+\sum_{i, j} c_{i+1, j}^{\dagger} c_{i, j} \hat{T}_{x} \\
& +\sum_{i, j} c_{i, j+1}^{\dagger} c_{i, j} \hat{T}_{y}+h . c .
\end{aligned}
$$

where,

$$
\begin{aligned}
& \hat{V}=\mu \mathcal{I}_{4 \times 4}+\left(M-\frac{4 B}{a^{2}}\right) \sigma_{z} \otimes \sigma_{0}, \\
& \hat{T}_{x}=\left(\begin{array}{cccc}
\frac{B}{a^{2}} & -\frac{i A}{2 a} & -\frac{\alpha_{R}}{2 a} & 0 \\
-\frac{i A}{2 a} & -\frac{B}{a^{2}} & 0 & 0 \\
\frac{\alpha_{R}}{2 a} & 0 & \frac{B}{a^{2}} & \frac{i A}{2 a} \\
0 & 0 & \frac{i A}{2 a} & -\frac{B}{a^{2}}
\end{array}\right), \\
& \hat{T}_{y}=\left(\begin{array}{cccc}
\frac{B}{a^{2}} & \frac{A}{2 a} & \frac{i \alpha_{R}}{2 a} & 0 \\
-\frac{A}{2 a} & -\frac{B}{a^{2}} & 0 & 0 \\
\frac{i \alpha_{R}}{2 a} & 0 & \frac{B}{a^{2}} & \frac{A}{2 a} \\
0 & 0 & -\frac{A}{2 a} & -\frac{B}{a^{2}}
\end{array}\right) .
\end{aligned}
$$

Here, $\mu$ is the chemical potential and $a$ is the lattice spacing. The operators $c_{i, j}^{\dagger}$ and $c_{i, j}$ create and annihilate an electron in the lattice site $(i, j)$.

The ferromagnetic leads are similarly modelled by $\mathcal{H}_{B H Z}^{t b}$ with $A=0$ and $\alpha_{R}=0$ in order to decouple $\left|E_{1}, m_{j}= \pm \frac{1}{2}\right\rangle$ and $\left|H_{1}, m_{j}= \pm \frac{3}{2}\right\rangle$ bands. The ferromagnetic properties of the leads are captured by including a Zeeman splitting term $\hat{V}_{Z}[104]$,

$$
\hat{V}_{Z}=H_{0}\left(\begin{array}{cccc}
0 & 0 & e^{-i \theta} & 0 \\
0 & 0 & 0 & 0 \\
e^{i \theta} & 0 & 0 & 0 \\
0 & 0 & 0 & 0
\end{array}\right)
$$


5 Spin Field-Effect Transistor in a Quantum Spin-Hall Device

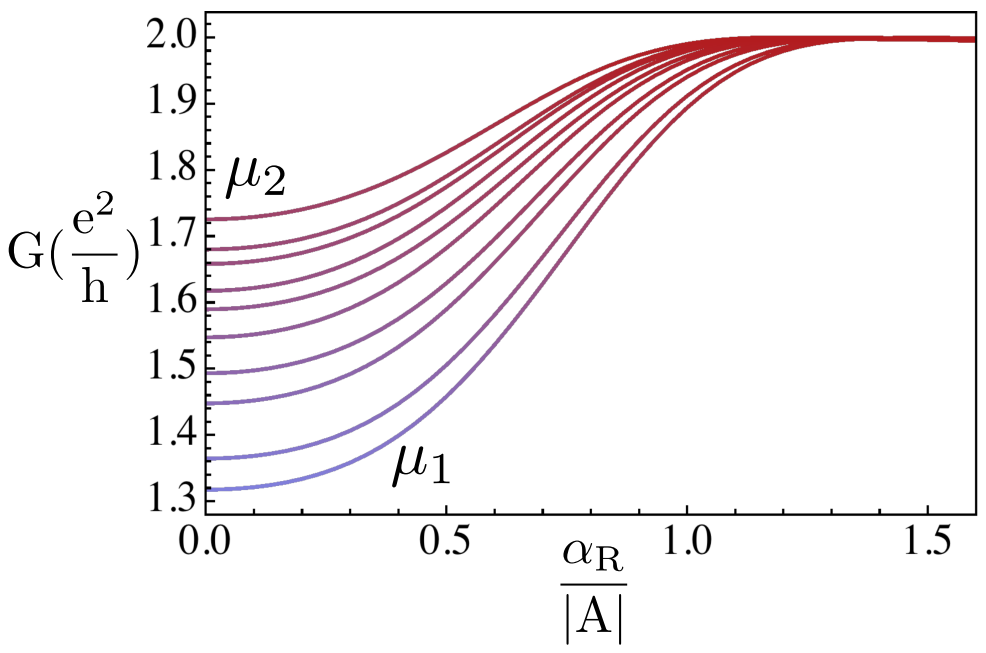

Figure 5.6: Rashba modulated conductance when $\theta=\pi$ in the right lead. In order to inject spin polarized electrons we consider an in-plane magnetic field $H_{0}$. The plot shows different modulations of the conductance when varying the chemical potential $\left(\mu \in\left[\mu_{1}, \mu_{2}\right]\right)$ in the leads. All energies are normalized to $\left|A^{2} / B\right|$.

where $H_{0}$ is the strength of the magnetic field and $\theta$ is the relative in-plane magnetization angle between the two ferromagnetic leads. The magnetic field only couples to the $\left|E_{1}, m_{j}= \pm \frac{1}{2}\right\rangle$ bands, and hence we can tune $H_{0}$ and the chemical potential in the leads in such a way to only occupy a majority of $\left|E_{1}, m_{j}=+\frac{1}{2}\right\rangle$ bands. In this way, even if both spin polarized carriers are injected, we produce effectively a spin polarized current.

The conductance of the system has been calculated by using the Kwant code [111]. In Fig. 5.6 we plot the conductance as a function of the Rashba strength, when the two ferromagnetic leads have opposite magnetizations. For chemical potentials $\mu \sim \mu_{1}$, the occupied energy bands contain mostly one type of spin polarized carriers. This causes a larger suppression of the conductance for small values of $\alpha_{R}$, in agreement with the modulation found in Section 5.2.2. For sufficiently large couplings the conductance is again $\frac{2 e^{2}}{h}$ signalling that the incoming electronic spin is being reversed. The spintilting of the edge states studied in Section 5.1 reflects directly onto the 
transport properties of the system. As expected, we observe the same enhancement of the conductance as the one found along the cut at $\theta=\pi$ in the density plot in Fig. 5.5. Indeed this numerical modulation of the conductance matches perfectly with the results obtained in Sections 5.2.2 and 5.2.3.

\subsection{Conclusions}

In this work we have shown how a QSH insulator with Rashba SOC can be used to modulate an electric current and manipulate the spin of injected electrons. The setup we propose allows for an all-electric control of the outgoing spin current making it an interesting possibility for a spin fieldeffect transistor. It is important to stress that system geometry and size are not particularly relevant as long as the two paths are symmetric and shorter than the phase relaxation length. Moreover, since the QSH edge states are topologically protected, transport is not affected by (weak) nonmagnetic disorder. Electrons will always fully transmit across the QSH and the conductance will only depend on the scattering at the interface between the QSH disk and the leads. 



\section{Tuning topology in thin films of topological insulators by strain gradients}

In condensed matter systems characterized by low-energy Dirac or Weyl quasiparticles, the coupling between electronic and lattice degrees of freedom leads to remarkable effects. In graphene [112], for instance, elastic deformations of the lattice due to strains couple to the Dirac fermions as a pseudomagnetic vector potential $[43,113,114]$. Spatially inhomogeneous strains then typically result in a pseudomagnetic field, which, in its simplest form, gives rise to a pseudo-Landau level spectrum at low energies [43]. Such strain-induced pseudo-Landau levels have been observed using scanning tunneling microscopy techniques both in graphene [115] and in artificial graphene [116]. When the pseudomagnetic fields are spatially nonuniform and periodically modulated [117], electron-electron interactions can trigger an integer quantum Hall effect without any external time-reversal-breaking field and thus similar in nature to the Haldane phase [118].

In three-dimensional topological semimetals with Weyl quasiparticles [70] instead, pseudomagnetic fields due to inhomogeneous strains [119] have been predicted to yield an enhancement of the bulk conductivity due to an underlying chiral pseudomagnetic effect [120]. Moreover, time-dependent mechanical deformations due to, e.g., acoustic waves create a related pseudoelectric field [121]. The concomitant presence of pseudoelectric and pseudomagnetic fields can also lead to a pseudo-Landau level collapse [122], which generalizes the Landau level collapse that occurs when perpendicular ordinary electric and magnetic fields are present.

Low-energy Dirac quasiparticles are also naturally realized at the boundaries of time-reversal invariant strong three-dimensional topological insulators (3DTIs) [24, 123]. Contrary to the Dirac states of graphene, however, these surface states are anomalous: on each separate surface they violate the fermion doubling theorem [124] and thus form half an ordinary twodimensional (semi)metal. This apparent paradox is resolved by considering that the Dirac states appearing at the opposite surface cancel the anomaly 
6 Tuning topology in thin films of topological insulators by strain gradients

and hence regularize the system.

A 3DTI thin film then can be thought of as forming a graphene ana$\log$ but where the Dirac quasiparticles are separated in real space rather than momentum space, and acquire a thickness-dependent mass due to the symmetry-allowed tunneling effect between opposite surfaces. Moreover, these geometries represent the ideal playground where inhomogeneous strain effects become pervasive. Thin films are much more flexible than bulk crystals and can bend easily $[125,126]$. In conventional semiconducting systems, substantial bending strain effects have been both theoretically predicted [127] and experimentally observed [128]. Additionally, in thin films grown on a substrate a lattice mismatch at the interface yields a strain that gradually relaxes away from the interface thus providing yet another source of non uniform strains.

Starting out from these observations, in this chapter we show that already in their simplest form inhomogeneous strains couple to the Dirac fermions of 3DTI thin films in an entirely different manner as compared to graphene and topological semimetals. Bending strains trigger indeed a phase transition between two topologically distinct insulating phases. In materials with large bulk band gaps, strain gradients result in a structure inversion asymmetry term driving a topological phase transition from a quantum spin-Hall (QSH) phase to a conventional band insulator [129]. Remarkably, we find that in materials with strongly reduced bulk band gaps inhomogeneous strains have an opposite effect, and promote a topologically non-trivial QSH phase thanks to a strain-gradient analog of the quantum-confined Stark effect [130].

\subsection{Strained TI thin films}

In order to analyze the influence of inhomogeneous strains in thin films of strong 3DTIs, we start out by introducing the effective bulk $\mathbf{k} \cdot \mathbf{p}$ Hamiltonian close to the $\Gamma$ point of the Brillouin zone (BZ) for the $\mathrm{Bi}_{2} \mathrm{Se}_{3}$ family of materials. It can be derived using the theory of invariants which accounts for the essential point group symmetries of the $R \overline{3} m$ (No. 166) space group, namely the inversion symmetry $\mathcal{I}$ and the three-fold rotation symmetry along the $z$-axis $\mathcal{C}_{3 z}$, with the addition of time-reversal symmetry $\mathcal{T}$. Using as basis states the $\Gamma$-point spin-orbit coupled parity eigenstates $\left|P 1_{z}^{+}, \frac{1}{2}\right\rangle,\left|P 2_{z}^{-}, \frac{1}{2}\right\rangle,\left|P 1_{z}^{+},-\frac{1}{2}\right\rangle,\left|P 2_{z}^{-},-\frac{1}{2}\right\rangle$, which are derived from the hy- 
bridized $p_{z}$ orbitals of $\mathrm{Bi}$ and $\mathrm{Se}$, the three symmetries can be represented as $\mathcal{T}=i\left(\sigma_{2} \otimes \tau_{0}\right) \mathcal{K}, \mathcal{I}=\sigma_{0} \otimes \tau_{3}$ and $\mathcal{C}_{3 z}=\exp \left(i(\pi / 3) \sigma_{3} \otimes \tau_{0}\right)$, with $\mathcal{K}$ being the complex conjugation, and $\sigma$ and $\tau$ the Pauli matrices acting in the spin and orbital space, respectively. With this, the low-energy continuum bulk Hamiltonian [131] can be cast in the following form

$$
\begin{aligned}
\mathcal{H}_{3 \mathrm{DTI}}(\mathbf{k})=\epsilon_{0}(\mathbf{k}) \sigma_{0} \otimes & \tau_{0}+\left(-M+B_{1} k_{z}^{2}+B_{2} k_{\|}^{2}\right) \sigma_{0} \otimes \tau_{3} \\
& +A_{1} k_{z} \sigma_{3} \otimes \tau_{1}+A_{2} k_{x} \sigma_{1} \otimes \tau_{1}+A_{2} k_{y} \sigma_{2} \otimes \tau_{1},
\end{aligned}
$$

where $\epsilon_{0}(\mathbf{k})=D_{1} k_{z}^{2}+D_{2} k_{\|}^{2}$ and $k_{\|}^{2}=k_{x}^{2}+k_{y}^{2}$. In addition, $A_{1,2}, B_{1,2}, D_{1,2}$ and $M$ are material-dependent parameters. In the Hamiltonian above we have neglected, without loss of generality, the momentum independent term proportional to the identity $\sigma_{0} \otimes \tau_{0}$ since it corresponds to a rigid shift of all energies. In the $M B_{1,2}>0$ inverted band regime, this model predicts the appearance of an idealized single surface Dirac cones thus verifying the $\mathbb{Z}_{2}=1$ non-trivial value of the strong topological index [132]. Since the bulk band gap $M$ in the stoichiometric materials is much larger than the energy scale of room temperature, external perturbations such as strain or pressure are expected to change the topology of the system only above a critical threshold: for $\mathrm{Bi}_{2} \mathrm{Se}_{3}$ it has been predicted that a topological phase transition from a strong 3D TI to a normal band insulator $[133,134]$ occurs at a critical uniaxial strain $\simeq 7 \%$. This is different from the case of, for instance, $\mathrm{HgTe}$ where a change from tensile to compressive strain drives a topological phase transition from a strong 3DTI to a Weyl semimetal phase [135].

However, the situation can be drastically different in a thin film where the surface Dirac cones located at opposite surfaces start to hybridize with each other. This hybridization, in fact, yields a surface energy gap $\delta m \propto M e^{-w / l_{s}}$ where $w$ is the thin film thickness, while $l_{s}$ is the decay length of the topologically protected surface states [136]. Whenever the surface energy gap is larger than a (low-temperature) thermal energy, the thin film then realizes a two-dimensional time-reversal invariant insulating state, which can be characterized by the so-called Fu-Kane-Mele $\mathbb{Z}_{2}$ topological invariant [20, 137]. Furthermore, since the band gap is narrow, strain effects can and do lead to a change in the topology of the system. To prove the assertion above, we explicitly include strain effects in the effective bulk continuum Hamiltonian Eq. 6.1. Making use of the usual Bir-Pikus scheme [138], and neglecting 
6 Tuning topology in thin films of topological insulators by strain gradients

the possible presence of shear strains, the Hamiltonian for a generic biaxial strain takes the following form

$$
\begin{aligned}
\mathcal{H}_{\text {strain }}=\left[C_{1} \epsilon_{z z}+C_{2}\left(\epsilon_{x x}+\epsilon_{y y}\right)\right] & \sigma_{0} \otimes \tau_{0}+ \\
& +\left[M_{1} \epsilon_{z z}+M_{2}\left(\epsilon_{x x}+\epsilon_{y y}\right)\right] \sigma_{0} \otimes \tau_{3},
\end{aligned}
$$

where $C_{1,2}$ and $M_{1,2}$ are the deformation potentials of the material at hand.

Although generally breaking the three-fold rotation symmetry $\mathcal{C}_{3 v}$, homogeneous strains have a negligible effect even in thin film structures. This is immediately apparent considering the fact that besides a trivial rigid shift of the energy, a generic homogeneous strain merely renormalizes the bulk energy gap $M$ and consequently the thin film surface band gap $\delta m$ without affecting the topology of the system. However, and this is key, this does not hold true for inhomogeneous strains.

Let us consider for instance the strain pattern due to a mechanical bending of the thin film. Such a mechanical deformation has been very recently achieved by placing single-crystalline $\mathrm{Bi}_{2} \mathrm{Se}_{3}$ nanowires over deep trenches [139]. It can be also realized by external force loading using an atomic force microscope as recently employed to induce large flexoelectric effects in strontium titanate single crystals [140]. As schematically shown in Fig. 6.1, in a mechanically bent thin film the top (bottom) surface is dilated while the bottom (top) surface is compressed. This, in turn, implies a structural inversion asymmetry that, as we show below, is decisive in tailoring the topological properties of the 3DTI thin film.

\subsection{Strain gradient-induced topological phase transitions}

To simplify our treatment, we will consider in the remainder an idealized bending with the local curvatures of the mechanical neutral plane [see Supplemental Material] that are constant. This produces a strain pattern $\epsilon_{x x}=$ $\epsilon_{y y}=-\alpha z$ with $\alpha$ constant and $z$ measured from the mechanical neutral plane, whereas $\epsilon_{z z}=-\nu \epsilon_{x x} /(1-\nu)$ with $\nu$ the Poisson ratio. Hence, the strain Hamiltonian Eq. 6.2 simply becomes

$$
\mathcal{H}_{\text {strain }}(z)=u_{0} \frac{z}{w} \sigma_{0} \otimes \tau_{0}+u_{3} \frac{z}{w} \sigma_{0} \otimes \tau_{3},
$$




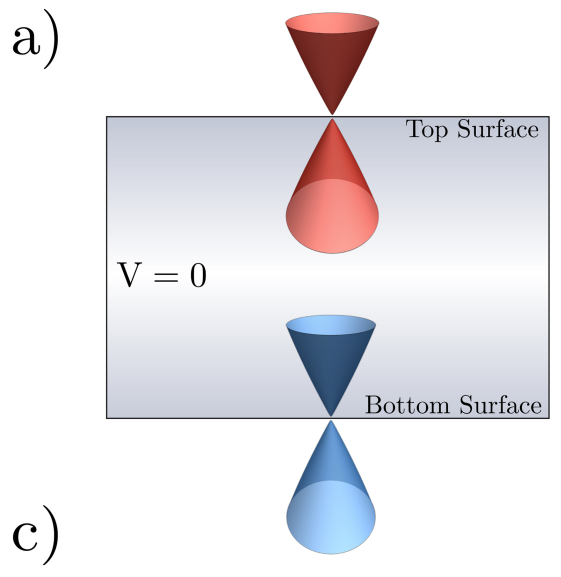

b)
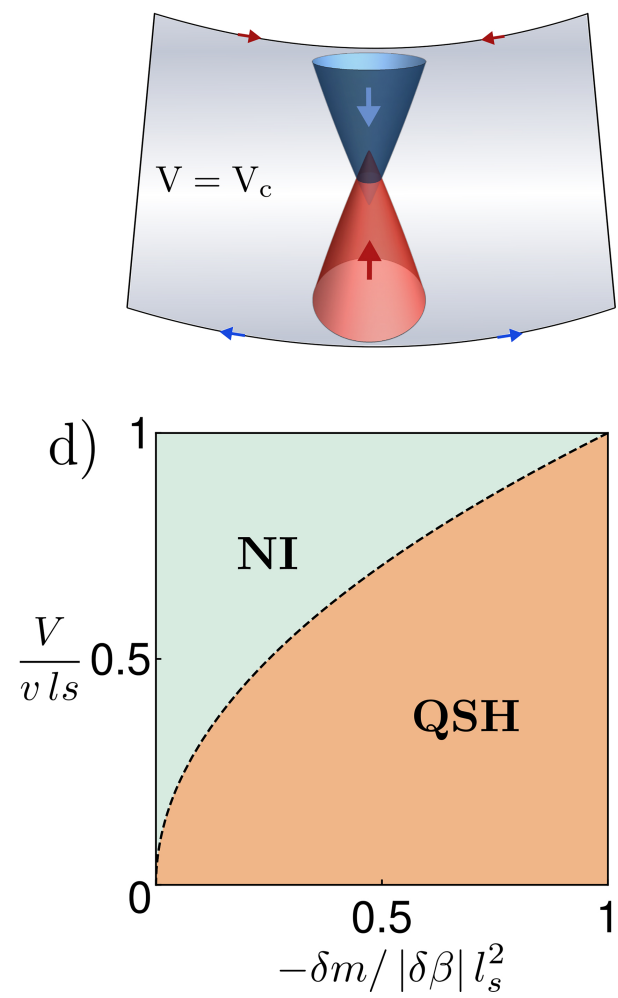

Figure 6.1: (color online) Sketch of the surface band structure of a bent 3DTI thin film. a) In the strain-free configuration the top and bottom surface Dirac cones acquire an hybridization gap at the Dirac point. b) At a critical bending radius the two gapped Dirac cones are pulled toward each other and touch on a nodal line $|\mathbf{k}|=k_{c}$. c) For even larger bending radii the surface band gap is reopened with the system that then corresponds to a conventional band insulator. d) Corresponding topological phase diagram in terms of the strain-gradient induced structure inversion parameter $V$ and the ratio between the hybridization surface gap $\delta m$ and surface effective mass $\delta \beta$. 
6 Tuning topology in thin films of topological insulators by strain gradients
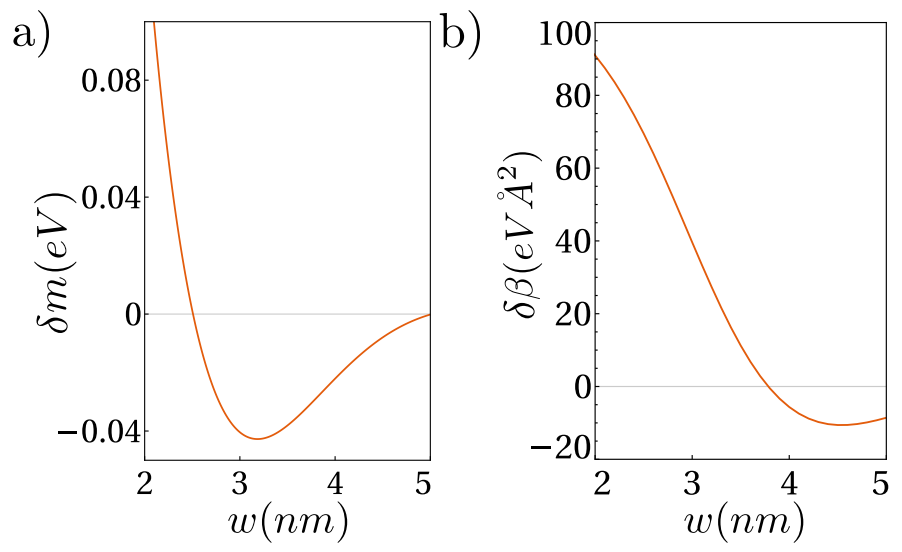

Figure 6.2: (color online) Behavior of the surface Dirac cones hybridization gap $\delta m$ (a) and the effective mass parameter $\delta \beta$ (b) as a function of the 3DTI thin film thickness $w$. We have used the $\mathbf{k} \cdot \mathbf{p}$ parameters of $\mathrm{Bi}_{2} \mathrm{Se}_{3}$ of Ref. [131]. For $2.5 \mathrm{~nm} \lesssim w \lesssim 5 \mathrm{~nm}$ the unstrained thin film realizes a QSH insulator.

where we have introduced the two parameters $u_{0,3}$ that depend on the deformation potentials, the ratio between the bending radius and the thin-film thickness, and the Poisson ratio. To analyze the effect of such inhomogeneous strain, we first solve the unstrained Hamiltonian Eq. 6.1 with open boundary conditions at the projected $\bar{\Gamma}$ point of the surface BZ. We therefore obtain two low-energy Kramers pairs $\left|E_{+}^{\uparrow \downarrow}\right\rangle$ and $\left|E_{-}^{\uparrow \downarrow}\right\rangle$ with opposite parity, whose energy $m_{ \pm}=\left\langle E_{ \pm}^{\uparrow \downarrow}\left|\mathcal{H}_{3 \mathrm{DTI}}\left(0,0,-i \partial_{z}\right)\right| E_{ \pm}^{\uparrow \downarrow}\right\rangle$ originates from the hybridization of the surface Dirac cones located at opposite surfaces. As the thin-film thickness is varied [see the Supplemental Material] these states undergo multiple crossings, which is consistent with the oscillatory crossover from 2DTI to 3DTI predicted in Ref. [141]. The inhomogeneous strain term couples equal spin states of opposite parity since, as mentioned above, it yields a structural inversion asymmetry. It thus generates a nonvanishing coupling $V=\left\langle E_{+}^{\uparrow \downarrow}\left|\mathcal{H}_{\text {strain }}(z)\right| E_{-}^{\uparrow \downarrow}\right\rangle$. This coupling, in turn, enhances the splitting of the low-energy states at the $\bar{\Gamma}$ point to $2 \sqrt{m^{2}+V^{2}}$. However, it does not preclude the possibility of band-gap closing and reopening at different points of the surface BZ. 
To account for this, we next obtain the effective $\mathbf{k} \cdot \mathbf{p}$ surface Hamiltonian in the $\left|E_{+,-}^{\uparrow \downarrow}\right\rangle$ subspace. It can be obtained by noticing that the parity eigenstates at the $\bar{\Gamma}$ point can be written as the bonding and anti-bonding states of the two surface Dirac states appearing in semi-infinite geometries, i.e. $\left|E_{+,-}^{\uparrow \downarrow}\right\rangle=\left(\left|t^{\uparrow \downarrow}\right\rangle \pm\left|b^{\uparrow \downarrow}\right\rangle\right) / \sqrt{2}$. In the $\left|t^{\uparrow \downarrow}\right\rangle,\left|b^{\uparrow \downarrow}\right\rangle$ basis the surface Hamiltonian reads

$$
\mathcal{H}_{2 \mathrm{D}}(\mathbf{k})=\epsilon(\mathbf{k})+\left(\begin{array}{cccc}
V & -i v k_{+} & m(\mathbf{k}) & 0 \\
i v k_{-} & V & 0 & m(\mathbf{k}) \\
m(\mathbf{k}) & 0 & -V & i v k_{-} \\
0 & m(\mathbf{k}) & -i v k_{+} & -V
\end{array}\right)
$$

where $k_{ \pm}=k_{x} \pm i k_{y}$ and we introduced the momentum dependent hybridization $m(\mathbf{k})=\delta m / 2+|\delta \beta|\left(k_{x}^{2}+k_{y}^{2}\right) / 2$, with $\delta m=\left(m_{+}-m_{-}\right), \delta \beta=\left(\beta_{+}-\beta_{-}\right)$ and $\beta_{ \pm}=B_{2}\left\langle E_{ \pm}^{\uparrow \downarrow}\left|\sigma_{0} \otimes \tau_{3}\right| E_{ \pm}^{\uparrow \downarrow}\right\rangle$. The particle-hole breaking term is defined as $\epsilon(\mathbf{k})=\left(m_{+}+m_{-}\right) / 2+\left(\beta_{+}+\beta_{-}\right)\left(k_{x}^{2}+k_{y}^{2}\right) / 2$ while the Fermi velocity $v=A_{2}\left|\left\langle E_{ \pm}^{\uparrow \downarrow}\left|\sigma_{1} \otimes \tau_{1}\right| E_{\mp}^{\downarrow \uparrow}\right\rangle\right|$. For very thick films $\delta m \simeq 0$, we then recover two surface Dirac cones which, however, are pushed (pulled) to higher (lower) energies by the bending strain. This finding is perfectly compatible with low-temperature magnetotransport measurements of bent $\mathrm{Bi}_{2} \mathrm{Se}_{3}$ nanowires, which indeed show an opposite shift of the surface Dirac cones at opposite surfaces [139]. More importantly, when expressed in the original $E_{+,-}^{\uparrow \downarrow}$ basis the surface $\mathbf{k} \cdot \mathbf{p}$ Hamiltonian corresponds to the well-known BernevigHughes-Zhang (BHZ) continuum model for HgTe quantum wells [21] but with the addition of a strain-gradient induced structure-inversion-asymmetry term $\propto V$. The evolution of the band structure while continuously increasing the structure inversion asymmetry term $V$ is shown in Fig. 6.1 in the inverted band regime. For $V<V_{c}=v \sqrt{-\delta m /|\delta \beta|}$ the system is adiabatically connected to a QSH insulator with a full surface band gap. By increasing the bending strain, a topological phase transition occurs at $V=V_{c}$ with the system that for $V>V_{c}$ becomes a conventional band insulator. Note that in the non-inverted regime the bending strain term $V$ does not lead to any band-gap closing and reopening points, which implies that inhomogeneous strains only act as trivializers of the system. In order to verify this physical picture, we have numerically computed [see Fig. 6.2] the hybridization gap $\delta m$ and the effective mass parameters $\delta \beta$ for thin films of varying thickness 
6 Tuning topology in thin films of topological insulators by strain gradients

$w$ using the $\mathbf{k} \cdot \mathbf{p}$ parameters for $\mathrm{Bi}_{2} \mathrm{Se}_{3}$ as obtained from density functional theory calculations [131]. Using that $v \simeq A_{2} \simeq 4 \mathrm{eV} A$, we find that thin films of $2.6 \mathrm{~nm}$ thickness can be driven via the topological phase transition for a strain-gradient induced coupling of $V \simeq 55 \mathrm{meV}$. By considering that strain-induced shift of Dirac cones of $V \simeq 30 \mathrm{meV}$ have been achieved with a maximal strain at the top (bottom) surface of $\simeq \pm 0.1 \%$ [139], one can therefore expect that the strain-gradient induced topological phase transition would require a maximal strain $\ll 1 \%$, or equivalently a bending radius of $\gg 260 \mathrm{~nm}$. A critical bending radius of the same order of magnitude can be expected for $\mathrm{Sb}_{2} \mathrm{Te}_{3}$ thin films [see the Supplemental Material for the behavior of the hybridization gap $\delta m$ and $\delta \beta$ for this material]. Signature of this topological phase transition can be then obtained by performing longitudinal resistance measurements similar to those performed in HgTe quantum wells [22]. For bending radii larger than the critical one, the topological nature of the QSH state would result in a residual conductance of $2 e^{2} / h$ when the chemical potential is in the thin-film gap. Increasing bending beyond the critical radius, instead, the residual conductance should be exponentially small precisely as in a conventional band insulator.

The tunability of the topology in 3DTI thin films by means of inhomogeneous strains is similar in nature to the one achievable by means of externally applied electric fields, since they also provide a source of structure inversion asymmetry. It is thus natural to expect that when considering Cr-doped and $\mathrm{V}$-doped $\mathrm{Bi}_{2} \mathrm{Te}_{3}$ thin films - in the inverted band regime they gain considerable spin susceptibility through the van Vleck paramagnetism thereby developing long-range magnetic order with a quantized anomalous Hall effect [142] - mechanical deformations will yield an analog of the Stark effect induced magnetic quantum phase transition theoretically predicted [143] and experimentally verified [144] in these materials.

\subsection{Narrow band-gap materials}

Having established the occurrence of strain-gradient induced topological phase transitions in materials where there is a large separation between the surface energy gap $\delta m$ and the bulk gap $M$, we next turn our attention to strong 3DTI that in their bulk are at the verge of a topological phase transition to a normal band insulator. In the $\mathrm{Bi}_{2} \mathrm{Se}_{3}$ material class, a substantial 


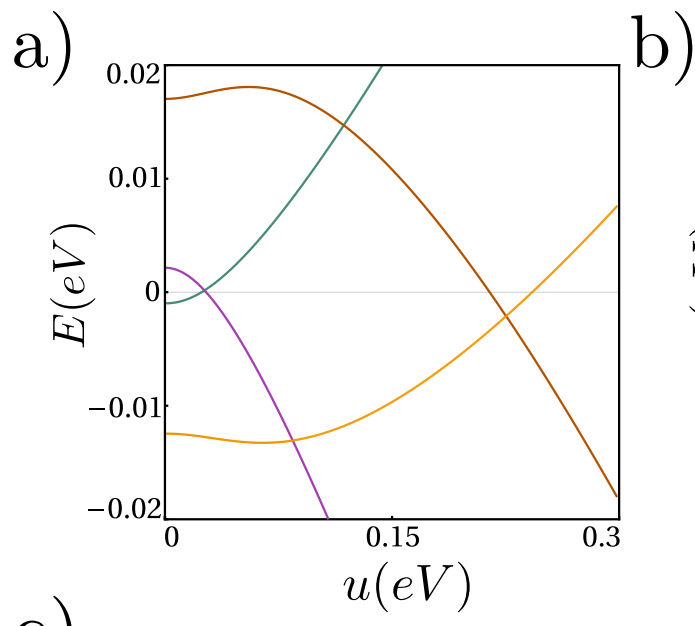

b)

c)
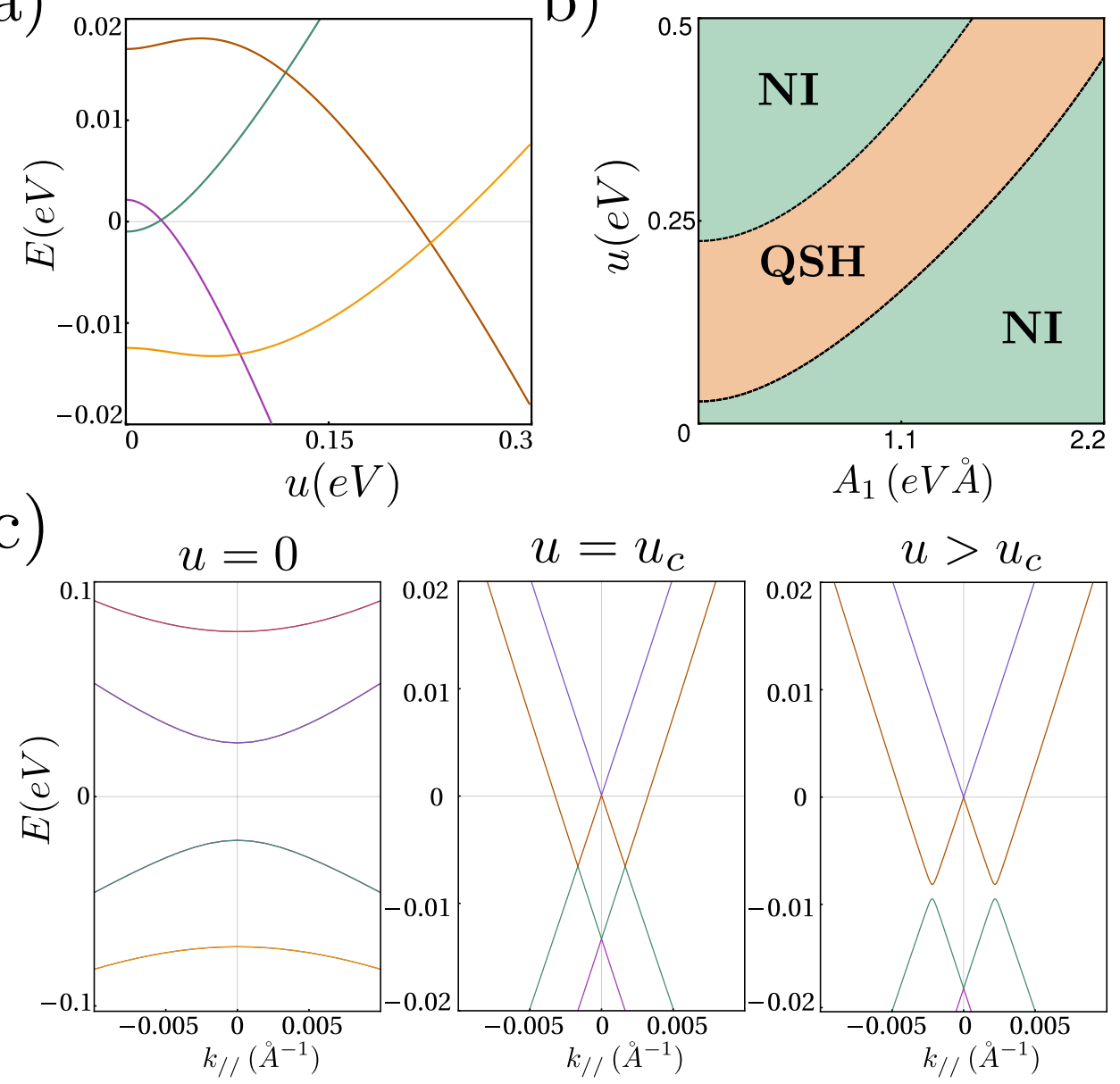

Figure 6.3: (color online) a) Evolution of the $\bar{\Gamma}$ point energy levels for a narrow band gap 3DTI thin film with $A_{1}=0$ as the strain coupling term $u=u_{0}$ is increased. The ratio between the strain coupling terms has been fixed to $u_{0} / u_{3}=0.2$. (b) Topological phase diagram in the $A_{1}$ vs strain plane. (c) Evolution of the band structure across the topological phase transition for $A_{1}=2.2 \mathrm{ev} \stackrel{\circ}{A}$. 
6 Tuning topology in thin films of topological insulators by strain gradients

decrease in the bulk band gap can be obtained [145] by increasing the Se content in Cr-doped $\mathrm{Bi}_{2}\left(\mathrm{Se}_{x} \mathrm{Te}_{1-x}\right)_{3}$. Similarly, density functional theory calculations [146] predict a topological phase transition in $\mathrm{Sb}_{2}\left(\mathrm{Te}_{1-x} \mathrm{Se}_{x}\right)_{3}$. Thin film structures of these alloys realize conventional band insulators due to a strong hybridization of the surface Dirac cones, in much the same way as ultra-thin films of $\mathrm{Bi}_{2} \mathrm{Se}_{3}$ but with thicknesses that can reach the tens of nanometers scale. In this case, however, the transversal subbands are very close to each other, which hence requires to go beyond the simple lowenergy picture used so far. We have therefore numerically diagonalized the full Hamiltonian $\mathcal{H}_{3 \text { DTI }}(\mathbf{k})+\mathcal{H}_{\text {strain }}(z)$ for a $15 \mathrm{~nm}$ thick film considering a substantial decrease of the bulk gap to $M=2.8 \mathrm{meV}$ while starting from the special case $A_{1} \equiv 0$. Fig. 6.3(a) shows the evolution of the $\bar{\Gamma}$ point spin-degenerate subbands while increasing the strain couplings terms. The electron $\left|E_{n}\right\rangle$ and hole $\left|H_{n}\right\rangle$ subbands are not coupled by the strain gradients terms. Moreover, in each of the sectors the strain gradients realize an analog of the quantum-confined Stark effect [130] and thus push the electron (hole) levels downwards (upwards). Hence, at a critical strain coupling $u_{c}$ there is a crossing between the $\left|E_{1}\right\rangle$ and $\left|H_{1}\right\rangle$ subbands, which corresponds to a topological phase transition to a QSH phase. In fact, by projecting the full Hamiltonian onto these low-energy states, one finds that the effective Hamiltonian corresponds to the BHZ model with preserved two-dimensional $\mathbf{k}_{\|} \rightarrow-\mathbf{k}_{\|}$inversion symmetry.

Next we consider the effect of turning on the $A_{1}$ term. Since the crossing between $\left|E_{n}\right\rangle$ and $\left|H_{n}\right\rangle$ at the $\bar{\Gamma}$ are not protected by parity, they are changed to anticrossings for finite $A_{1}$ values [see the Supplemental Material]. However, by invoking the principle of adiabatic continuity the full band structure must still exhibit bandgap closing and reopening points which coincide with topological phase transitions. The ensuing topological phase diagram is shown in Fig. 6.3(b). Note that the band-gap closing reopening points occurs in this case on a nodal line $\mathbf{k}_{\|}=k_{c}$ as can be seen from the evolution of the band structure in Fig. 6.3(c). We finally note that this strain-gradient induced phase transition from normal insulator to QSH insulators can be also assisted via the application of an external electric field analogously to the electrical-induced topological transitions predicted to occur in few-layer phosphorene [147]. 


\subsection{Conclusions}

To sum up, we have shown that bending strains couple to the massive Dirac quasiparticles of 3DTI thin films in such a way to trigger phase transitions between topologically distinct insulating phases. Bending strains have been already proposed as a mean to control the spin transport properties of quantum spin Hall insulators [148]. We have shown that in thin films, instead, they can change the topology of the system itself. In stoichiometric materials with large bulk band gaps, the strain-induced structure inversion asymmetry generically endangers the parent QSH phase thus driving a topological phase transition to a normal band insulator. In alloys with small bulk band gaps instead bending strains have a completely opposite effect, and can promote a topologically non-trivial phase as a result of the existence of a strain gradient analog of the quantum-confined Stark effect. The strain-gradient induced topological phase transition discussed in this work can also trigger magnetic quantum phase transitions in magnetic topological insulator thin films $[143,144]$. This strain gradient tunability of magnetism could be employed in proposals for strain-assisted transistor devices as well as magnetic random access memories. 
6 Tuning topology in thin films of topological insulators by strain gradients

\section{A Appendix}

\section{A.1 Derivation of the inhomogeneous strain $\mathrm{k} \cdot \mathrm{p}$ Hamiltonian}

We first derive the strain pattern due to a mechanical bending of the 3DTI thin film and recall that the strain tensor $\overline{\bar{u}}$ determines how an element of length $d l$ changes after the system is deformed. It is well known that for small deformations the components of $u$ can be expressed as,

$$
u_{i j}=\frac{1}{2}\left(\frac{\partial u_{i}}{\partial u_{j}}+\frac{\partial u_{j}}{\partial u_{i}}\right)
$$

where $u_{i}$ is the displacement of a point in space due to the deformation. When a plate is bent, one surface of the system is compressed while the opposite is dilated. In the middle of the plate there exists a neutral surface that is neither compressed nor dilated and in the following we will take our coordinate system to be centered on said neutral surface. By considering the thickness of the plate to be smaller than the deformations along the two other directions, we can write the components of the strain tensor along the three principal axes in the form:

$$
\left\{\begin{array}{l}
u_{x x}=-z \frac{\partial^{2} \zeta}{\partial x^{2}} \\
u_{y y}=-z \frac{\partial^{2} \zeta}{\partial y^{2}} \\
u_{z z}=z \frac{\sigma}{1-\sigma}\left(\frac{\partial^{2} \zeta}{\partial x^{2}}+\frac{\partial^{2} \zeta}{\partial y^{2}}\right)
\end{array}\right.
$$

In the above definitions we have introduced the vertical displacement $\zeta(x, y)$ (see Fig. 6.4) of a point on the neutral surface and denoted with $\sigma \in(0,1 / 2)$ the Poisson ratio. We consider the deformation to be isotropic along the two in plane directions $x$ and $y$, hence $\partial^{2} \zeta / \partial x^{2}=\partial^{2} \zeta / \partial y^{2}$. For the sake of simplicity we define the quantities,

$$
\begin{aligned}
& u_{0}=C_{1} w \frac{\sigma}{1-\sigma}\left(\frac{\partial^{2} \zeta}{\partial x^{2}}+\frac{\partial^{2} \zeta}{\partial y^{2}}\right)-C_{2} w\left(\frac{\partial^{2} \zeta}{\partial x^{2}}+\frac{\partial^{2} \zeta}{\partial y^{2}}\right) \\
& u_{3}=M_{1} w \frac{\sigma}{1-\sigma}\left(\frac{\partial^{2} \zeta}{\partial x^{2}}+\frac{\partial^{2} \zeta}{\partial y^{2}}\right)-M_{2} w\left(\frac{\partial^{2} \zeta}{\partial x^{2}}+\frac{\partial^{2} \zeta}{\partial y^{2}}\right)
\end{aligned}
$$

Assuming a constant bending radius, i.e. $\partial^{2} \zeta / \partial x^{2}=\partial^{2} \zeta / \partial y^{2}=$ const., the quantities defined above and the expressions for the strain components, the 
6.A Appendix

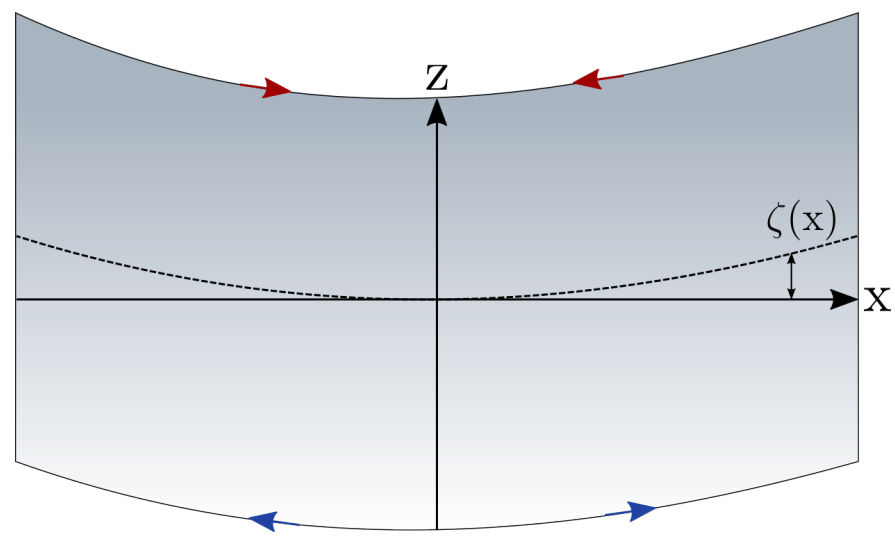

Figure 6.4: Vertcal displacement $\zeta(x)$ of a point on a neutral line of the bent plate.

strain $\mathbf{k} \cdot \mathbf{p}$ Hamiltonian reported in Eq. 6.2 of the main text can be written in the following form:

$$
H_{\text {strain }}(z)=u_{0} \frac{z}{w} \sigma_{0} \otimes \tau_{0}+u_{3} \frac{z}{w} \sigma_{0} \otimes \tau_{3}
$$


6 Tuning topology in thin films of topological insulators by strain gradients

\section{A.2 $\mathrm{Sb}_{2} \mathrm{Te}_{3}$ Thin films}
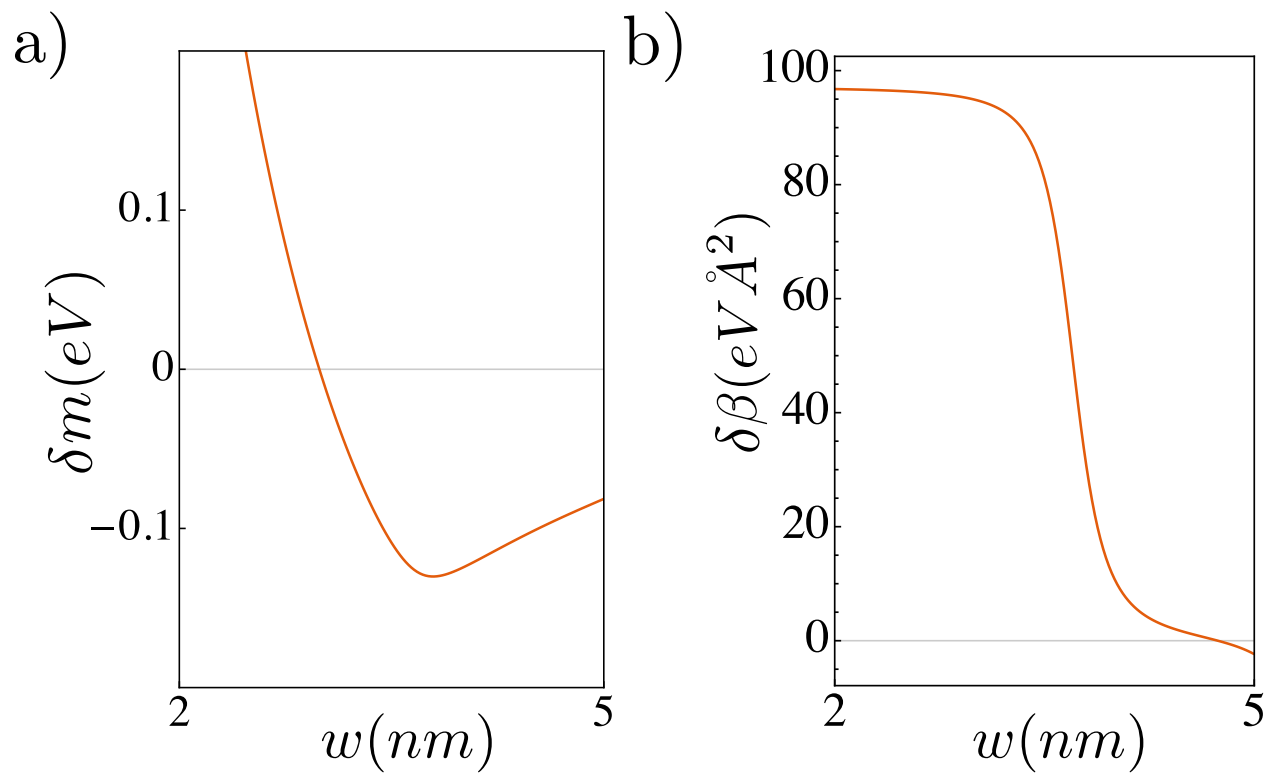

Figure 6.5: Behavior of the surface Dirac cones hybridization gap $\delta m$ (a) and the effective mass parameter $\delta \beta$ (b) as a function of the 3DTI thin film thickness $w$. We have used the $\mathbf{k} \cdot \mathbf{p}$ parameters of $\mathrm{Sb}_{2} \mathrm{Te}_{3}$ of Ref. [136]. For $2.5 \mathrm{~nm} \lesssim w \lesssim 5 \mathrm{~nm}$ the unstrained thin film realizes a QSH insulator. 


\section{A.3 Supplemental Figures}

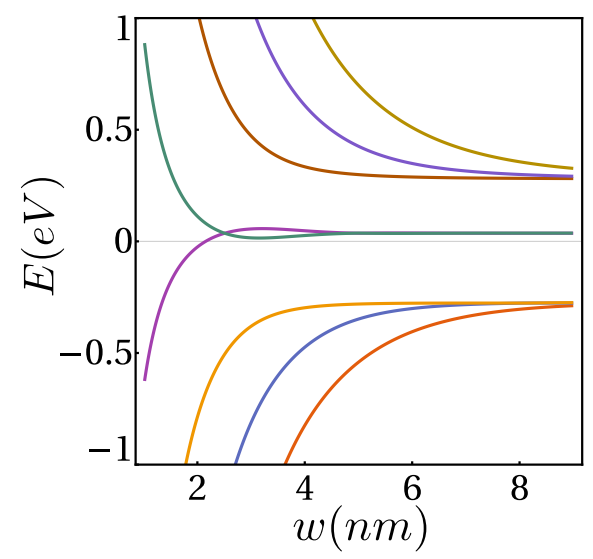

Figure 6.6: The evolution of the $\bar{\Gamma}$ energy levels of a $\mathrm{Bi}_{2} \mathrm{Se}_{3}$ thin films as the thickness is varied. The crossing between the low-energy levels correspond to topological phase transitions.

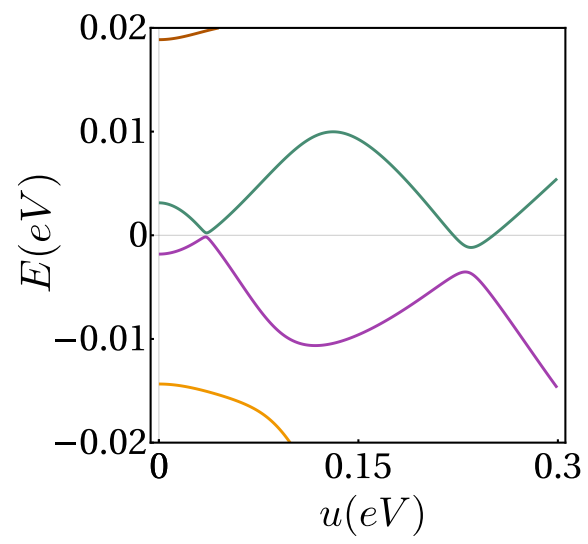

Figure 6.7: Evolution of the $\bar{\Gamma}$ energy levels for a narrow band gap TI thin film with thickness $w=15 \mathrm{~nm}$ and $A_{1} \neq 0$. The crossings encountered at $A_{1}=0$ [see Fig. 3 of the main text] are changed into anticrossings. 



\section{Conclusions}

This thesis has two main themes. The first one, discussed in Chapters 2-4, is the study of various Berry curvature effects present in two-dimensional systems. We have first shown how strained bilayer graphene hosts a finite Berry curvature dipole and linked its presence to the warping of the Fermi surface. In the following chapter we uncovered a Berry curvature-induced anomalous planar Hall effect appearing in trigonal crystals with spin-orbit coupling. This effect is expected to be present not only in crystals with a honeycomb structure hosting Dirac cones, but also in materials with low energy theories described by a Rashba 2DEG.

The second main theme is based on the study of the peculiar properties of two and three-dimensional topological insulators (Chapters 5,6). We have shown that, by tuning the Rashba coupling, a quantum spin-Hall disk acts as an all electric spin-transistor when coupled symmetrically to two ballistic leads. We then moved on to discuss how strain gradients can alter the topology of three-dimensional topological insulators leading to topological phase transitions.

\subsection{Summary and Results}

In Chapter 2 we have shown how the non-linear Hall effect can arise in time-reversal symmetric materials without spin-orbit coupling or tilted Dirac cones. We have found that by applying uniaxial strain to monolayer and bilayer graphene a non-vanishing Berry curvature dipole appears. The warping of the Fermi surface is the triggering mechanism needed to explain such effect.

In Chapter 3 we have unveiled the presence of an anomalous type of planar Hall effect (PHE) in two-dimensional trigonal crystals. The PHE is the appearance of an in-plane transverse voltage in the presence of coplanar electric and magnetic fields. Its hallmark is a characteristic $\pi$-periodic, i.e. even under a magnetic field reversal, angular dependence with the trans- 


\section{Conclusions}

verse voltage that exactly vanishes when the electric and magnetic fields are aligned. Here we have demonstrated that in two-dimensional trigonal crystals Zeeman-induced non-trivial Berry curvature effects yield a previously unknown anomalous PHE that is odd in the magnetic field and independent of the relative angle with the driving electric field. Moreover, we have shown that when an additional mirror symmetry forces the transverse voltage to vanish in the linear response regime, the anomalous PHE can occur as a second-order response at both zero and twice the frequency of the applied electric field. This non-linear PHE possesses an antisymmetric quantum contribution that originates from a Zeeman-induced Berry curvature dipole.

In Chapter 4 we have studied the presence of the anomalous PHE in a Rashba 2DEG by employing an effective $2 \times 2$ model, typically used to describe the low energy properties of materials around the high symmetry point $\Gamma$. We have then found that when including higher order warping terms with threefold rotational symmetry, the Berry curvature of the model becomes non-trivial and the anomalous PHE appears.

In Chapter 5 we have discussed the transport properties of a quantum spinHall insulator with sizeable Rashba spin-orbit coupling in a disk geometry. We have found that the presence of topologically protected helical edge states allows for the control and manipulation of spin polarized currents: when ferromagnetic leads are coupled to the quantum spin-Hall device, the ballistic conductance is modulated by the Rashba strength. We have then showed that, by tuning the Rashba interaction via an all-electric gating, it is possible to control the spin polarization of injected electrons.

In Chapter 6 we have theoretically shown that the coupling of inhomogeneous strains to the Dirac fermions of three-dimensional topological insulators (3DTI) in thin film geometries results in the occurrence of phase transitions between topologically distinct insulating phases. By means of minimal

$\mathbf{k} \cdot \mathbf{p}$ models for strong 3DTI in the $\mathrm{Bi}_{2} \mathrm{Se}_{3}$ materials class, we have found that in thin films of stoichiometric materials a strain-gradient induced structure inversion asymmetry drives a phase transition from a quantum spin-Hall phase to a topologically trivial insulating phase. Interestingly, we have discovered that in alloys with strongly reduced bulk band gaps, strain gradients have an opposite effect and promote a topologically non-trivial phase from a parent normal band insulator. These strain-gradient assisted switchings between topologically distinct phases are expected to yield a strain gradient tunability of magnetism in magnetic topological insulator thin films. 


\section{Bibliography}

[1] D. Xiao, M.-C. Chang, and Q. Niu, Rev. Mod. Phys. 82, 1959 (2010).

[2] M. Z. Hasan and C. L. Kane, Rev. Mod. Phys. 82, 3045 (2010).

[3] M. V. Berry, Proceedings of the Royal Society of London. A. Mathematical and Physical Sciences 392, 45 (1984).

[4] B. Simon, Phys. Rev. Lett. 51, 2167 (1983).

[5] V. Fock, Zeitschrift für Physik 49, 323 (1928).

[6] F. Wilczek and A. Zee, Phys. Rev. Lett. 52, 2111 (1984).

[7] D. Frustaglia and K. Richter, Phys. Rev. B 69, 235310 (2004).

[8] F. Nagasawa, D. Frustaglia, H. Saarikoski, K. Richter, and J. Nitta, Nature Communications 4, 2526 EP (2013).

[9] Y. A. Bychkov and E. I. Rashba, Journal of Physics C: Solid State Physics 17, 6039 (1984).

[10] A. Manchon, H. C. Koo, J. Nitta, S. M. Frolov, and R. A. Duine, Nature Materials 14, 871 (2015).

[11] F. E. Meijer, A. F. Morpurgo, and T. M. Klapwijk, Phys. Rev. B 66, 033107 (2002).

[12] C. Ortix, Spin interference effects in rashba quantum rings, in Physics of Quantum Rings, edited by V. M. Fomin (Springer International Publishing, Cham, 2018) pp. 327-346.

[13] S. Datta, Electronic Transport in Mesoscopic Systems, Cambridge Studies in Semiconductor Physics and Microelectronic Engineering (Cambridge University Press, 1995). 
Bibliography

[14] J. Zak, Phys. Rev. Lett. 62, 2747 (1989).

[15] F. D. M. Haldane, Phys. Rev. Lett. 93, 206602 (2004).

[16] H. Weng, R. Yu, X. Hu, X. Dai, and Z. Fang, Advances in Physics 64, 227 (2015), https://doi.org/10.1080/00018732.2015.1068524 .

[17] D. J. Thouless, M. Kohmoto, M. P. Nightingale, and M. den Nijs, Phys. Rev. Lett. 49, 405 (1982).

[18] I. Sodemann and L. Fu, Phys. Rev. Lett. 115, 216806 (2015).

[19] K. v. Klitzing, G. Dorda, and M. Pepper, Phys. Rev. Lett. 45, 494 (1980).

[20] C. L. Kane and E. J. Mele, Phys. Rev. Lett. 95, 146802 (2005).

[21] B. A. Bernevig, T. L. Hughes, and S.-C. Zhang, Science 314, 1757 (2006), http://science.sciencemag.org/content/314/5806/1757.full.pdf

[22] M. König, S. Wiedmann, C. Brüne, A. Roth, H. Buhmann, L. W. Molenkamp, X.-L. Qi, and S.-C. Zhang, Science 318, 766 (2007), http://science.sciencemag.org/content/318/5851/766.full.pdf .

[23] D. Hsieh, Y. Xia, D. Qian, L. Wray, F. Meier, J. H. Dil, J. Osterwalder, L. Patthey, A. V. Fedorov, H. Lin, A. Bansil, D. Grauer, Y. S. Hor, R. J. Cava, and M. Z. Hasan, Phys. Rev. Lett. 103, 146401 (2009).

[24] X.-L. Qi and S.-C. Zhang, Rev. Mod. Phys. 83, 1057 (2011).

[25] N. Nagaosa, J. Sinova, S. Onoda, A. H. MacDonald, and N. P. Ong, Rev. Mod. Phys. 82, 1539 (2010).

[26] Q. Niu and D. J. Thouless, Phys. Rev. B 35, 2188 (1987).

[27] J. E. Moore and J. Orenstein, Phys. Rev. Lett. 105, 026805 (2010).

[28] D. Xiao, G.-B. Liu, W. Feng, X. Xu, and W. Yao, Phys. Rev. Lett. 108, 196802 (2012).

[29] J.-S. You, S. Fang, S.-Y. Xu, E. Kaxiras, and T. Low, Phys. Rev. B 98, 121109 (2018). 
[30] Y. Zhang, J. van den Brink, C. Felser, and B. Yan, 2D Materials 5, 044001 (2018).

[31] J. Son, K.-H. Kim, Y. H. Ahn, H.-W. Lee, and J. Lee, arXiv e-prints , arXiv:1907.00010 (2019).

[32] S.-Y. Xu, Q. Ma, H. Shen, V. Fatemi, S. Wu, T.-R. Chang, G. Chang, A. M. M. Valdivia, C.-K. Chan, Q. D. Gibson, J. Zhou, Z. Liu, K. Watanabe, T. Taniguchi, H. Lin, R. J. Cava, L. Fu, N. Gedik, and P. Jarillo-Herrero, Nature Physics 14, 900 (2018).

[33] K. Kang, T. Li, E. Sohn, J. Shan, and K. F. Mak, arXiv e-prints , arXiv:1809.08744 (2018), arXiv:1809.08744 [cond-mat.mes-hall] .

[34] Z. Z. Du, C. M. Wang, H.-Z. Lu, and X. C. Xie, Phys. Rev. Lett. 121, 266601 (2018).

[35] Q. Ma, S.-Y. Xu, H. Shen, D. MacNeill, V. Fatemi, T.-R. Chang, A. M. Mier Valdivia, S. Wu, Z. Du, C.-H. Hsu, S. Fang, Q. D. Gibson, K. Watanabe, T. Taniguchi, R. J. Cava, E. Kaxiras, H.-Z. Lu, H. Lin, L. Fu, N. Gedik, and P. Jarillo-Herrero, Nature 565, 337 (2019).

[36] T. H. Hsieh, H. Lin, J. Liu, W. Duan, A. Bansil, and L. Fu, Nature Communications 3, 982 EP (2012), article.

[37] A. Lau and C. Ortix, Phys. Rev. Lett. 122, 186801 (2019).

[38] J. I. Facio, D. Efremov, K. Koepernik, J.-s. You, I. Sodemann, and J. V. D. Brink, Phys. Rev. Lett. 121, 246403 (2018).

[39] B. A. Bernevig and T. L. Hughes, Topological Insulators and Topological Superconductors (Princeton University Press, 2013).

[40] G. W. Semenoff, Phys. Rev. Lett. 53, 2449 (1984).

[41] G. Giovannetti, P. A. Khomyakov, G. Brocks, P. J. Kelly, and J. van den Brink, Phys. Rev. B 76, 073103 (2007).

[42] C. R. Woods, L. Britnell, A. Eckmann, R. S. Ma, J. C. Lu, H. M. Guo, X. Lin, G. L. Yu, Y. Cao, R. . V. Gorbachev, A. V. Kretinin, J. Park, L. A. Ponomarenko, M. I. Katsnelson, Y. N. Gornostyrev, 
Bibliography

K. Watanabe, T. Taniguchi, C. Casiraghi, H.-J. Gao, A. K. Geim, and K. . S. Novoselov, Nature Physics 10, 451 EP (2014).

[43] F. Guinea, M. I. Katsnelson, and A. K. Geim, Nature Physics 6, 30 (2009).

[44] F. de Juan, M. Sturla, and M. A. H. Vozmediano, Phys. Rev. Lett. 108, 227205 (2012).

[45] E. McCann and M. Koshino, Reports on Progress in Physics 76, 056503 (2013).

[46] J. B. Oostinga, H. B. Heersche, X. Liu, A. F. Morpurgo, and L. M. Vandersypen, Nature Materials 7, 151 (2008).

[47] J. R. Schrieffer and P. A. Wolff, Physical Review 149, 149 (1966).

[48] E. McCann and V. I. Fal'ko, Phys. Rev. Lett. 96, 086805 (2006).

[49] M. Mucha-Kruczynski, E. McCann, and V. I. Fal'ko, Semiconductor Science and Technology 25, 033001 (2010).

[50] M. Mucha-Kruczyński, I. L. Aleiner, and V. I. Fal'ko, Physical Review B - Condensed Matter and Materials Physics 84, 041404(R) (2011).

[51] T. Ohta, A. Bostwick, T. Seyller, K. Horn, and E. Rotenberg, Science 313, 951 (2006).

[52] K. S. Novoselov, A. K. Geim, S. V. Morozov, D. Jiang, Y. Zhang, S. V. Dubonos, I. V. Grigorieva, and A. A. Firsov, Science 306, 666 (2004).

[53] H. X. Tang, R. K. Kawakami, D. D. Awschalom, and M. L. Roukes, Phys. Rev. Lett. 90, 107201 (2003).

[54] M. Bowen, K.-J. Friedland, J. Herfort, H.-P. Schönherr, and K. H. Ploog, Phys. Rev. B 71, 172401 (2005).

[55] Z. Ge, W. L. Lim, S. Shen, Y. Y. Zhou, X. Liu, J. K. Furdyna, and M. Dobrowolska, Phys. Rev. B 75, 014407 (2007).

[56] N. Wadehra, R. Tomar, R. M. Varma, R. K. Gopal, Y. Singh, S. Dattagupta, and S. Chakraverty, Nature Communications 11, 874 (2020). 
[57] A. Joshua, J. Ruhman, S. Pecker, E. Altman, and S. Ilani, Proceedings of the National Academy of Sciences 110, 9633 (2013), https://www.pnas.org/content/110/24/9633.full.pdf .

[58] G. Yin, J.-X. Yu, Y. Liu, R. K. Lake, J. Zang, and K. L. Wang, Phys. Rev. Lett. 122, 106602 (2019).

[59] A. A. Burkov, Phys. Rev. B 96, 041110 (2017).

[60] S. Nandy, G. Sharma, A. Taraphder, and S. Tewari, Phys. Rev. Lett. 119, 176804 (2017).

[61] F. C. Chen, X. Luo, J. Yan, Y. Sun, H. Y. Lv, W. J. Lu, C. Y. Xi, P. Tong, Z. G. Sheng, X. B. Zhu, W. H. Song, and Y. P. Sun, Phys. Rev. B 98, 041114 (2018).

[62] Z. Wang, Y. Sun, X.-Q. Chen, C. Franchini, G. Xu, H. Weng, X. Dai, and Z. Fang, Phys. Rev. B 85, 195320 (2012).

[63] Z. K. Liu, B. Zhou, Y. Zhang, Z. J. Wang, H. M. Weng, D. Prabhakaran, S.-K. Mo, Z. X. Shen, Z. Fang, X. Dai, Z. Hussain, and Y. L. Chen, Science 343, 864 (2014).

[64] S.-M. Huang, S.-Y. Xu, I. Belopolski, C.-C. Lee, G. Chang, B. Wang, N. Alidoust, G. Bian, M. Neupane, C. Zhang, S. Jia, A. Bansil, H. Lin, and M. Z. Hasan, Nature Communications 6, 7373 (2015).

[65] H. Weng, C. Fang, Z. Fang, B. A. Bernevig, and X. Dai, Phys. Rev. X 5, 011029 (2015).

[66] B. Q. Lv, H. M. Weng, B. B. Fu, X. P. Wang, H. Miao, J. Ma, P. Richard, X. C. Huang, L. X. Zhao, G. F. Chen, Z. Fang, X. Dai, T. Qian, and H. Ding, Phys. Rev. X 5, 031013 (2015).

[67] B. Q. Lv, N. Xu, H. M. Weng, J. Z. Ma, P. Richard, X. C. Huang, L. X. Zhao, G. F. Chen, C. E. Matt, F. Bisti, V. N. Strocov, J. Mesot, Z. Fang, X. Dai, T. Qian, M. Shi, and H. Ding, Nature Physics 11, 724 (2015).

[68] S.-Y. Xu, I. Belopolski, N. Alidoust, M. Neupane, G. Bian, C. Zhang, R. Sankar, G. Chang, Z. Yuan, C.-C. Lee, S.-M. Huang, H. Zheng, 
Bibliography

J. Ma, D. S. Sanchez, B. Wang, A. Bansil, F. Chou, P. P. Shibayev, H. Lin, S. Jia, and M. Z. Hasan, Science 349, 613 (2015).

[69] S.-Y. Xu, N. Alidoust, I. Belopolski, Z. Yuan, G. Bian, T.-R. Chang, H. Zheng, V. N. Strocov, D. S. Sanchez, G. Chang, C. Zhang, D. Mou, Y. Wu, L. Huang, C.-C. Lee, S.-M. Huang, B. Wang, A. Bansil, H.-T. Jeng, T. Neupert, A. Kaminski, H. Lin, S. Jia, and M. Zahid Hasan, Nature Physics 11, 748 (2015).

[70] N. P. Armitage, E. J. Mele, and A. Vishwanath, Rev. Mod. Phys. 90, 015001 (2018).

[71] L. P. He, X. C. Hong, J. K. Dong, J. Pan, Z. Zhang, J. Zhang, and S. Y. Li, Phys. Rev. Lett. 113, 246402 (2014).

[72] T. Liang, Q. Gibson, M. N. Ali, M. Liu, R. J. Cava, and N. P. Ong, Nature Materials 14, 280 (2015).

[73] C.-L. Zhang, S.-Y. Xu, I. Belopolski, Z. Yuan, Z. Lin, B. Tong, G. Bian, N. Alidoust, C.-C. Lee, S.-M. Huang, T.-R. Chang, G. Chang, C.-H. Hsu, H.-T. Jeng, M. Neupane, D. S. Sanchez, H. Zheng, J. Wang, H. Lin, C. Zhang, H.-Z. Lu, S.-Q. Shen, T. Neupert, M. Zahid Hasan, and S. Jia, Nature Communications 7, 10735 (2016).

[74] Q. Li, D. E. Kharzeev, C. Zhang, Y. Huang, I. Pletikosić, A. V. Fedorov, R. D. Zhong, J. A. Schneeloch, G. D. Gu, and T. Valla, Nature Physics 12, 550 (2016).

[75] J. Xiong, S. K. Kushwaha, T. Liang, J. W. Krizan, M. Hirschberger, W. Wang, R. J. Cava, and N. P. Ong, Science 350, 413 (2015).

[76] M. Hirschberger, S. Kushwaha, Z. Wang, Q. Gibson, S. Liang, C. A. Belvin, B. A. Bernevig, R. J. Cava, and N. P. Ong, Nature Materials 15, 1161 (2016).

[77] A. A. Taskin, H. F. Legg, F. Yang, S. Sasaki, Y. Kanai, K. Matsumoto, A. Rosch, and Y. Ando, Nature Communications 8, 1340 (2017).

[78] P. He, S. S.-L. Zhang, D. Zhu, S. Shi, O. G. Heinonen, G. Vignale, and H. Yang, Phys. Rev. Lett. 123, 016801 (2019). 
[79] R. Battilomo, N. Scopigno, and C. Ortix, Phys. Rev. Lett. 123, 196403 (2019).

[80] D. Wawrzik, J.-S. You, J. I. Facio, J. van den Brink, and I. Sodemann, The infinite berry curvature of weyl fermi arcs (2020), arXiv:2010.10537 [cond-mat.mes-hall] .

[81] A. Kormányos, G. Burkard, M. Gmitra, J. Fabian, V. Zólyomi, N. D. Drummond, and V. Fal'ko, 2D Materials 2, 022001 (2015).

[82] S. Manzeli, D. Ovchinnikov, D. Pasquier, O. V. Yazyev, and A. Kis, Nature Reviews Materials 2, 17033 (2017).

[83] R. Karplus and J. M. Luttinger, Phys. Rev. 95, 1154 (1954).

[84] S. Nandy and I. Sodemann, Phys. Rev. B 100, 195117 (2019).

[85] Y. Gao, S. A. Yang, and Q. Niu, Phys. Rev. Lett. 112, 166601 (2014).

[86] C. L. Kane and E. J. Mele, Phys. Rev. Lett. 95, 226801 (2005).

[87] D. Huertas-Hernando, F. Guinea, and A. Brataas, Phys. Rev. B 74, 155426 (2006).

[88] T. Fukui, Y. Hatsugai, and H. Suzuki, Journal of the Physical Society of Japan $\mathbf{7 4}, 1674$ (2005).

[89] G. D. Mahan, Many-Particle Physics, 2nd ed. (Plenum, New York, N.Y., 1993).

[90] L. Fu, Phys. Rev. Lett. 103, 266801 (2009).

[91] I. Žutić, J. Fabian, and S. Das Sarma, Rev. Mod. Phys. 76, 323 (2004).

[92] S. Datta and B. Das, Applied Physics Letters 56, 665 (1990), https://doi.org/10.1063/1.102730 .

[93] J. Nitta, F. E. Meijer, and H. Takayanagi, Applied Physics Letters 75, 695 (1999), https://doi.org/10.1063/1.124485 .

[94] B. Molnár, F. M. Peeters, and P. Vasilopoulos, Phys. Rev. B 69, 155335 (2004). 


\section{Bibliography}

[95] M. König, A. Tschetschetkin, E. M. Hankiewicz, J. Sinova, V. Hock, V. Daumer, M. Schäfer, C. R. Becker, H. Buhmann, and L. W. Molenkamp, Phys. Rev. Lett. 96, 076804 (2006).

[96] H. Saarikoski, A. A. Reynoso, J. P. Baltanás, D. Frustaglia, and J. Nitta, Phys. Rev. B 97, 125423 (2018).

[97] Z.-J. Ying, P. Gentile, C. Ortix, and M. Cuoco, Phys. Rev. B 94, 081406 (2016).

[98] Y. Aharonov and D. Bohm, Phys. Rev. 115, 485 (1959).

[99] Y. Aharonov and A. Casher, Phys. Rev. Lett. 53, 319 (1984).

[100] F. Nagasawa, J. Takagi, Y. Kunihashi, M. Kohda, and J. Nitta, Phys. Rev. Lett. 108, 086801 (2012).

[101] W. Chen, W.-Y. Deng, J.-M. Hou, D. N. Shi, L. Sheng, and D. Y. Xing, Phys. Rev. Lett. 117, 076802 (2016).

[102] C. Liu, T. L. Hughes, X.-L. Qi, K. Wang, and S.-C. Zhang, Phys. Rev. Lett. 100, 236601 (2008).

[103] J. Maciejko, E.-A. Kim, and X.-L. Qi, Phys. Rev. B 82, 195409 (2010).

[104] D. G. Rothe, R. W. Reinthaler, C.-X. Liu, L. W. Molenkamp, S.-C. Zhang, and E. M. Hankiewicz, New Journal of Physics 12, 065012 (2010).

[105] P. Michetti and P. Recher, Phys. Rev. B 83, 125420 (2011).

[106] L. Ortiz, R. A. Molina, G. Platero, and A. M. Lunde, Phys. Rev. B 93, 205431 (2016).

[107] A. Rod, T. L. Schmidt, and S. Rachel, Phys. Rev. B 91, 245112 (2015).

[108] R. Winkler, Spin-orbit coupling effects in two-dimensional electron and hole systems, Springer tracts in modern physics (Springer, Berlin, 2003).

[109] F. Zhang, C. L. Kane, and E. J. Mele, Phys. Rev. B 86, 081303 (2012). 
[110] C. Ortix, Phys. Rev. B 91, 245412 (2015).

[111] C. W. Groth, M. Wimmer, A. R. Akhmerov, and X. Waintal, New Journal of Physics 16, 063065 (2014).

[112] A. H. Castro Neto, F. Guinea, N. M. R. Peres, K. S. Novoselov, and A. K. Geim, Rev. Mod. Phys. 81, 109 (2009).

[113] M. Vozmediano, M. Katsnelson, and F. Guinea, Physics Reports 496, 109 (2010).

[114] B. Amorim, A. Cortijo, F. de Juan, A. Grushin, F. Guinea, A. Gutierrez-Rubio, H. Ochoa, V. Parente, R. Roldan, P. San-Jose, J. Schiefele, M. Sturla, and M. Vozmediano, Physics Reports 617, 1 (2016).

[115] N. Levy, S. A. Burke, K. L. Meaker, M. Panlasigui, A. Zettl, F. Guinea, A. H. C. Neto, and M. F. Crommie, Science 329, 544 (2010).

[116] K. K. Gomes, W. Mar, W. Ko, F. Guinea, and H. C. Manoharan, Nature 483, 306 (2012).

[117] J. W. F. Venderbos and L. Fu, Phys. Rev. B 93, 195126 (2016).

[118] F. D. M. Haldane, Phys. Rev. Lett. 61, 2015 (1988).

[119] A. Cortijo, Y. Ferreirós, K. Landsteiner, and M. A. H. Vozmediano, Phys. Rev. Lett. 115, 177202 (2015).

[120] A. G. Grushin, J. W. F. Venderbos, A. Vishwanath, and R. Ilan, Phys. Rev. X 6, 041046 (2016).

[121] D. I. Pikulin, A. Chen, and M. Franz, Phys. Rev. X 6, 041021 (2016).

[122] V. Arjona, E. V. Castro, and M. A. H. Vozmediano, Phys. Rev. B 96, 081110 (2017).

[123] M. Z. Hasan and C. L. Kane, Rev. Mod. Phys. 82, 3045 (2010).

[124] H. Nielsen and M. Ninomiya, Physics Letters B 130, 389 (1983).

[125] O. G. Schmidt and K. Eberl, Nature 410, 168 (2001). 
Bibliography

[126] H. Peng, W. Dang, J. Cao, Y. Chen, D. Wu, W. Zheng, H. Li, Z.-X. Shen, and Z. Liu, Nature Chemistry 4, 281 (2012).

[127] C. Ortix, S. Kiravittaya, O. G. Schmidt, and J. van den Brink, Phys. Rev. B 84, 045438 (2011).

[128] Y. Mei, S. Kiravittaya, M. Benyoucef, D. J. Thurmer, T. Zander, C. Deneke, F. Cavallo, A. Rastelli, and O. G. Schmidt, Nano Letters 7, 1676 (2007).

[129] W.-Y. Shan, H.-Z. Lu, and S.-Q. Shen, New Journal of Physics 12, 043048 (2010).

[130] P. Cendula, S. Kiravittaya, and O. G. Schmidt, Journal of Applied Physics 111, 043105 (2012).

[131] S.-C. Zhang, X. Dai, X.-L. Qi, C.-X. Liu, H. Zhang, and Z. Fang, Nat. Phys. 5, 438 (2009).

[132] L. Fu and C. L. Kane, Physical Review B 76, 045302 (2007).

[133] W. Liu, X. Peng, C. Tang, L. Sun, K. Zhang, and J. Zhong, Physical Review B 84, 245105 (2011).

[134] S. M. Young, S. Chowdhury, E. J. Walter, E. J. Mele, C. L. Kane, and A. M. Rappe, Physical Review B 84, 085106 (2011).

[135] J. Ruan, S.-K. Jian, H. Yao, H. Zhang, S.-C. Zhang, and D. Xing, Nature Communications 7, 11136 EP (2016).

[136] C. X. Liu, X. L. Qi, H. Zhang, X. Dai, Z. Fang, and S. C. Zhang, Phys. Rev. B 82, 1 (2010).

[137] L. Fu and C. L. Kane, Phys. Rev. B 74, 195312 (2006).

[138] M. R. Brems, J. Paaske, A. M. Lunde, and M. Willatzen, New Journal of Physics 20, 053041 (2018).

[139] C. Schindler, C. Wiegand, J. Sichau, L. Tiemann, K. Nielsch, R. Zierold, and R. H. Blick, Applied Physics Letters 111, 171601 (2017). 
[140] M.-M. Yang, D. J. Kim, and M. Alexe, Science 360, 904 (2018).

[141] C. X. Liu, H. Zhang, B. Yan, X. L. Qi, T. Frauenheim, X. Dai, Z. Fang, and S. C. Zhang, Physical Review B(R) 81, 041307 (2010).

[142] R. Yu, W. Zhang, H.-J. Zhang, S.-C. Zhang, X. Dai, and Z. Fang, Science 329, 61 (2010).

[143] J. Wang, B. Lian, and S. C. Zhang, Physical Review Letters 115, 036805 (2015).

[144] Z. Zhang, X. Feng, J. Wang, B. Lian, J. Zhang, C. Chang, M. Guo, Y. Ou, Y. Feng, S.-C. Zhang, K. He, X. Ma, Q.-K. Xue, and Y. Wang, Nature Nanotechnology 12, 953 EP (2017).

[145] J. Zhang, C.-Z. Chang, P. Tang, Z. Zhang, X. Feng, K. Li, L.-l. Wang, X. Chen, C. Liu, W. Duan, K. He, Q.-K. Xue, X. Ma, and Y. Wang, Science 339, 1582 (2013).

[146] W. Zhang, R. Yu, H.-J. Zhang, X. Dai, and Z. Fang, New Journal of Physics 12, 065013 (2010).

[147] Q. Liu, X. Zhang, L. B. Abdalla, A. Fazzio, and A. Zunger, Nano Letters 15, 1222 (2015).

[148] B. Huang, K.-H. Jin, B. Cui, F. Zhai, J. Mei, and F. Liu, Nature Communications 8, 15850 (2017). 



\section{English Summary}

Topology is the branch of mathematics that studies how the properties of an object are preserved under 'smooth' deformations. In a topological sense a donut and a coffee mug are very much similar, since the two have the same number of holes, they can be transformed into one another by applying some some 'smooth' transformation. In the same way a sphere is not topologically equivalent to any of the previous examples: deforming a sphere into a donut would require us to tear a hole into it, a deformation which is by no means smooth. It turns out that the number of holes of an object is a topological invariant, a discrete number that can only change by some integer quantity (there are is no such thing as a fractional hole). Objects that are classified with the same invariant are said to be topologically equivalent.

However, this thesis is about condensed matter physics, with a specific focus on the electronic properties of crystals. This naturally leads to the question: how does topology, an abstract field of mathematics, have anything to do with condensed matter physics, a field of physics that typically is concerned with the physical properties of matter?

In 1980 the physicist von Klitzing discovered that by placing an electron gas under a strong perpendicular magnetic field the Hall resistance (the resistance that develops in the direction perpendicular to the applied current) became quantized. Meaning that when changing the value of the applied magnetic field, provided the field was strong enough, the resistance would only change by some finite amount. This amount is known as the von Klitzing constant and it is equal to the ratio $h / e^{2}$, where $h$ is Planck's constant and $e$ is the electron's charge. This discovery sparked great interest and it was soon understood that topology was playing a role in the quantization effect.

It turns out that also insulators can be classified through different topological invariants. In this case the topological properties are encoded in the band structure and smooth deformations are all the deformations that do not close the band gap or break the symmetries of the system. In the specific case of the quantum Hall effect the topological invariant that classifies the 
system is an integer known as the Chern number. The quantization steps observed in the experiments correspond to different values of said number. What makes topological insulators extremely interesting is the fact the while the bulk of these systems is insulating, the edges are on the other hand conducting. Electrons moving on the edges move in a chiral manner and cannot backscatter with impurities. These edge states are said to be robust to external perturbations since the topology of the insulator is robust.

An essential quantity that encodes the topological properties is the Berry curvature. It can be derived from the band structure of the system and can be thought of as a magnetic field in the electron's momentum space. Its integral is equal to the Chern number when considering an insulator, whereas for metals it is responsible for an 'anomalous' type of Hall effect.

The first three chapters of the thesis are dedicated to studying how the Berry curvature determines the transport properties of two-dimensional metals. We discuss the appearance of a Berry curvature-related quantity, its dipole moment, in strained graphene systems. We also show how a previously overlooked kind of planar Hall effect can appear in trigonal crystals. This effect is also rooted in the Berry curvature of the electronic band structure.

The last two chapters investigate the peculiar properties of topological insulators. We discuss how the quantum spin Hall insulator can behave as a spin transistor and later study the possibility of strain induced topological phase transitions in three dimensional topological insulators. 


\section{Dutch Summary}

Topologie is de tak van de wiskunde die zich bezighoudt met de eigenschappen van een object die onveranderd blijven onder 'gladde' deformaties. Topologisch gezien zijn een donut en een koffiemok gelijk omdat ze beiden één gat hebben, en ze kunnen in elkaar vervormd worden door een 'gladde' transformatie. Op dezelfde wijze is een bol niet gelijk aan deze voorgaande voorbeelden: een bol transformeren naar een donut vereist dat we een gat prikken in de bol, een deformatie die we moeilijk 'glad' kunnen noemen. Het blijkt dat het aantal gaten in een object een topologische invariant is, een discreet getal dat alleen met een geheel getal kan veranderen (er is niet zoiets als een fractioneel gat). Objecten die door dezelfde invariant geclassificeerd worden zijn topologisch equivalent.

Echter, deze scriptie gaat over gecondenseerde materie natuurkunde, met een specifieke focus op de electronische eigenschappen van kristallen. Dit leidt tot de vraag: wat heeft topologie, een abstracte tak van wiskunde, te maken met gecondenseerde materie natuurkunde, een onderdeel van de natuurkunde dat zich bezighoud met de fysische eigenschappen van materialen?

In 1980 ontdekte de natuurkundige von Klitzing dat door een electrongas in een sterk loodrecht magnetisch veld te plaatsen de Hall weerstand (de weerstand loodrecht op de richting van een toegepaste stroom), gequantiseerd werd. Dit betekent dat wanneer de sterkte van het toegepaste magnetisch veld verandert, gegeven dat het veld sterk genoeg is, de weerstand alleen verandert met discrete stappen. De grootte van deze stappen staat bekend als de von Klitzing constante en is gelijk aan de ratio $h / e^{2}$, waar $h$ de Planck constante is en $e$ het elementaire ladingskwantum. Deze ontdekking wekte grote belangstelling, en men realiseerde zich al snel dat topologie een rol speelde in het quantisatie effect.

Het blijkt dat ook isolatoren geclassificeerd kunnen worden aan de hand van verschillende topologische invarianten. In dit geval zijn de topologische eigenschappen gecodeerd in de bandenstructuur, en gladde deformaties zijn deformaties die niet leiden tot het sluiten van de bandkloof of het breken 


\section{Dutch Summary}

van de symmetrieën van het systeem. In het specifieke geval van het kwantum Hall effect is de topologische invariant die het systeem classificeert een geheel getal dat bekend staat als het Chern getal. De quantisatie stappen die worden waargenomen in experimenten corresponderen met verschillende waardes van het Chern getal. Wat topologische isolatoren extreem interessant maakt is het feit dat terwijl het interieur van deze materialen isolerend is, de randen wel geleiden. Electronen op de rand van het materiaal bewegen op een chirale manier, en kunnen niet verstrooien tegen onzuiverheden in het materiaal. Deze randtoestanden zijn dus robuust onder externe perturbaties omdat de topologie van de isolator robuust is.

Een essentiële kwantiteit die topologische eigenschappen codeert, is de Berry kromming. Deze kan worden afgeleid uit de bandenstructuur van het systeem en kan worden gezien als een magnetisch veld in de impulsruimte van het elektron. De integraal van de Berry kromming is voor een isolator gelijk aan het Chern getal, terwijl het voor metalen verantwoordelijk is voor een 'anomaal' type Hall effect.

De eerste drie hoofstukken van deze scriptie zijn gewijd aan het bestuderen hoe de Berry kromming de transport eigenschappen bepaalt in tweedimensionale metalen. We bediscussiëren het verschijnen van de aan de Berry kromming geraleteerde eenheid, zijn dipool-moment, in grafeen systemen onder strain. We laten ook zien hoe een eerder over het hoofd gezien type planair Hall effect kan ontstaan in triogonale kristallen. Dit effect is ook geworteld in de Berry kromming van de elektronische bandenstructuur.

De laatste twee hoofdstukken onderzoeken de eigenaardige eigenschappen van topologische isolatoren. We bediscussiëren hoe de kwantum spin Hall isolator zich kan gedragen als een spin transistor en onderzoeken de mogelijkheid fase transities te induceren doormiddel van strain in drie-dimensionale topologische isolatoren. 


\section{Acknowledgments}

Questa tesi e il lavoro di ricerca condotto negli ultimi quattro anni non sarebbero mai stati possibili se non fosse stato per l'enorme supporto ricevuto da tutte le persone a me vicine. Il ringraziamento piu' grande va, come spesso accade, ai miei genitori, mamma Stefania e papá Diego, che sin da piccolo hanno creduto in me spingendomi a migliorare passo dopo passo. Negli anni ho imparato ad apprezzare tutto l'affetto e le attenzioni che mi avete dedicato. Una vita tranquilla con pochi ostacoli é un privilegio raro in questo mondo: so quanto avete investito in me e ve ne saró per sempre grato.

Eleonora, in questa tesi ci sei anche tu. Sia perché l'ho scritta soffrendo il freddo della tua casa veronese, sia perché in ogni momento del mio dottorato ti ho sempre avuta accanto. Quando presi la decisione di partire per l'Olanda non avevo la men che minima idea di cosa volesse dire coltivare una relazione a distanza. Dal punto di vista matematico immagino che la salute di una relazione decresca esponenzialmente sia in funzione della distanza che della durata di quest'ultima. Con impegno ed innumerevoli voli abbiamo tenuto duro e siamo riusciti a preservare il nostro amore. Abbiamo vissuto quattro anni con un piede in Italia ed uno in Olanda ma finalmente le nostre strade stanno per ricongiungersi.

Grazie a Valerio, Amedeo, Francesco, Giacomo, Alessandro, Luca (x2), Federica (x2), Alfredo, Silvia, Michela, Cristina e Camilla per la vostra amicizia da sempre. Tornare a Roma e ritrovarvi mi ricorda quali siano le mie radici e dove si trova casa.

La mia esperienza in Olanda non sarebbe stato la stessa, anzi sarebbe stata molto piú grigia, se non fosse stato per tutti gli amici che ho conosciuto in questi anni: grazie a Emanuele, Silvia, Serena, Gabriele (quello 'romano'), Gabriele (quello ligure), Irene, Elena, Gosia, Francesco, Massimiliano, Philippe, Luca, Vania e Nino.

I also want to thank all my colleagues, many of whom have become close friends, for all the time spent together and all the things you guys have tought me. Thank you Niccolo', Sander, Camilo, Guido, Enea, Erik, Tycho 
Acknowledgments

and Nick.

Grazie Carmine per avermi dato la possibilitá di intraprendere un dottorato in Olanda. É stato un vero piacere lavorare con te, ricorderó sempre questi quattro anni come un periodo felice della mia vita. 


\section{About the author}

I was born the 21st of June 1992 in Rome. Graduated from the Liceo Classico Virgilio in 2011, I decided to switch from a humanities type of education and enrolled in a Physics bachelor at the university of Rome, La Sapienza. I graduated cum laude in 2014, under the supervision of prof. Paolo Mataloni, with a thesis on quantum walks and their physical implementation with photons. In 2017 I obtained my master's degree cum laude with a thesis on the magnetotransport of the Rashba two-dimensional electron gas under the supervision of dr. Lara Benfatto and dr. Valentina Brosco. In the summer of 2017 I decided to move to Utrecht to start a PhD in condensed matter physics under the supervision of dr. Carmine Ortix. 